DESY 06-006

Cockcroft-06-05

JINST 1 P10001

\title{
Deep Inelastic Electron-Nucleon Scattering at the LHC
}

\author{
J. B. Dainton ${ }^{1}$, M. Klein ${ }^{2}$, P. Newman ${ }^{3}$, E. Perez ${ }^{4}$, F. Willeke ${ }^{2}$ \\ ${ }^{1}$ Cockcroft Institute of Accelerator Science and Technology, \\ Daresbury International Science Park, UK \\ ${ }^{2}$ DESY, Hamburg and Zeuthen, Germany \\ 3 School of Physics and Astronomy, University of Birmingham, UK \\ ${ }^{4}$ CE Saclay, DSM/DAPNIA/Spp, Gif-sur-Yvette, France
}

\begin{abstract}
The physics, and a design, of a Large Hadron Electron Collider (LHeC) are sketched. With high luminosity, $10^{33} \mathrm{~cm}^{-2} \mathrm{~s}^{-1}$, and high energy, $\sqrt{s}=$ 1.4 TeV, such a collider can be built in which a $70 \mathrm{GeV}$ electron (positron) beam in the LHC tunnel is in collision with one of the LHC hadron beams and which operates simultaneously with the LHC. The LHeC makes possible deep-inelastic lepton-hadron $(e p, e D$ and $e A)$ scattering for momentum transfers $Q^{2}$ beyond $10^{6} \mathrm{GeV}^{2}$ and for Bjorken $x$ down to the $10^{-6}$. New sensitivity to the existence of new states of matter, primarily in the lepton-quark sector and in dense partonic systems, is achieved. The precision possible with an electron-hadron experiment brings in addition crucial accuracy in the determination of hadron structure, as described in Quantum Chromodynamics, and of parton dynamics at the $\mathrm{TeV}$ energy scale. The LHeC thus complements the proton-proton and ion programmes, adds substantial new discovery potential to them, and is important for a full understanding of physics in the LHC energy range.
\end{abstract}




\section{Introduction}

Deep-inelastic lepton-hadron scattering (DIS) has long been [1, 2, 3, 4] the most accurate means of exploring the substructure of matter at short distances. Nowadays, such physics is concerned with electron-quark interactions at the highest possible energy and momentum transfer. An ep collider allows new states coupling to leptons and quarks to be produced and their quantum numbers to be determined. It is also concerned with the exploration of new forms of hadronic matter, which may be manifest in very high parton densities at very low Bjorken- $x$. With an ep collider the parton dynamics and the momentum distributions of quarks and gluons, which are crucial for the discovery and interpretation of new physics, are most accurately determined.

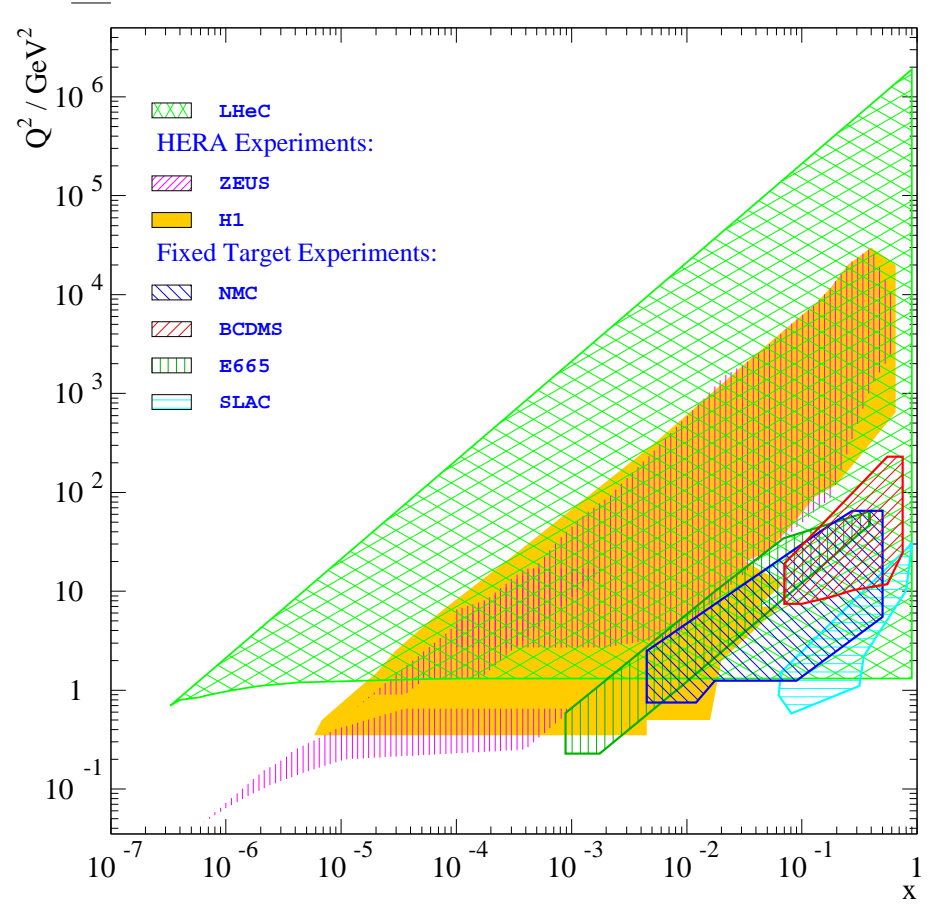

Figure 1: Kinematic regions in Bjorken-x and momentum transfers $Q^{2}$ covered by fixed target unpolarised lepton-proton scattering experiments, the $H 1$ and ZEUS experiments at HERA and the proposed electron-proton collider $\mathrm{LHeC}$.

The Large Hadron Collider (LHC) will explore a new range of energy and mass. An ep facility is essential to complete this exploration, both through its unique sensitivity to some possible new states of matter and since it provides complementary information which will be needed to resolve puzzles thrown up by $p p$ and $A A$ data. The most attractive proposition for an ep collider operating in this energy domain is to make use of the $7 \mathrm{TeV}$ LHC $p$ beam by colliding it with an intense electron or positron beam stored in a ring mounted above the LHC, a Large Hadron Electron Collider (LHeC). Compared with linac-ring solutions recently considered, such as the ILC with HERA [5] or a CLIC prototype with the LHC [6], this proposition increases the luminosity by two orders of magnitude. An $\mathrm{LHeC}$, in which for example $70 \mathrm{GeV}$ electrons collide 
with $7 \mathrm{TeV}$ protons, will substantially extend the phase space explored hitherto in deep-inelastic lepton-hadron scattering (Fig.1).

Deep-inelastic lepton-hadron physics at the $\mathrm{TeV}$ scale has been considered previously at the LEP-LHC workshop in 1990 [7, 8] and as part of the TDR for TESLA in 2001 (THERA) [9]. A ring-ring ep collider using the LHC has been considered based on LEP [10, 11, 12]. This paper is concerned with a new evaluation taking advantage of the experience gained at HERA. A feasibility study for an ep collider at the LHC using an electron ring of energy $E_{e}=70 \mathrm{GeV}$ leads to an estimated luminosity of about $10^{33} \mathrm{~cm}^{-2} \mathrm{~s}^{-1}$, which corresponds to an annual integrated luminosity of about $10 \mathrm{fb}^{-1}$, at a center of mass (cms) energy of $\sqrt{s}=2 \sqrt{E_{e} E_{p}}$ of $1.4 \mathrm{TeV}$. This places the LHeC very favourably in the luminosity-energy map of DIS facilites (Fig.2).

\section{Lepton-Proton Scattering Facilities}

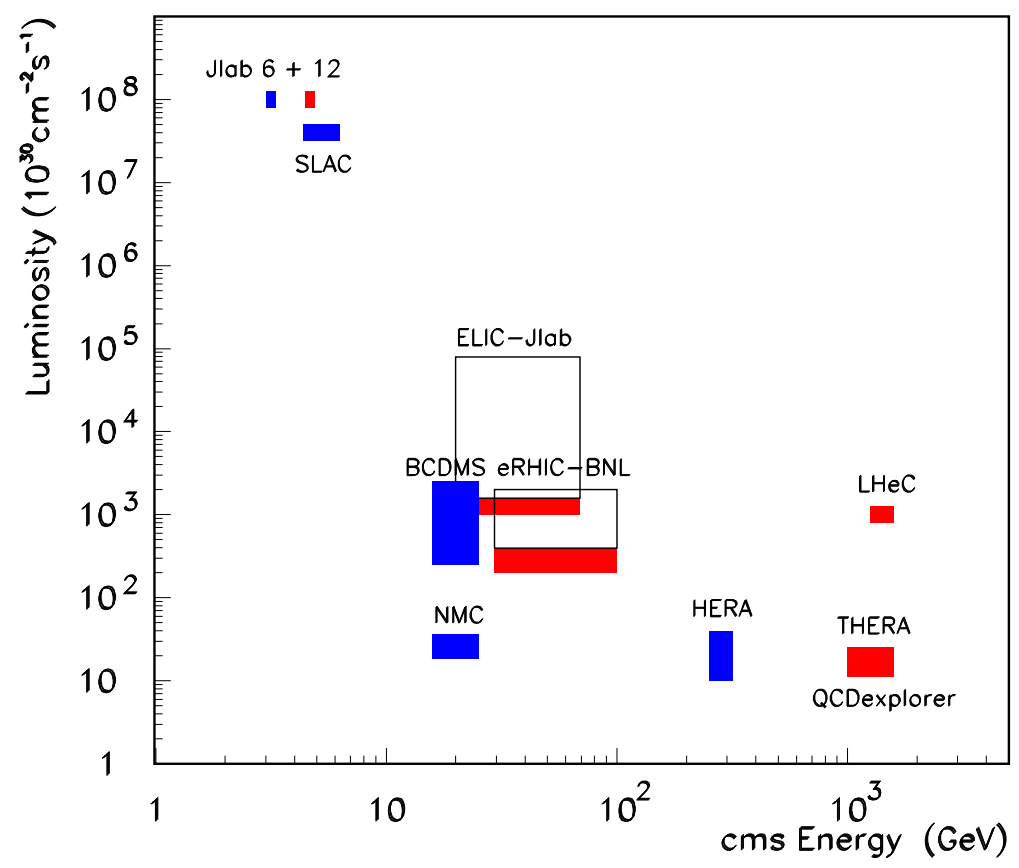

Figure 2: Summary of existing (dark, blue boxes) and proposed (grey, red boxes) facilities for unpolarised lepton-proton deep-inelastic scattering investigations in terms of the luminosity and centre-of mass energy. The Jlab fixed target programme is directed to high statistics physics at low $Q^{2}$ and very large $x$. The SLAC box indicates the luminosity of the classic ep experiment at the 2 mile linear accelerator. BCDMS and NMC have provided the most accurate DIS muon-proton structure function data using $30 \mathrm{~m}$ and $3 \mathrm{~m}$ long unpolarised hydrogen targets, respectively. The large luminosities envisaged at eRHIC and ELIC (hollow boxes), which is desirable for polarised ep physics, are based on energy-recovery linac technology. HERA has reached peak luminosities of up to $5 \cdot 10^{31} \mathrm{~cm}^{-2} \mathrm{~s}^{-1}$ with a luminosity upgrade. The linac-ring accelerator designs of THERA (TESLA/ILC-HERA) and the QCD explorer (CLIC-LHC) barely provide luminosity above $10^{31} \mathrm{~cm}^{-2} \mathrm{~s}^{-1}$. The $\mathrm{LHeC}$ is designed for the highest energy at the largest luminosity. 
This paper was first prepared for inclusion in the deliberations of the European Strategy Group of the CERN Council [13] during 2006. It highlights LHeC physics with examples and it presents a feasibility study for the machine. The scale of the LHeC is that of an upgrade of the LHC. The next steps are to complete a full evaluation of the feasibility of the LHeC, including injection, and to develop further the optimisation of an experiment and its interface to the machine. The first data at the LHC and the completion of the physics programme at HERA with its final data sample will also have a direct bearing on this optimisation.

The paper is organised as follows: In Section 2 examples are given of new physics at high masses and at very low Bjorken- $x$. Section 3 is concerned with exploiting the precision of DIS to quantify and test QCD at a new level of accuracy. Section 4 highlights the opportunities at the LHeC to probe QCD in more complex hadronic environments making use of the wide range of ion beams at the LHC. There follows a brief consideration of the kinematic reach and its implications for a detector design in Section 5. Finally, Section 6 is concerned with luminosity prospects based on an initial consideration of the LHeC machine design.

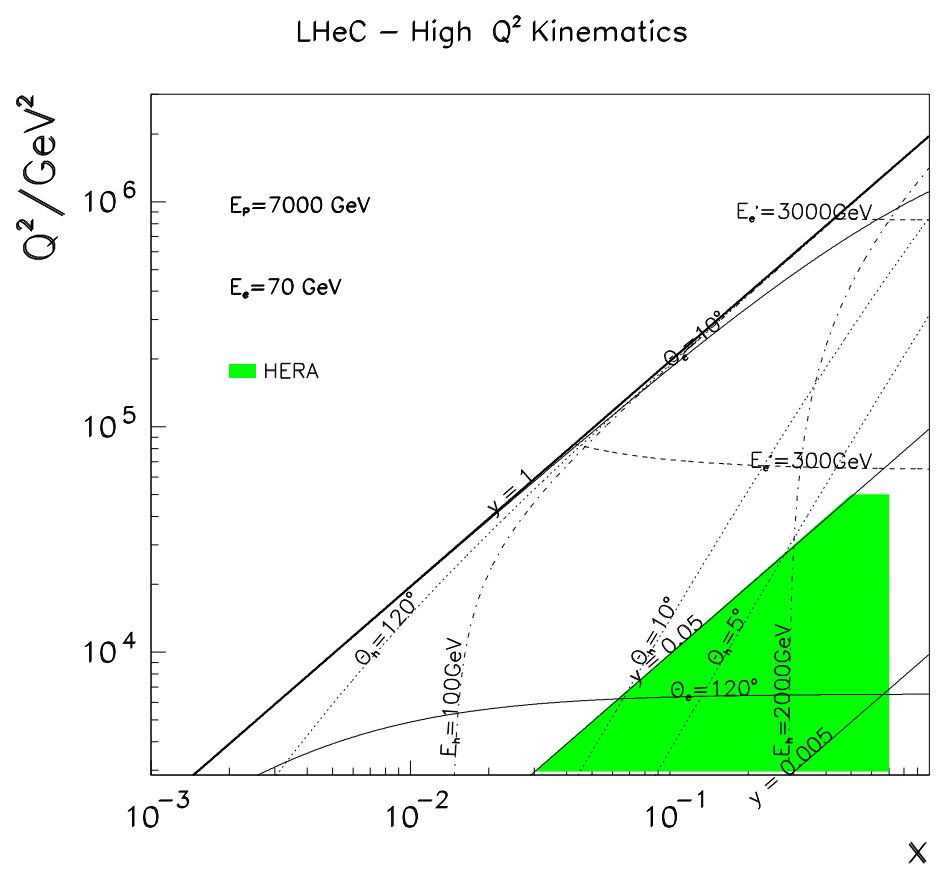

Figure 3: Kinematics of ep scattering at the LHeC at high $Q^{2}$. Solid (dotted) curves correspond to constant polar angles $\theta_{e}\left(\theta_{h}\right)$ of the scattered electron (hadronic final state). The polar angle is defined with respect to the proton beam direction. Dashed (dashed-dotted) curves correspond to constant energies $E_{e}^{\prime}\left(E_{h}\right)$ of the scattered electron (hadronic final state). The iso-angle and iso-energy lines are derived from Eq. 4, Section 5. The shaded area illustrates the region of kinematic coverage in neutral current (NC) scattering at HERA. In ep scattering electron-quark resonances can be formed with mass $M=\sqrt{x s}$. Due to luminosity and energy range, the search limit at HERA has been at about $290 \mathrm{GeV}$ while the LHeC extends to large M values of about $1300 \mathrm{GeV}$. 


\section{New Physics at the LHeC}

\subsection{Physics Beyond the Standard Model}

In the kinematic domain in which the new physics underpinning the Standard Model (SM) is manifest, new electron-quark and positron-quark dynamics could be observable, revealing a relationship between the quark and lepton sectors of the SM. This sensitivity to lepton-quark physics at the highest energy and shortest distance is at the root of the importance of the LHeC.

The high energy of the LHeC extends the kinematic range of DIS physics to much higher values of electron-quark mass $M=\sqrt{s x}$ (Fig. 3). An ep collider, providing both baryonic and leptonic quantum numbers in the initial state, is ideally suited to a study of the properties of new bosons possessing couplings to an electron-quark pair in this new mass range. Such particles can be squarks in supersymmetry with $R$-parity violation $\left(\not R_{p}\right)$, or first-generation leptoquark (LQ) bosons which appear naturally in various unifying theories beyond the Standard Model (SM). They are produced as single $s$-channel resonances via the fusion of incoming electrons with quarks in the proton. They are generically referred to as "leptoquarks" in what follows.

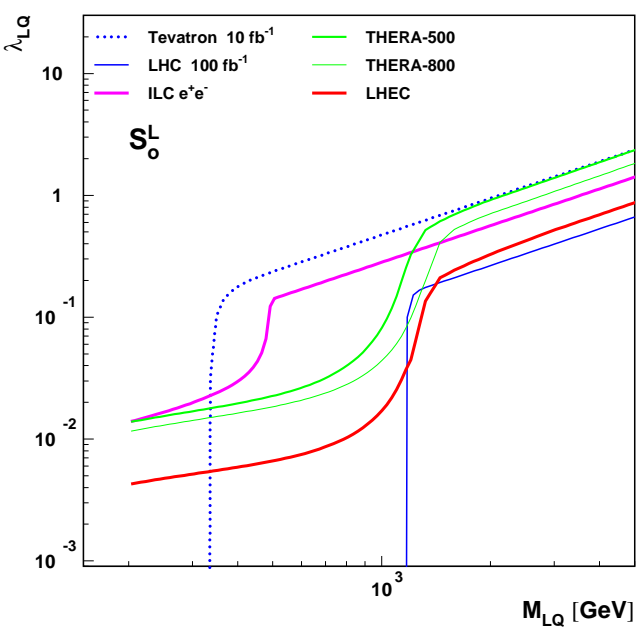

Figure 4: Mass-dependent upper bounds on the LQ coupling $\lambda$ as expected at LHeC for a luminosity of $10 \mathrm{fb}^{-1}$ (full red curve) and at the LHC for $100 \mathrm{fb}^{-1}$ (full blue curve) [14]. These are shown for an example scalar $L Q$ coupling to $e^{-} u$.

Fig. 4 shows the expected sensitivity [14] of the LHC and LHeC colliders for scalar leptoquark production. The single LQ production cross section depends on the unknown coupling $\lambda$ of the LQ to the electron-quark pair, and means for a coupling $\lambda$ of $\mathcal{O}(0.1)$, that LQ masses up to about $1 \mathrm{TeV}$ could be probed at the LHeC. In $p p$ interactions at the LHC such leptoquarks would be mainly produced via pair production or 
singly [15] with a much reduced cross section 1. In ep collisions LQ production can be probed in detail, taking advantage of the formation and decay of systems which can be observed directly as a combination of jet and lepton invariant mass in the final state. It will thereby be possible at the LHeC to probe directly and with high precision the perhaps complex structures which will result in the lepton-jet system and to determine the quantum numbers of new states. Examples of the sensitivity of high energy ep collisions to the properties of LQ production follow.

Fermion number $(F)$ : Since the parton densities for $u$ and $d$ at high $x$ are much larger than those for $\bar{u}$ and $\bar{d}$, the production cross section at LHeC of an $F=0(F=2)$ LQ is much larger in $e^{+} p\left(e^{-} p\right)$ than in $e^{-} p\left(e^{+} p\right)$ collisions. A measurement of the asymmetry between the $e^{+} p$ and $e^{-} p$ LQ cross sections thus determines the fermion number of the produced leptoquark. Pair production of first generation LQs at the LHC will not allow this determination. Single LQ production at the LHC, followed by the LQ decay into $e^{ \pm}$and $q$ or $\bar{q}$, could determine $F$ by comparing the signal cross sections with an $e^{+}$and an $e^{-}$coming from the resonant state. However, the single LQ production cross section at the LHC is two orders of magnitude lower than at the LHeC (Fig.5a), so that the asymmetry measured at the LHC may suffer from statistics in a large part of the parameter space. For a coupling $\lambda=0.1$, no information on $F$ can be extracted from the LHC data for a LQ mass above $\sim 700 \mathrm{GeV}$, while the LHeC can determine $F$ for LQ masses up to $1 \mathrm{TeV}$ (Fig.5b).

Spin: At the LHeC, the angular distribution of the LQ decay products is unambiguously related to its spin. This determination will be much more complicated, even possibly ambiguous, if only the LHC leptoquark pair production data are available. Angular distributions for vector LQs depend strongly on the structure of the $g L Q \overline{L Q}$ coupling, i.e. on possible anomalous couplings. For a structure similar to that of the $\gamma W W$ vertex, vector LQs produced via $q \bar{q}$ fusion are unpolarised and, because both LQs are produced with the same helicity, the distribution of the LQ production angle will be similar to that of a scalar LQ. The study of LQ spin via single LQ production at the LHC will suffer from the relatively low rates and more complicated backgrounds.

Neutrino decay modes: At the LHeC, there is similar sensitivity for LQ decay into both $e q$ and $\nu q$. At the LHC, in $p p$ collisions, LQ decay into neutrino-quark final states is plagued by huge QCD background. At the LHeC, production through eq fusion with subsequent $\nu q$ decay is thus very important if the complete pattern of LQ decay couplings is to be determined.

Coupling $\lambda$ : At the LHeC there is large sensitivity down to small values of the coupling $\lambda$. With less sensitivity, in $p p$ interactions at the LHC, information can be obtained from single LQ production and also from dilepton production via the $t$-channel LQ exchange. Since the single LQ production cross sections depend on both $\lambda$ and the flavour of the quark to which the LQ couples, determining $\lambda$ and this flavour requires $p p$ and $e p$ data.

\footnotetext{
${ }^{1}$ Single leptoquark production via photon splitting into an $e^{+} e^{-}$pair [16, which for masses of about $1 \mathrm{TeV}$ has a lower cross section than the quark-gluon induced processes, is not considered here.
} 

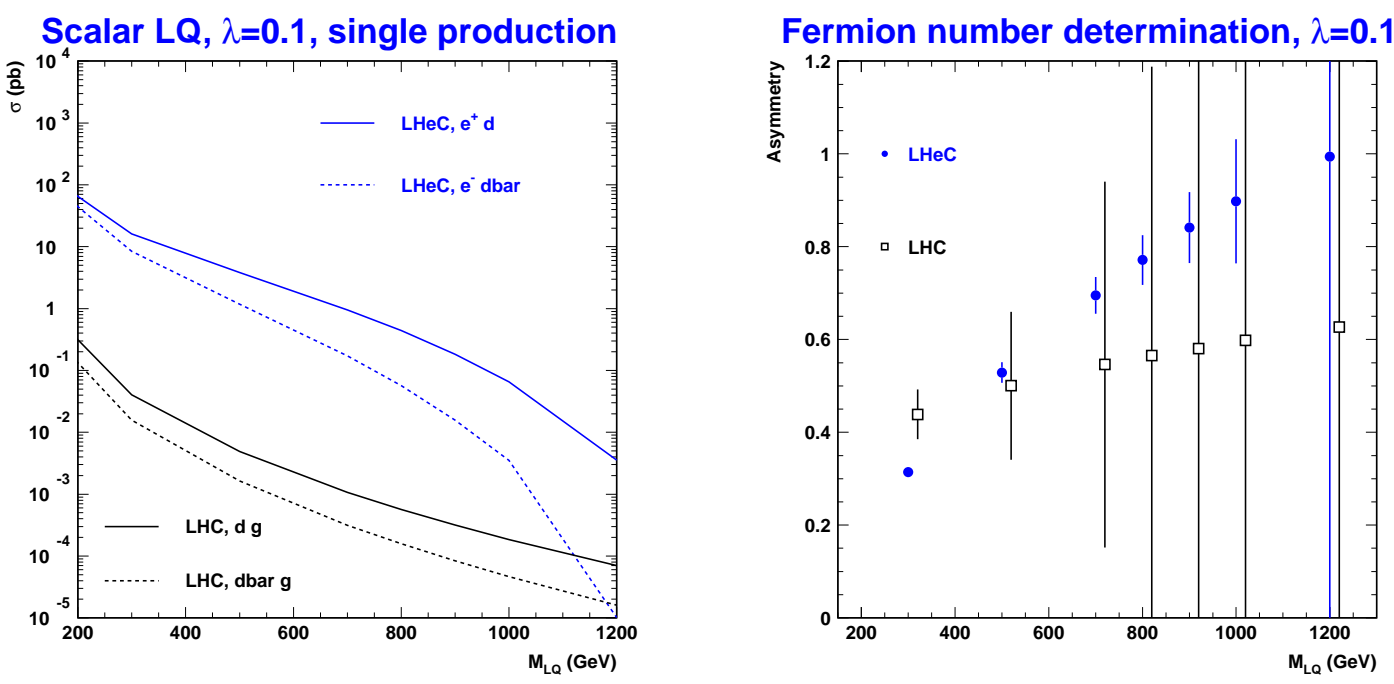

Figure 5: (Left) Single $L Q$ production cross section at the LHeC (top) and LHC (bottom), for a scalar $L Q$ coupling to $e^{+} d$ with a coupling $\lambda=0.1$; (Right) Asymmetries which would determine the fermion number of such a $L Q$, the sign of the asymmetry being the relevant quantity. An integrated luminosity of $100 \mathrm{fb}^{-1}\left(10 \mathrm{fb}^{-1}\right.$ per lepton charge) has been assumed for the $\mathrm{LHC}$ ( $\mathrm{LHeC}$ ).

Chiral structure of the LQ coupling: Chirality is central to the SM Lagrangian. Polarised electron and positron beams at the $\mathrm{LHeC}$ will shed light on the chiral structure of the LQ-e-q couplings. Measurements of a similar nature at LHC are impossible.

Table 1 summarises the observables [17] which could disentangle the various scalar LQ species at an ep machine. A similar discrimination power exists for vector LQs.

If Supersymmetry is manifest at $\mathrm{TeV}$ energy, in $e p$ interactions the associated production of squarks and sleptons ( $\tilde{e}$ and $\tilde{\nu_{e}}$ ) proceeds via the $t$-channel exchange of a neutralino or chargino. Fig. [6 shows that the rates can be sizeable at the LHeC when the sum of the squark and slepton masses is below $\sim 1 \mathrm{TeV}$. If squarks are relatively light, $\sim 500 \mathrm{GeV}$, selectron masses up to about $500 \mathrm{GeV}$ could be probed at the LHeC. This sensitivity may extend somewhat beyond the discovery reach for selectrons in $p p$ scattering at the LHC.

Charge asymmetries and, possibly, polarisation asymmetries could provide additional information on the couplings of the exchanged gauginos and on the mass difference between $\tilde{e}_{L}$ and $\tilde{e}_{R}$. Examples of such asymmetries are shown in Fig. 7, Light sfermions are assumed and the framework of the Minimal Supersymmetric Standard Model is used.

\footnotetext{
${ }^{2}$ Whether it is possible to achieve longitudinal polarisation in a $70 \mathrm{GeV} e^{ \pm}$beam in the LHC tunnel remains to be clarified.
} 


\begin{tabular}{|c|cccc|ccc|}
\hline & $S_{0, L}$ & $S_{1, L}$ & $\tilde{S}_{0, R}$ & $S_{0, R}$ & $S_{1 / 2, L}$ & $\tilde{S}_{1 / 2, L}$ & $S_{1 / 2, R}$ \\
\hline \hline$S_{0, L}$ & & $\beta_{\nu}$ & $P_{e}$ & $P_{e}$ & & & \\
$S_{1, L}$ & $\beta_{\nu}$ & & $P_{e}$ & $P_{e}$ & & & $e^{+} / e^{-}$ \\
$\tilde{S}_{0, R}$ & $P_{e}$ & $P_{e}$ & & - & & & \\
$S_{0, R}$ & $P_{e}$ & $P_{e}$ & - & & & & \\
\hline \hline$S_{1 / 2, L}$ & & & & & & - & $P_{e}$ \\
$\tilde{S}_{1 / 2, L}$ & & & $e^{+} / e^{-}$ & & - & & $P_{e}$ \\
$S_{1 / 2, R}$ & & & & & $P_{e}$ & $P_{e}$ & \\
\hline
\end{tabular}

Table 1: Discrimination between LQs with different quantum numbers in $e^{ \pm} p$ scattering with an electron beam polarisation $P_{e}$. The nomenclature of [18] has been used to label the different scalar $L Q$ species described by the model of Buchmüller, Rückl and Wyler [19], in which the branching ratio $\beta_{\nu}$ of the LQs to decay into $\nu+q$ is known.

\subsection{Physics of High Parton Densities (Low $x$ )}

The observations at HERA of the rise of the structure function $F_{2}\left(x, Q^{2}\right)$ and of its derivative $\partial F_{2} / \partial \ln Q^{2}$ as $x$ decreases [20] imply that at low $x$ the sea quark and the gluon distributions, respectively, of the proton increase dramatically. At low $x$ proton structure is thus driven by gluons and the formation of quark-antiquark pairs. While the charge of the proton is determined by its valence quarks, the kinetic and potential energy of gluons determines its mass. An understanding of quark-gluon dynamics is thus a key to the mass of the universe [21].

The sharp rise of the density of the gluons [22, 23] in the proton leads to the possibility of non-linear parton interaction effects [24]. A new dense state of parton matter is likely to exist, sometimes referred to as a Colour Glass Condensate [25], which is characterised by a high parton density and small coupling constant.

Much theoretical development in low $x$ physics is concerned with evolution equations which are the most appropriate approximation to a full solution to QCD (such as the BFKL [26], the CCFM [27], and the Balitzky Kovchegov equations [28]), and the incorporation of small $x$ resummation combining the classic DGLAP approach [29] with BFKL evolution [30]. DGLAP theory has been calculated to NNLO [31]. Physics at low $x$ has also been formulated using the colour dipole approach 32. However, there is still no universally accepted formulation of QCD at low $x$. This situation can be traced in large part to the fact that the kinematic reach of HERA is insufficient to establish unambiguously the existence of non-linear parton interaction effects and parton saturation phenomena [33, 34, 35].

Although the derivative $\partial F_{2} / \partial \ln x$ shows no evidence for a damping of the growth of the sea quark density with decreasing $x$, some effects observed at HERA in forward jet production [36] and in azimuthal (de)correlations [37] seem to indicate departures from the conventional radiation pattern in QCD. Future progress requires the substantial increase of the kinematic reach to low $x$ (Fig.8) which is made possible by the LHeC. 

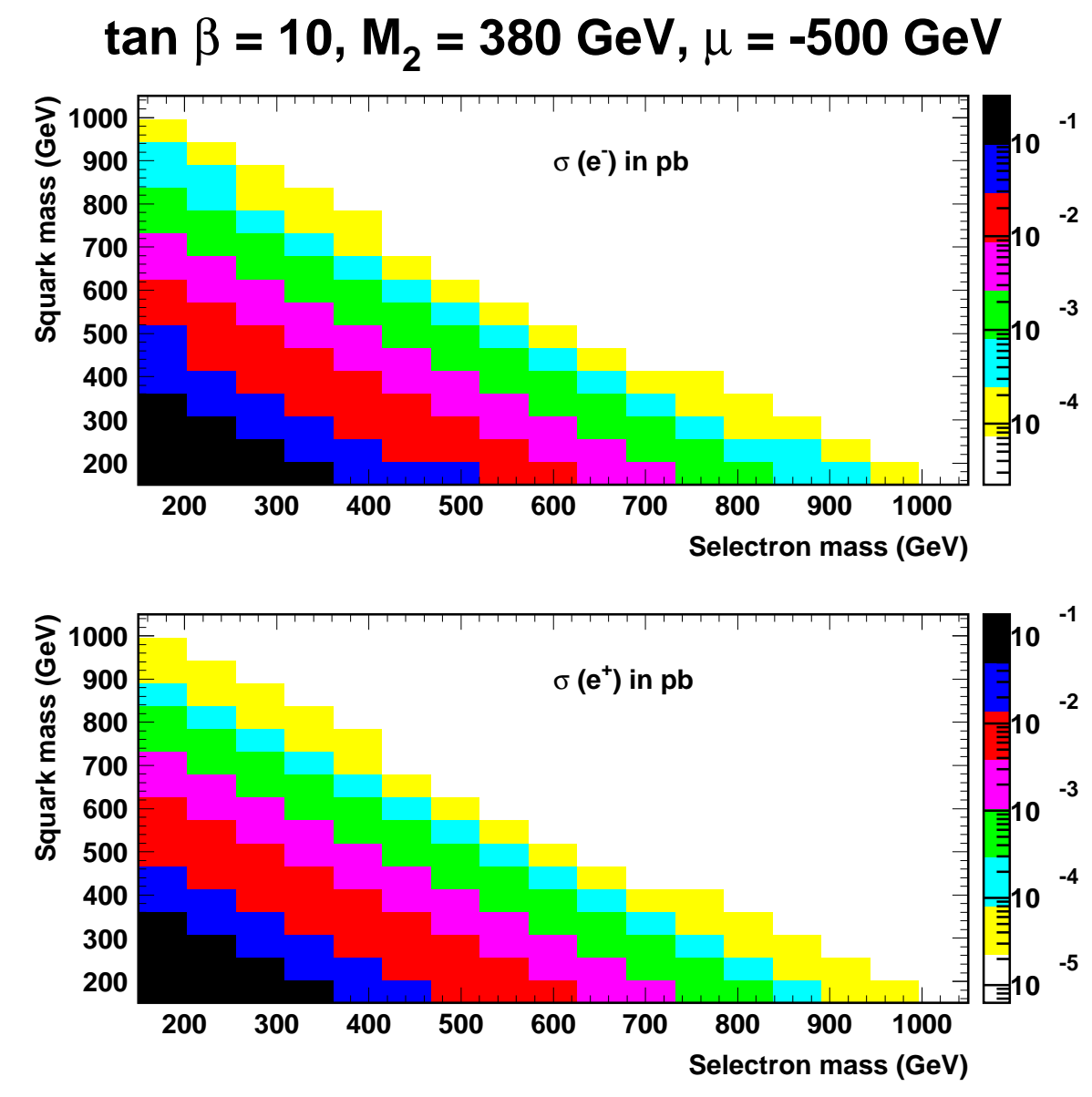

Figure 6: Production cross section (right scale, in pb) of a selectron-squark pair at the $L H e C$, for example values of MSSM parameters. It is assumed that $\tilde{u}_{L}, \tilde{u}_{R}, \tilde{d}_{L}$ and $\tilde{d}_{R}$ are degenerate, as are $\tilde{e}_{R}$ and $\tilde{e}_{L}$.

At the LHC measurements will be performed of the interaction of nuclei, in for example $\mathrm{Pb}-\mathrm{Pb}$ collisions, with the aim of investigating a high density parton phase (Quark Gluon Plasma). An unambiguous determination of the nuclear parton distributions in the domain accessed with these measurements, which is possible in $e A$ scattering at the LHeC, will be important if the equilibrium of the nucleon (colour singlet) and parton (colour non-singlet, QGP) phases are to be understood. A complete unknown is the gluon distribution in nuclei at low $x$ (Fig.99) 38].

If, as one expects, the evolution at low $x$ proceeds differently from DGLAP based predictions, there are important consequences for high energy physics, which reach beyond the intrinsic questions of low $x$ theory. These may include

- the observation of the black disc limit of high energy scattering [39], i.e. of the unitarity limit, which causes cross sections at high energy to saturate resulting in 


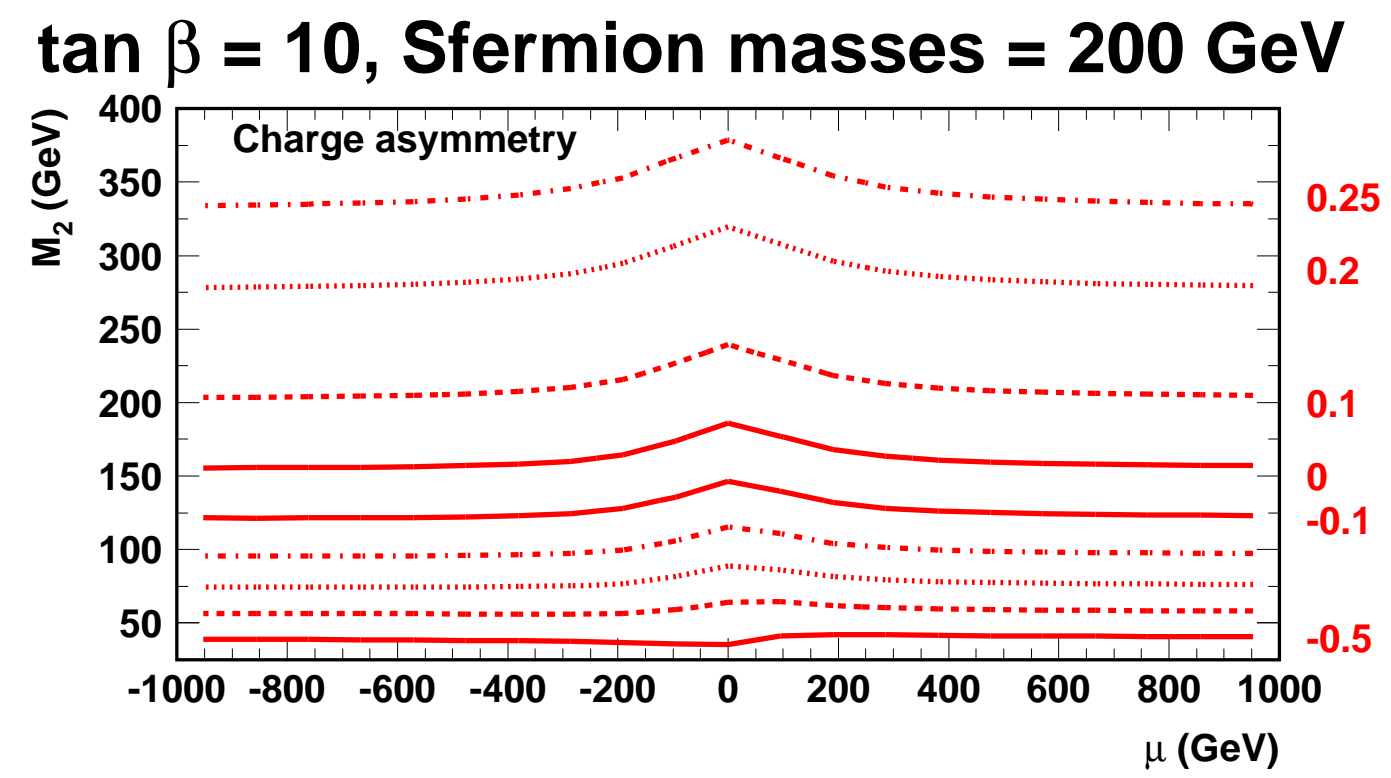

Figure 7: Charge asymmetry of the ẽ $\tilde{q}$ production cross section at LHeC in the $M_{2}-\mu$ plane in the MSSM. Light sfermion masses are assumed. The numerical values on the right of the plot correspond to the asymmetry values of the contours.

- new, possibly radically different, predictions for high energy cosmic ray and neutrino physics, which currently are based on large extrapolations [40], and also

- a different perspective on physics in the forward region of $p p, p A$ and $A A$ interactions at the LHC.

\section{Precision Quantum Chromodynamics}

QCD is one of the cornerstones of our understanding of the physics of the universe, which is encapsulated in the Standard Model (SM). Like the electroweak sector of the SM, its predictive power depends on the accuracy with which its gauge coupling is known and on our ability to make predictions and compare them with experiment for a variety of hadronic phenomena. Lepton-hadron interactions, in which hadronic matter is probed deeply in a well understood and point-like manner, is an extremely powerful means of achieving these aims.

There follow a few examples, in which at the $\mathrm{LHeC}$, because of the precision of an $e p$ experiment and because of kinematic reach, one can foresee a major improvement in the accuracy, with which QCD can be tested, quantified and developed further. 


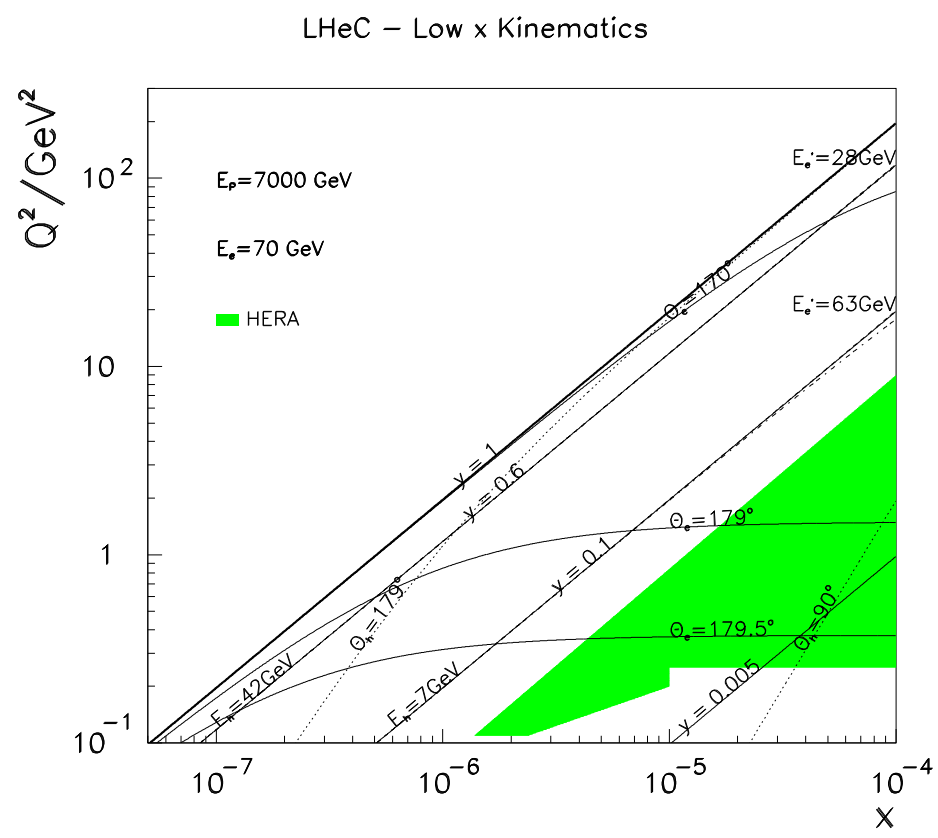

Figure 8: Kinematics of ep scattering at the LHeC at low $x$. Access to the $Q^{2}$ region down to $1 \mathrm{GeV}^{2}$ requires a dedicated interaction region with acceptances extended to about $179^{\circ}$, with respect to the proton beam direction, i.e. an extended "backward detector". The shaded area illustrates the region of kinematic coverage in NC scattering at HERA, which has been extended below $x \sim 10^{-4}$ with special techniques, namely a detector attached to the beam pipe backward of the main apparatus and also by shifting the interaction vertex into the proton beam direction.

\subsection{Structure Functions and Partons for the LHC}

Precision measurements at the LHeC of the neutral current (NC) and charged current (CC) deep-inelastic scattering cross sections (Fig.10) are pivotal in building a successful physics programme at the LHC. Large electroweak effects, which add quark flavour and matter-antimatter sensitivity, are present (Fig.11). The primary purpose of these measurements is to extract a number of important structure functions of the proton, and hence to determine a comprehensive set of parton density functions (pdf) for the nucleon at the LHC energy scale, thereby avoiding the uncertainties introduced in the evolution of measurements from the HERA energy scale. Important examples where new constraints are needed are the behaviour of the sea and valence quarks at low $x$, which can be obtained from a measurement of the $\gamma Z$ interference structure function $x G_{3}$ (Fig.12), and the limit of the $u / d$ ratio at large $x$, obtainable from a measurement of charged current $e^{ \pm} p$ scattering (Fig.13).

The accuracy with which the parton distributions can be determined contributes directly to the sensitivity to new physics at both the LHC and the LHeC. An example is the envisaged measurement to $1 \%$ of the luminosity in $p p$ interactions at the LHC using $W$ and $Z$ production [44]. So as to cover the rapidity plateau region at the LHC, it is necessary to know the pdfs of quarks and gluons [45] in the Bjorken- $x$ range $10^{-4}$ 


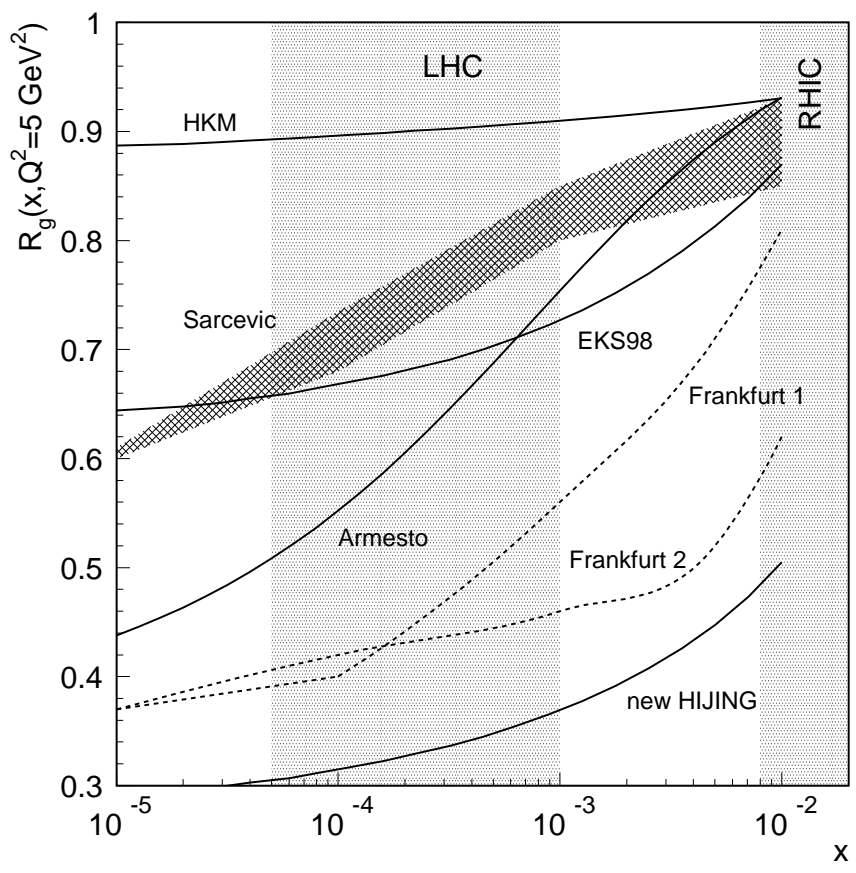

Figure 9: Ratios of gluon distribution functions in lead $(\mathrm{Pb})$ relative to the gluon distribution in the proton using different models, at $Q^{2}=5 \mathrm{GeV}^{2}$.
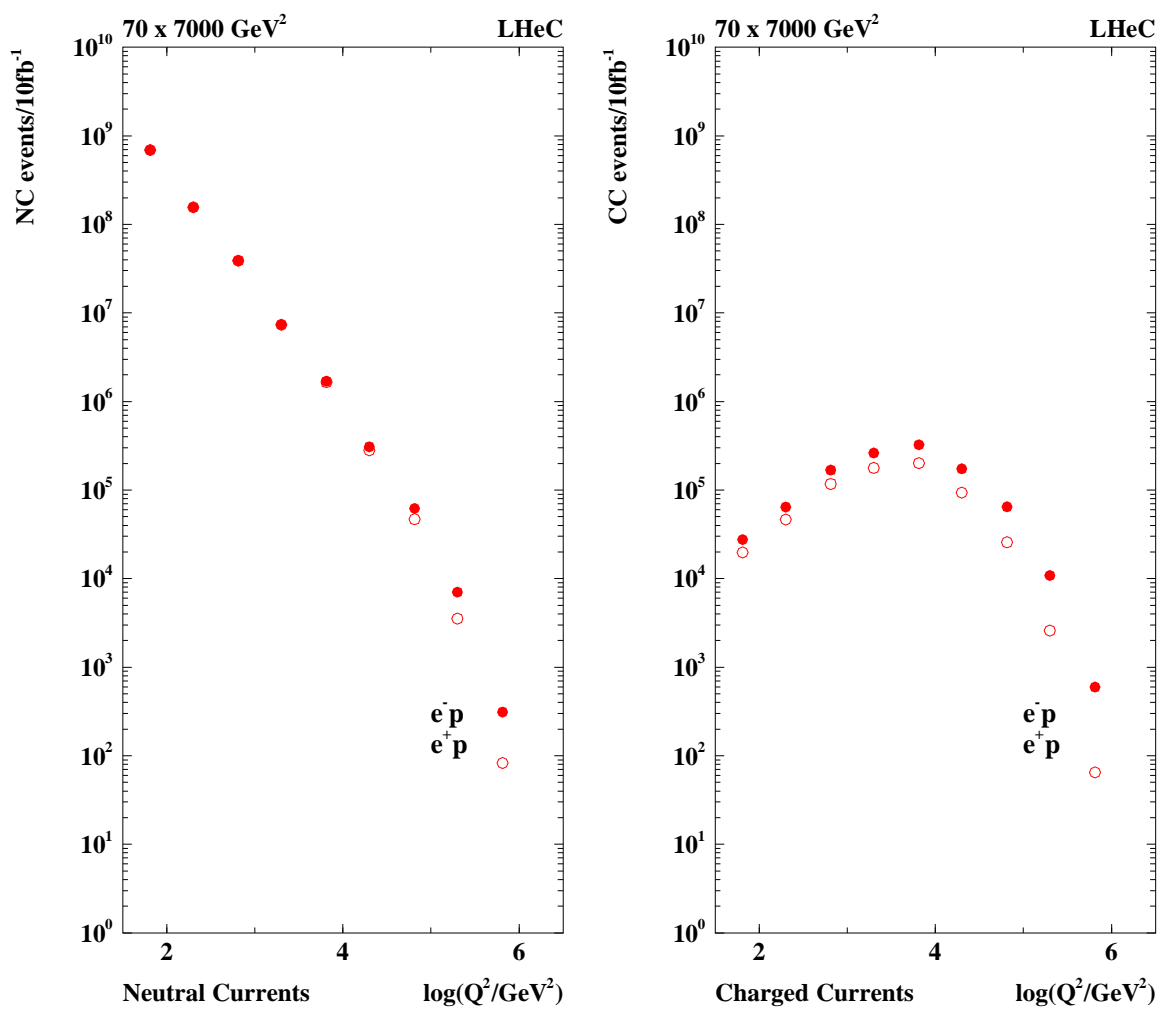

Figure 10: Event rates per $10 \mathrm{fb}^{-1}$ in neutral (left) and charged (right) current $e^{ \pm} p$ scattering at the LHeC for the high lumnosity acceptance, Section 5. At design luminosity such statistical sensitivity may be accumulated within one year of operating the LHeC. 

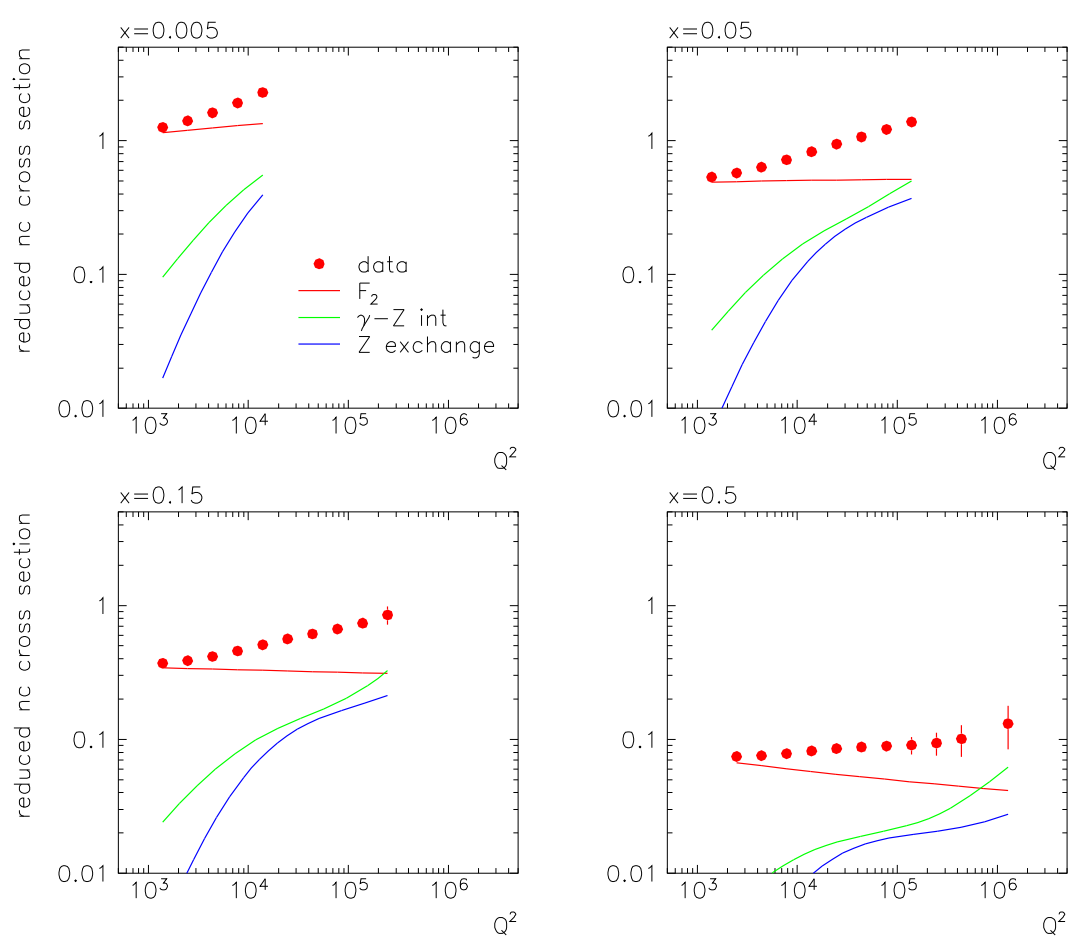

Figure 11: Simulation of a measurement in unpolarised $e^{-} p$ scattering of the reduced $N C$ cross section, $\sigma_{r}=d \sigma / d x d Q^{2} \cdot Q^{4} x / 2 \pi \alpha^{2}\left(1+(1-y)^{2}\right)$. In the one-photon exchange approximation $\sigma_{r}=F_{2}$ for $F_{L}=0$. With rising $Q^{2}$ the effects due to the exchange of weak bosons become sizeable such that, for example, the reduced cross section does not decrease at large $x$ any more, as would be the case for pure photon exchange, solid (red) line. The luminosity used here is only $200 \mathrm{pb}^{-1}$. Accurate measurements at the $\mathrm{LHeC}$ in the rapidity plateau region of the $\mathrm{LHC}$ can thus be made using data from a very short running period, namely less than two weeks at design luminosity.

to 0.1 at $Q^{2}=M_{Z, W}^{2} \simeq 10^{4} \mathrm{GeV}^{2}$ with adequate precision. This range is covered directly by the LHeC. If HERA data alone are to be used, a large extrapolation in $Q^{2}$ is necessary, which requires an accurate understanding of perturbative QCD. The theory underpinning the extrapolation is subtle and far from unambiguous [46]. At large $x$, resummation effects are important. At low $x$ the usual assumption of DGLAP evolution must ultimately break down. Furthermore, the parton dynamics may be nonlinear (see Section 2.2), and multiple interactions may have to be taken into account when extrapolating to high energy [47].

At LHC, heavy quarks play an essential role in QCD dynamics, and their contributions to proton structure must therefore be understood well. In particular the bottom quark distribution needs to be known rather accurately because $b$ quarks contribute substantially to the production mechanisms for new physics. With rising $Q^{2}$ the fraction of the heavy quark contributions increases (Fig.14), for $b$ quarks from a few per mil near threshold at HERA [49] to about $5 \%$ at the LHeC. Thus with silicon vertex detectors, and taking advantage of the small beam spot size $\left(15 \cdot 35 \mu \mathrm{m}^{2}\right)$, very accurate measurements of the beauty and the charm quark densities are possible in a very wide 


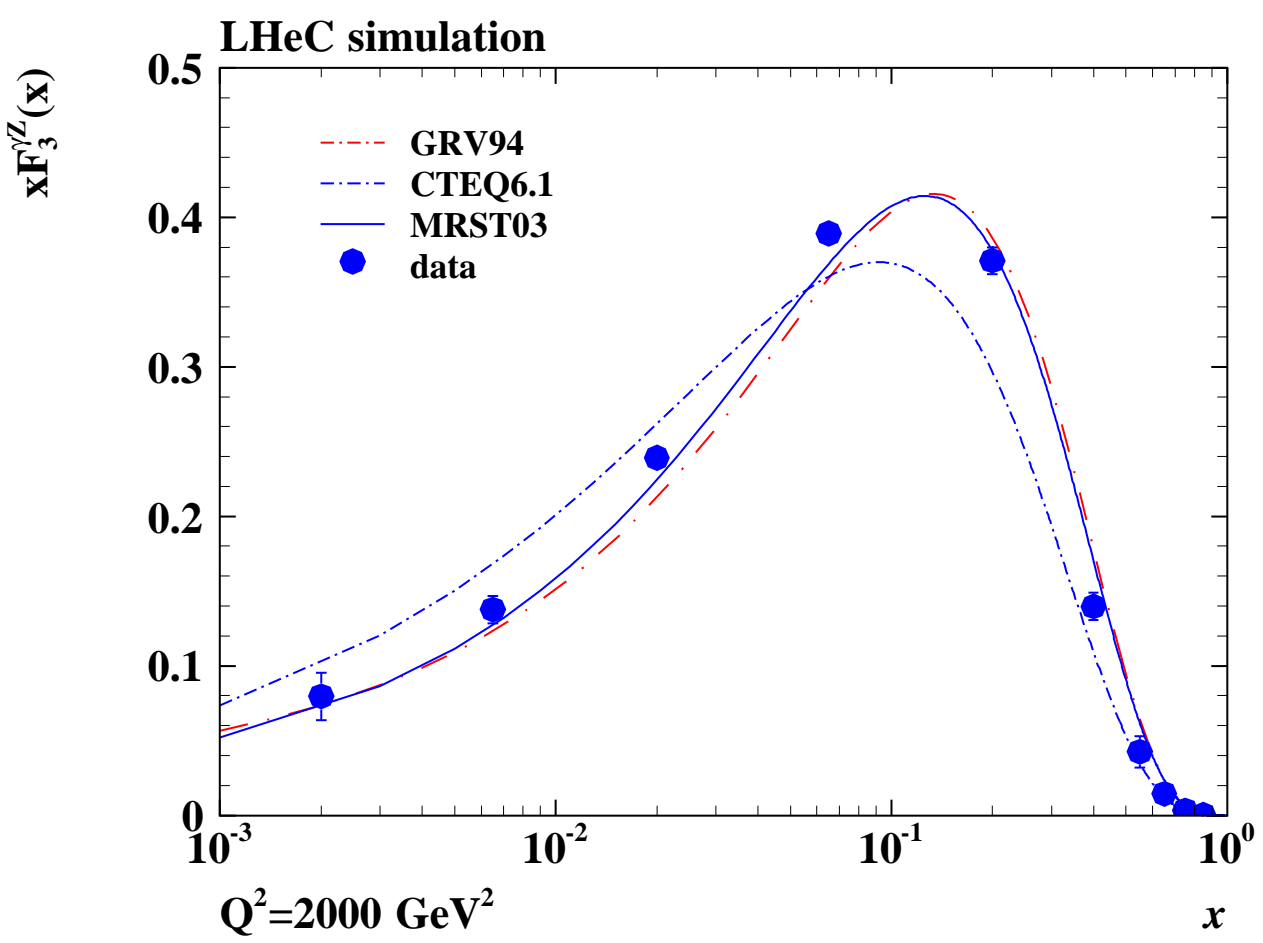

Figure 12: Simulation of a measurement of the neutral current interference structure function $x G_{3}=x F_{3}^{\gamma Z}$ from an $e^{ \pm} p N C$ charge asymmetry measurement assuming a luminosity of $10 \mathrm{fb}^{-1}$ per beam setting. This function is defined as [42] $x G_{3}=2 x\left[a_{u} e_{u}(U-\bar{U})+a_{d} e_{d}(D-\bar{D})\right]$, where $U(D)$ denotes the sum of the up (down) quark distributions in the proton. For the first time one thus has direct access to the antiquark-sea (a?)symmetry at low $x$ in the deep inelastic region. It is usually assumed that antiquarks and sea quarks are equal to each other, e.g. $\bar{u}=u_{s}$ and $s=\bar{s}$. This assumption is common to all pdf parameterisations used here, so that $x G_{3}$ tends to zero at low $x$. Recent analyses of the NuTeV anomaly, however, suggest a possible difference between strange and anti-strange quark distributions [43]. Symmetry implies that $x G_{3}=\left(2 u_{v}+d_{v}\right) / 3$, which is expected to drop to zero at low $x$ and to be roughly independent of $Q^{2}$. The data points are thus projected to an average $Q^{2}$ to display the accuracy of the measurement. From the very high $Q^{2}$ region one will be able to derive a measurement over 3 orders of magnitude in $x$. Note that at HERA this function can only be measured down to $x \simeq 0.02$ and with much less accuracy since the $Q^{2}$ values involved are smaller, i.e. electroweak effects weaker, and the luminosity is inferior to what is projected for the $\mathrm{LHeC}$. 

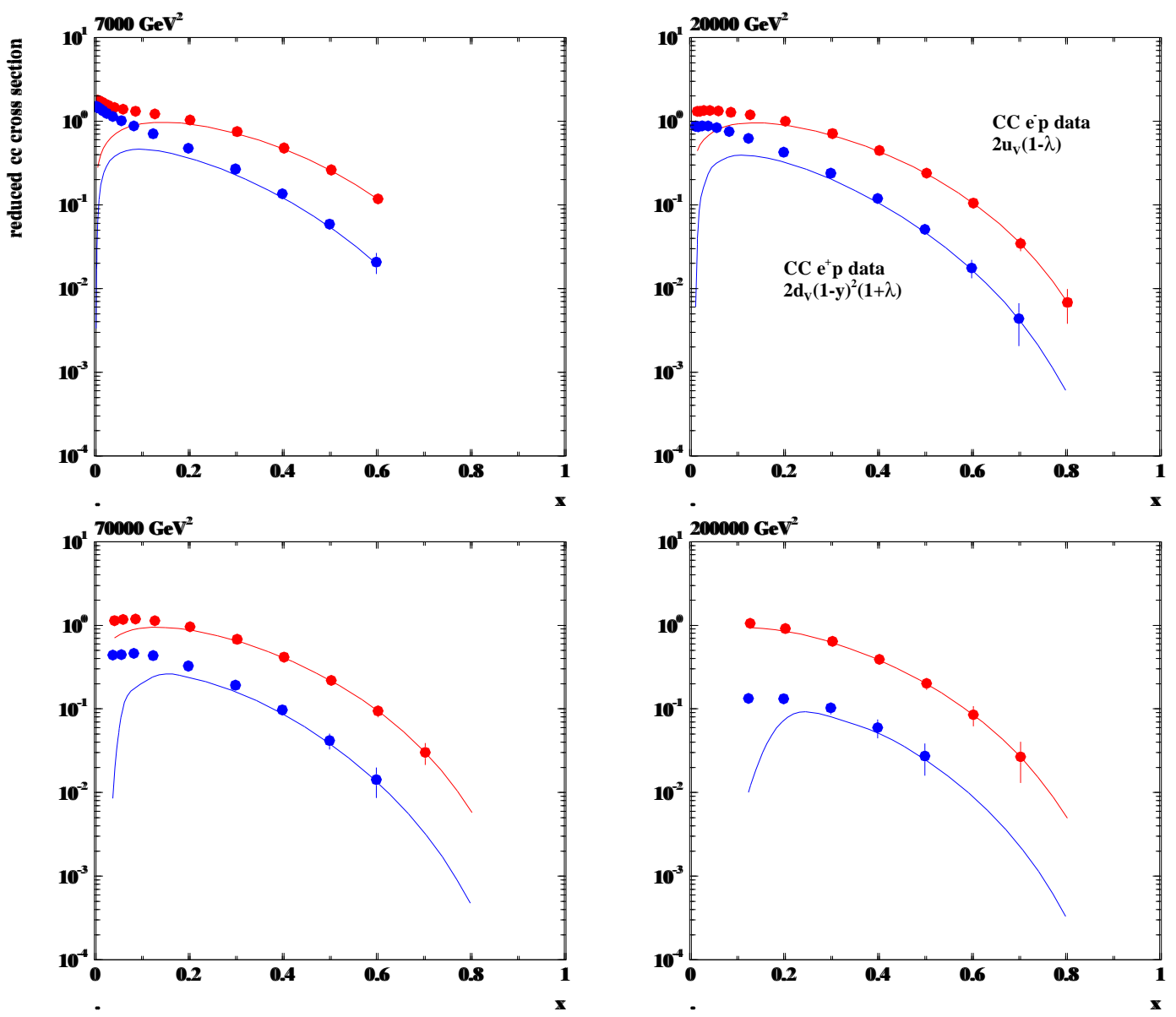

Figure 13: Reduced charged current cross sections with statistical uncertainties corresponding to $1 \mathrm{fb}^{-1}$ in electron (top data points, red) and positron (lower data points, blue) proton scattering at the $\mathrm{LHeC}$, simulated for the low $x$ detector configuration, see Section 5. The curves are determined by the dominant valence quark distributions, $u_{v}$ for $e^{-} p$ and $d_{v}$ for $e^{+} p$. In the simulation the lepton polarisation $\lambda$ is taken to be zero. The valence quark approximation of the reduced cross section is seen to hold already at $x \simeq 0.2$ and a rather accurate determination of the $u / d$ ratio up to large $x$ appears to be feasible at very high $Q^{2}$. 


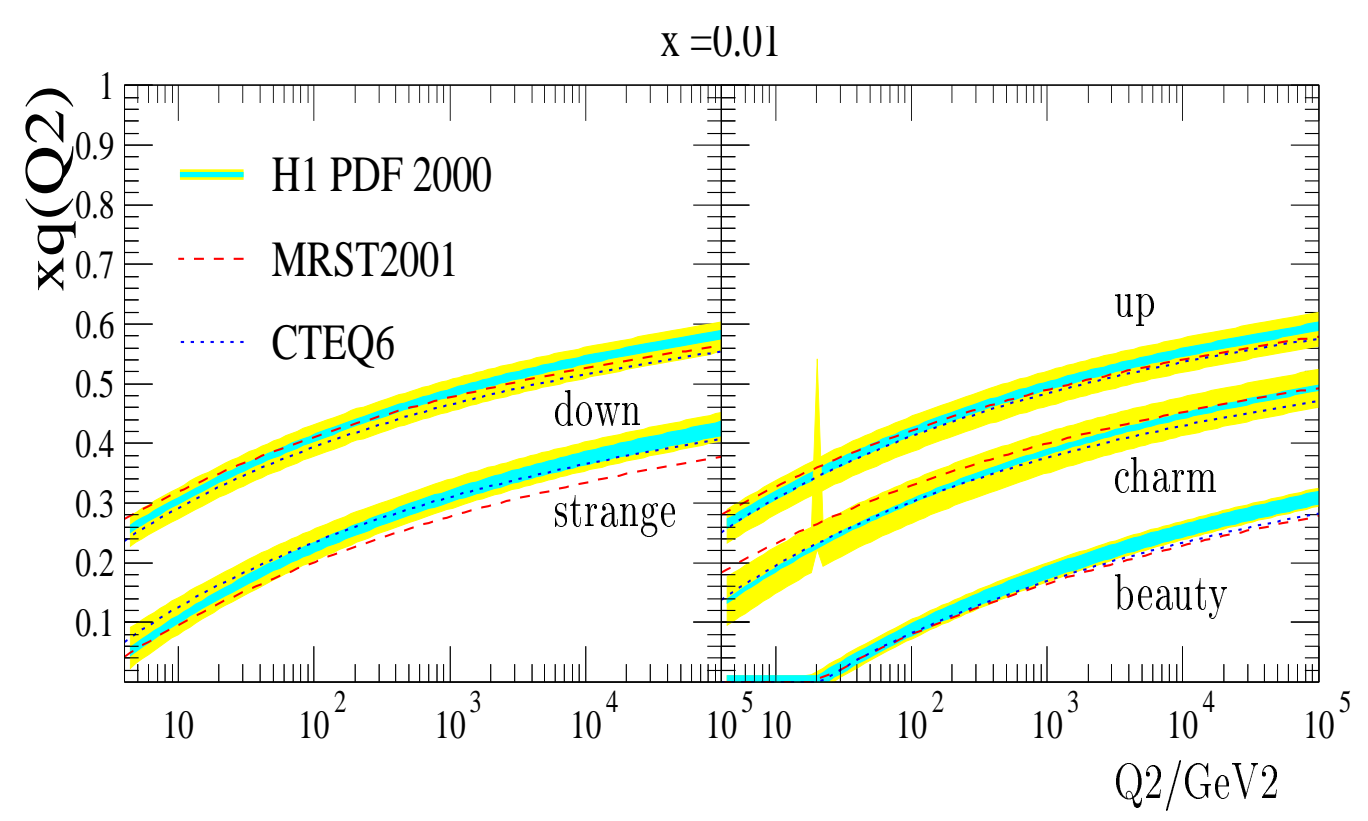

Figure 14: The $Q^{2}$ evolution of the sea quark distributions, for $x$ within the rapidity plateau at the LHC, as predicted using the NLO DGLAP pdf analyses of H1, MRST and CTEQ. At $x=0.01$, the $\mathrm{LHeC}$ will cover a $Q^{2}$ range up to $10^{4} \mathrm{GeV}^{2}$, by which point the heavy quark distributions become similar in size to the light quarks. The complete determination to high accuracy of the parton distributions is essential for searches for new physics at the $\mathrm{LHC}$ and also for measuring its "partonic" luminosity from the $W, Z$ boson production rates.

range of $Q^{2}$ and $x$. The greatly enhanced kinematic reach at the LHeC, together with vertex flavour tagging, will make possible measurements of high $x$ strange and beauty quark densities in the proton using the couplings $s W \rightarrow c$ and $b W \rightarrow t$ respectively.

Thus, at the LHeC determinations at a new level of precision and to previously unexplored $x$ values of all quark distributions in the proton can be anticipated, and, with the combination of the LHeC and HERA structure function measurements, a determination of the gluon distribution in the proton with unprecedented accuracy over an extended range of $x$ and of momentum transfer will also result.

\subsection{Strong Coupling Constant}

The strong coupling constant $\alpha_{s}$ is currently known to $1-2 \%$ experimental error. This is many orders of magnitude worse than the determination of the fine structure constant and the Fermi constant, which are known at the level of $10^{-9}$ and $10^{-5}$, respectively. The gravitational constant is known to $0.1 \%$. In unified theories the electromagnetic, weak and strong couplings are expected to approach a common limit at some large unification scale. Presently, the accuracy of such extrapolations is limited by the uncertainty with which $\alpha_{s}$ is known (Fig. 15 [50]). Precision tests of QCD and comparisons 
with lattice QCD calculations [51] require a significant improvement in the knowledge of $\alpha_{s}$.

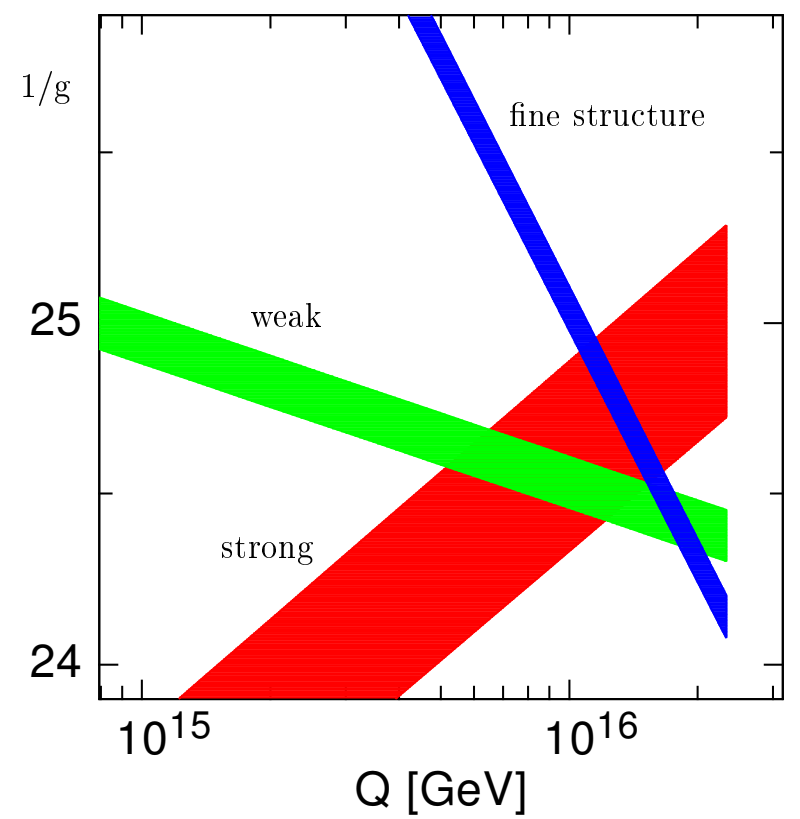

Figure 15: Two-loop extrapolation of the inverse coupling constants of $U(1), S U(2)$ and $S U(3)$ to the unification point, defined as $1 / g_{1}=1 / g_{2}$, in the MSSM model using the $\overline{D R}$ scheme, where $g_{1}\left(g_{2}\right)$ denotes the electromagnetic (weak) coupling constant. The uncertainty is dominated by the rather moderate knowledge of the strong coupling constant. Improvements on the measurement of $\alpha_{s}$ are expected also from the Giga $Z$ mode at the ILC [50].

Deep-inelastic scattering is a well defined process theoretically [1, 52, and has recently been calculated to NNLO [31]. The determination of the strong coupling constant in DIS requires the simultaneous determination of the gluon distribution, $x g$, and the quark distributions. At HERA $\alpha_{s}$ is determined to within an experimental accuracy of about $1 \%$ [53]. With the inclusion of the LHeC data, the experimental accuracy is expected to reach a few per mil.

At such a high level of experimental accuracy, it will be necessary to reassess many theoretical and phenomenological problems of crucial importance to our understanding of QCD and how to use it, for example, the treatment of the renormalisation scale $\left(\mu_{r}\right)$ uncertainty. By convention 3 , one still varies $\mu_{r}^{2}$ between $Q^{2} / 4$ and $4 Q^{2}$, which at NLO introduces an uncertainty on $\alpha_{s}$ of about $5 \%$ and at NNLO is estimated to be about $1 \%$ [31]. Further examples of theoretical issues which appear at the new level of accuracy with LHeC data are the treatment of heavy flavours in QCD evolution [54] and the limits to the validity of the DGLAP approximation in deep-inelastic scattering [55].

\footnotetext{
${ }^{3}$ Such a large variation of the renormalisation scale is already not supported in NLO QCD analyses of the HERA structure function data of H1 22] and ZEUS 23] and the prescription for estimating the resulting theoretical uncertainty of $\alpha_{s}$ needs to be reconsidered.
} 


\subsection{Hard Diffraction}

As discussed in the previous sections, a detailed understanding of physics in the LHC energy range will require substantial developments in the knowledge of Quantum Chromodynamics in the high density, low $x$, environment. Low $x$ studies at HERA and the Tevatron have shown clearly that diffraction has to be an integral component of any successful low $x$ theory. Furthermore, the contrast between non-diffractive DIS, where

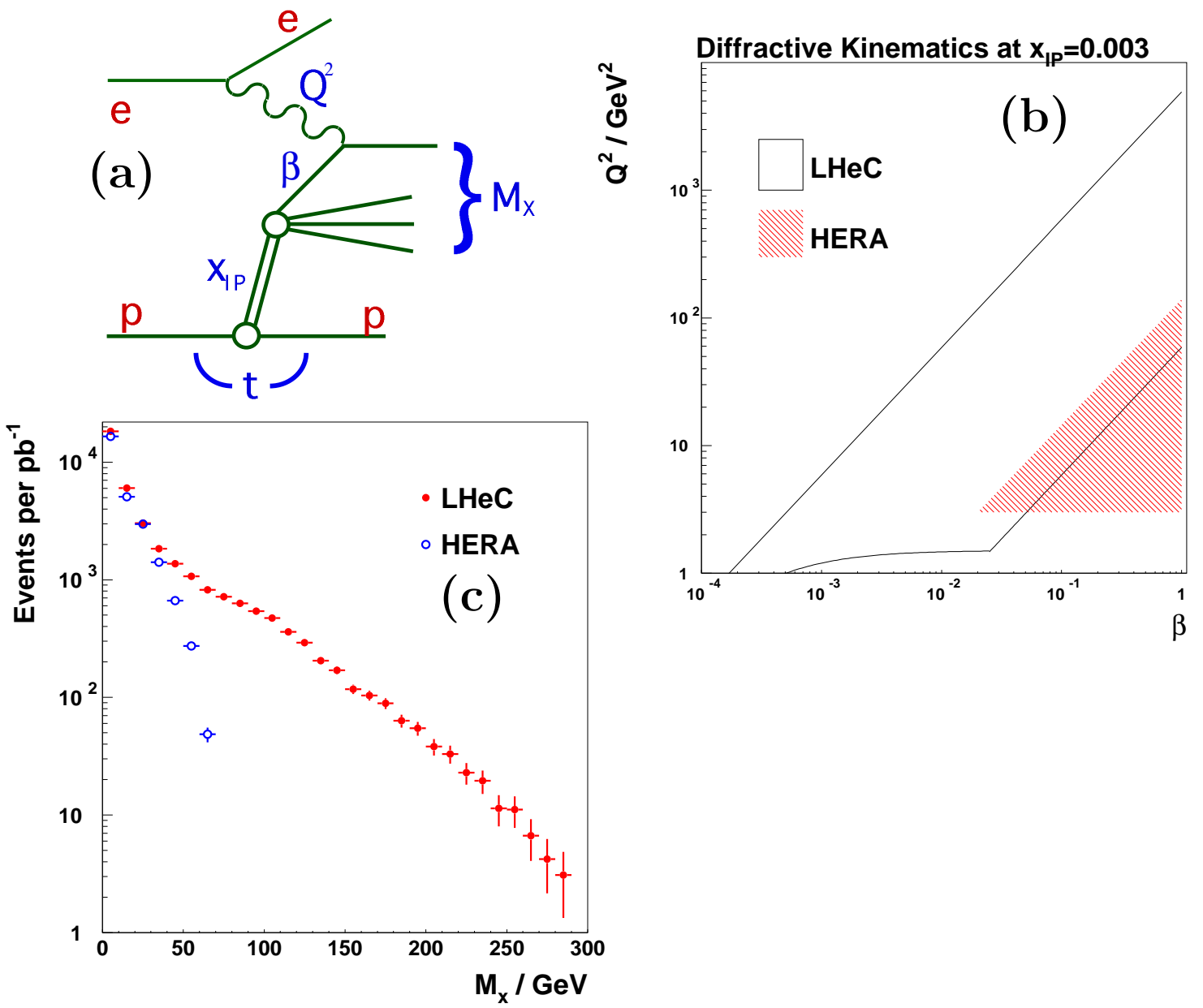

Figure 16: (a) Feynman diagram for diffractive DIS, indicating the kinematic variables used in the text. (b) Comparison of the kinematic region in $\beta$ and $Q^{2}$ accessible at $H E R A$ and $L H e C$ for an example $x_{\mathbb{P}}=0.003$. The LHeC region corresponds to $0.01<$ $y<1$ and $\theta_{e}^{\prime}<179^{\circ}$. Similar extensions are available over the full range of $x_{\mathbb{P}}<0.05$ which is relevant to diffraction. (c) Monte Carlo simulation, comparing the diffractive mass distributions at HERA and the $\mathrm{LHeC}$ available for studies of the hadronic final state with the selection $Q^{2} \geq 3 G e V^{2}, 0.1<y<0.9$ and $x_{\mathbb{P}}<0.05$.

the proton is rather violently broken up, and diffractive DIS where the proton remains intact, offers a rare experimental window on the mechanism which confines quarks within hadrons.

One of the biggest successes of HERA has been the development of the understanding of diffraction in QCD through the study of the diffractive DIS process (Fig. 16a) 
$e p \rightarrow e X p$ [57]. In this process, the proton remains intact, but loses a fraction $x_{\mathbb{P}}$ of its longitudinal momentum in the form of some net colourless partonic system. The virtual photon probes this colourless system, coupling to a quark with a fractional momentum $\beta$, in much the same way as the whole proton is probed in inclusive DIS, and producing a diffractive system of mass $M_{\mathrm{X}}$. The new kinematic regions and very large luminosities possible at the LHeC make it ideal for further precision study of hard diffraction. Figures $16 \mathrm{~b}$ and $16 \mathrm{c}$ illustrate the dramatic improvements in diffractive kinematic coverage at the LHeC compared with HERA.

A general framework to describe diffractive DIS is provided by a QCD factorisation theorem [58], which allows "diffractive parton distributions" (dpdfs) to be defined. Such dpdfs have been extracted from the diffractive structure function $F_{2}^{D}$ and its scaling violations at HERA [59]. However, unlike the case of inclusive scattering, the dpdfs are only applicable to DIS processes. Large and very poorly constrained "survival" factors are needed before dpdf's can be used to predict $p p$ scattering processes. Testing the factorisation properties and dpdfs extracted at HERA has therefore only been possible thus far by predicting final state DIS observables such as jet or charm cross sections. This approach is heavily limited by the relatively low accessible $M_{\mathrm{X}}$ values at HERA (Fig. 16r), which imply that jets can be studied only at uncomfortably low $p_{t}$ values $\left(\leq M_{\mathrm{X}} / 2\right)$ and that charm cross sections are frustratingly small. The much larger invariant masses accessible at the LHeC would circumvent these problems and would open up new and complementary channels to study, such as diffractive beauty production and diffractive electroweak gauge boson production.

The only way of making significant further constraints and extensions to the dpdfs and studying their QCD evolution is at a higher energy facility such as the LHeC. With the high available luminosities, it will be possible to make measurements in the HERA $\beta$ range, but at larger $Q^{2}$ (Fig. 16 $\mathrm{b}$ ), which would provide a unique opportunity to test the applicability of DGLAP evolution to diffraction.

Fig. 16b also shows a substantial extension towards lower $\beta$ at the LHeC, similar to that available to lower $x$ in the inclusive case. As discussed above, it is almost certain that new QCD physics associated with the taming of the growth of the gluon density will be observed in this region. The diffractive exchange is often modelled as being derived from a pair of gluons, in which case any novel effects observed in the gluon density should be amplified in the diffraction case. Such measurements of the QCD structure of diffraction are fundamental, amounting to direct experimental measurements of the partonic structure of the vacuum and its quantification in terms of distribution functions.

\subsection{Final State Physics}

The hadronic final state in deep-inelastic interactions probes the QCD cascade, and therefore is a unique and important probe of the chromodynamics of confinement. While predictions based on the DGLAP evolution equations provide a good description 
of the current inclusive DIS data, there are indications that this approach might not be sufficient. For example, HERA data on forward jet production at low $x$ show that non $k_{t}$-ordered contributions play an important role. At the LHeC the kinematic domain is extended to much lower $x$ values, providing much greater sensitivity to the topological features of the cascade. With LHeC data various approaches for modeling QCD cascades could thus be distinguished for the first time (Fig.17) and constraints on the unintegrated parton densities [60] could be obtained. In addition, the increase of the centre of mass energy with respect to HERA will lead to a much larger cross section for heavy flavor production. This would allow the $b$ and $c$ photoproduction cross sections to be measured up to large transverse momentum, providing a much larger level-arm when comparing the measurements to the QCD predictions of the collinear or $k_{t}$-factorisation approach [9].

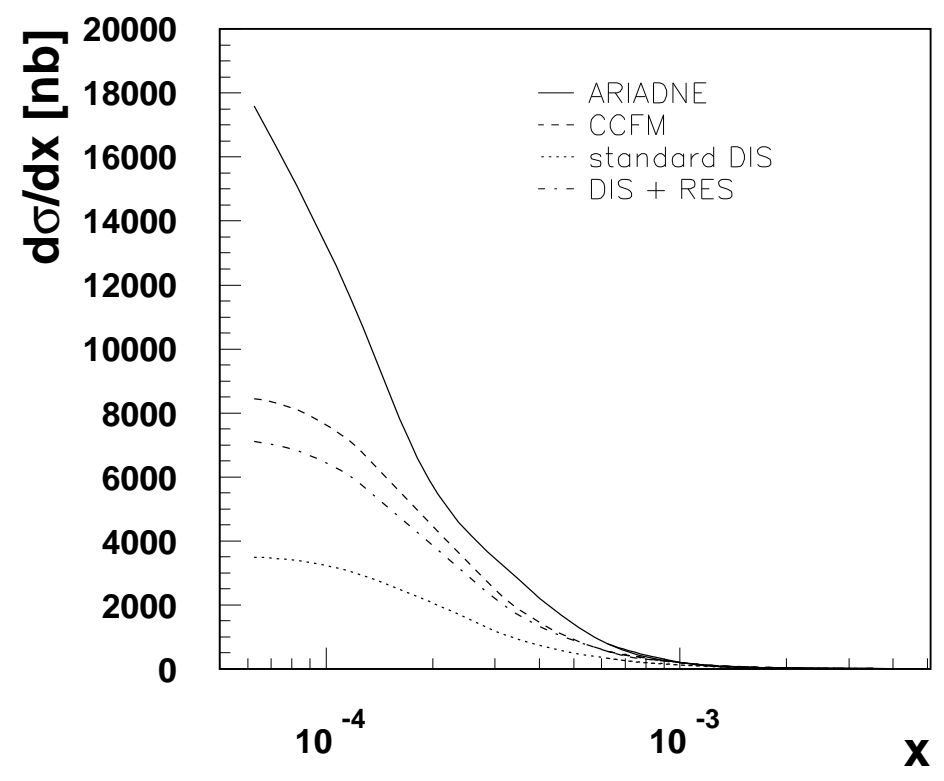

Figure 17: Forward jet cross section as a function of $x$ predicted by different models for the pattern of parton radiation, for $0.5<p_{T}^{2} / Q^{2}<2$ and minimum polar jet angle of $1^{\circ}$ as is envisaged for the low $x$ interaction region at the $\mathrm{LHeC}$. The measurements at HERA are limited to $x \geq 2 \cdot 10^{-3}$, a range in which the resolved photon effects may still mimic non- $k_{t}$ ordered parton emission. The predictions of ARIADNE and the CCFM equation become largely different only at lower $x$ values, below the kinematic range accessed by HERA, see [9].

The hadronic structure of the photon and its interaction with hadronic matter have long been something of a conundrum. The advent of both $e^{+} e^{-}$and ep colliders in the last two decades, and the huge increases in kinematic reach which have resulted, have meant that it is now possible to see how QCD contributes to the interplay of photon structure and photon interaction dynamics. As a result there is now a phenomenology of hadronic photoproduction $(e p)$ and two-photon production $\left(e^{+} e^{-}\right)$. A distinction between "resolved" and "direct" interactions can be made by means of a Bjorken- $x$ like variable $x_{\gamma}$, which specifies the fraction of the photon momentum participating in the interaction. Resolved photon interactions $\left(x_{\gamma}<1\right)$ depend on the 
structure of the photon and direct photon interactions $\left(x_{\gamma}=1\right)$ correspond to the direct coupling of the photon in a hadronic interaction.

Photoproduction measurements at HERA of hard QCD phenomena (final state transverse momentum squared $p_{T}^{2}>Q^{2}$ ) are used to extract structure functions of the photon over a substantial range of Bjorken- $x$ and of photon dimension $(\propto 1 / Q)$. Features of photon structure, which derive from its underlying $\gamma \rightarrow q \bar{q}$ splitting, are now established, and the subtle interplay between the hard scales $\left(Q^{2}, p_{T}^{2}\right.$, and when involved a heavy quark mass) are emerging as the photon virtuality, that is its size, changes.

As a result, there now exists a rather comprehensive picture of photon-hadron coupling in terms of QCD for values of $x_{\gamma}$ down to about 0.1. Furthermore, the ability to "tune" the nature of the photon interaction between direct and resolved (hadronlike) now begins to throw light on issues in hard hadronic interactions such as multiple parton interactions which would break QCD factorisation. Measurements at LHeC will extend hadronic photoproduction measurements to $x_{\gamma} \simeq 0.01$ at large momentum transfer, and thus will begin to reveal the properties of the gluon-dominated region of photon structure [9], that is of a flavour singlet object of variable dimension $\left(Q^{2}\right)$.

Prompt photon production, ep $\rightarrow \gamma X$, the deep inelastic Compton scattering process, is also sensitive to QCD and photon structure. In the foward region, $\eta_{\gamma}>0$, this process is dominated by the reaction $g q \rightarrow \gamma q$ with a cross section which is an oder of magnitude higher at LHeC than at HERA and which extends to larger $p_{t}$ of the photon. Prompt photon production at the LHeC will thus explore the gluon content of the photon [61].

A new way of probing hadronic matter involves the physics of deeply virtual Compton scattering (DVCS), ep $\rightarrow e p \gamma[62$. Here a parton in the proton absorbs the virtual photon, emits a real photon and the proton ground state is restored. In this process, two gluons at low $x$ (at collider experiments) or two quarks at larger $x$ (as in fixed target experiments), carry different fractions of the initial proton momentum. The DVCS process thus measures generalised parton distributions (GPDs) [63] which depend on two momentum fractions $x$ and $\xi$, as well as on $Q^{2}$ and the squared fourmomentum transfer $t$ at the proton vertex. The DVCS process interferes with BetheHeitler scattering, which allows parton scattering amplitudes to be measured, rather than just cross sections in the form of standard pdfs.

The huge increases in kinematic reach and luminosity with the LHeC will make it possible to build an understanding of proton structure in terms of both transverse (impact parameter or $p_{T}$ ) and longitudinal (rapidity or Bjorken- $x$ ) dimensions. Such proton "tomography" [64] will thereby provide a deeper understanding of proton structure. The possibility that DVCS at the LHeC may also be measured in charged current interactions would, as in inclusive scattering, shed light on the variation of flavour structure across the transverse profile of the proton. 
GPDs can also be accessed in the production of vector mesons [65]. Measurements of the $t$ and $W$ dependence of light $(\rho)$ and heavy $(J / \psi, \Upsilon)$ vector meson production will be important for the determination of proton structure and saturation effects at low $x$ at the LHeC.

\section{Electron-Nucleus Scattering}

\subsection{Nuclei}

Current knowledge of the partonic structure of nuclei is limited to a very small range of $x$ and $Q^{2}$ (Fig.18), and is likely to remain so for the foreseeable future now that there

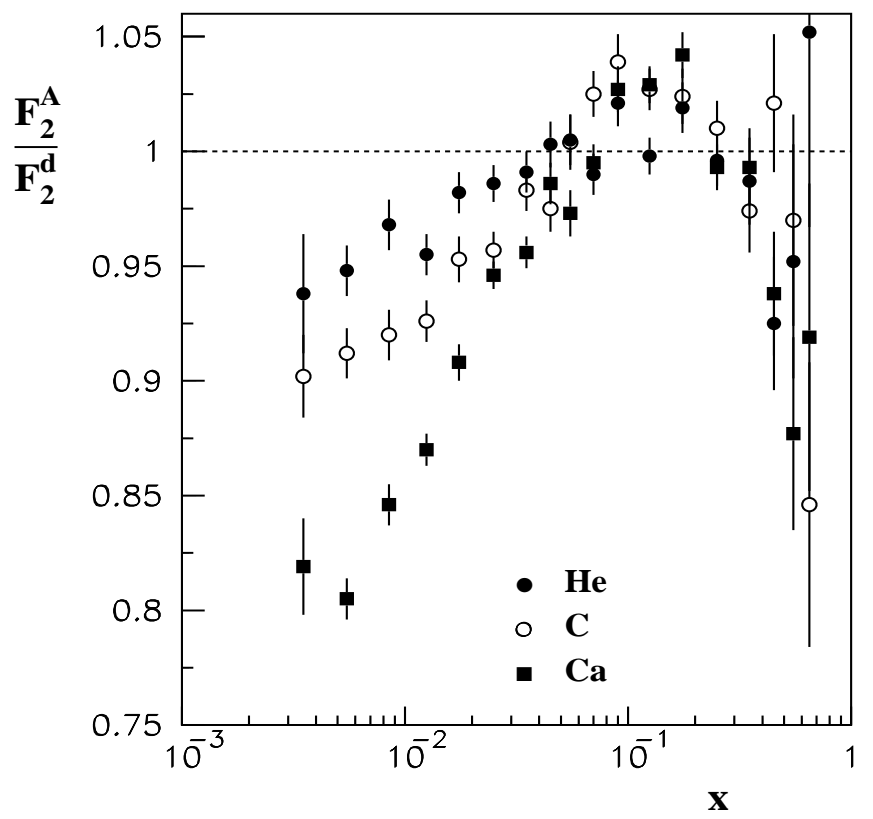

Figure 18: Ratio of $F_{2}$ structure functions in nuclei and the deuteron as measured in fixed target muon scattering by the NMC Collaboration. At the LHeC this measurement can be extended down to nearly $x=10^{-6}$ in the deep inelastic region. Note that the $Q^{2}$ values at the smallest $x \simeq 3 \cdot 10^{-3}$ in this plot are below $1 \mathrm{GeV}^{2}$ for the $\mathrm{NMC}$ but reach a few $1000 \mathrm{GeV}^{2}$ at the $\mathrm{LHeC}$.

are no plans to develop HERA as an electron-ion collider [66, 67]. The possibilities for $e A$ collisions in the future at lower energy $(\sqrt{s} \leq 100 \mathrm{GeV})$ at JLab [68] and at BNL [69, 70] will not access the low- $x$ region of nuclear structure, which is crucial to a full understanding of phase equilibria in nuclear matter, that is to the possible existence of a Quark-Gluon Plasma (QGP). Realisation of the LHeC will in contrast put in place a unique tool in the hunt for an understanding of chromodynamic phase equilibria.

The electron-nucleus scattering experiments at the LHeC will have a tremendous impact on the understanding of partonic matter in nuclei and on basic questions of 
QCD regarding the (de)confinement of quarks [71], the existence of a saturated gluon state, the Colour Glass Condensate [25], and the relationship of nuclear Gribov-Glauber shadowing to hard diffraction [72]. At a certain scale, due to unitarity, the rise of the gluon density towards low $x$ has to be tamed, and may not exceed a limit which is estimated to be roughly of the order of $Q^{2} / \alpha_{s}^{2}[9]$, with $Q^{2}$ in $\mathrm{GeV}^{2}$ and a factor of order 1 or smaller which depends on the dipole type considered in such estimates [73]. Such a limit, illustrated in Fig.19, may have been close to, but not yet reached, at HERA. The limit is likely to be observed in ep scattering at the LHeC and its effects in $e A$ scattering will be large, because the gluon density in nuclei is amplified $\propto A^{1 / 3}$.
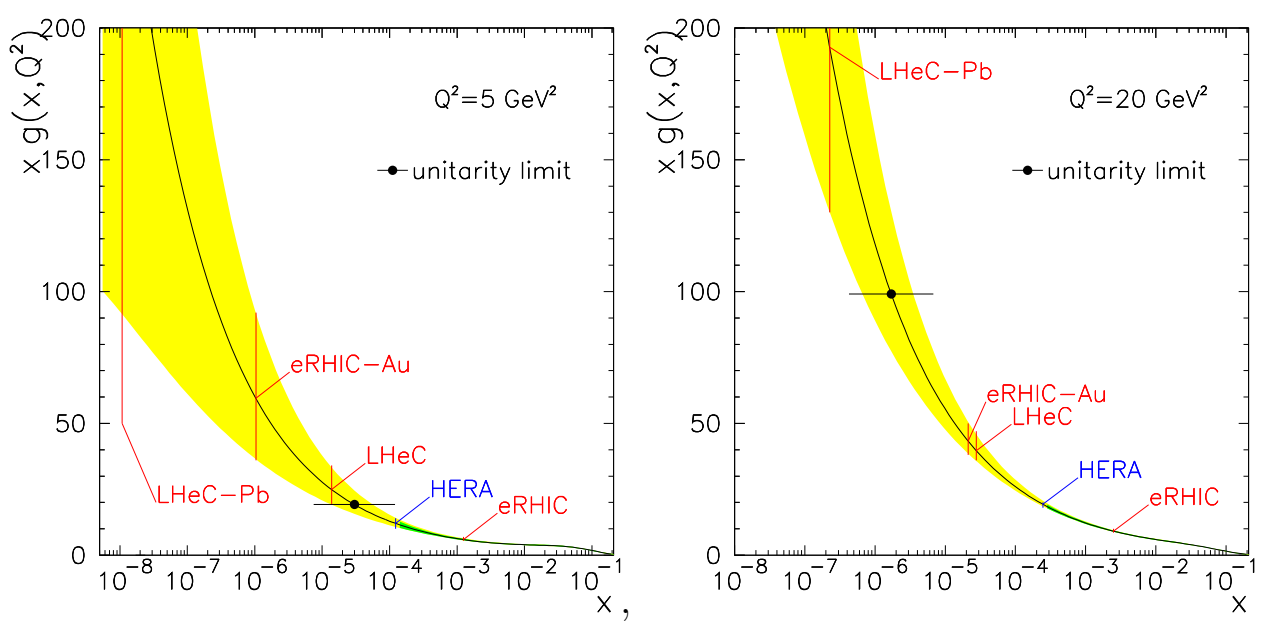

Figure 19: The gluon distribution as determined in an NLO DGLAP QCD analysis of $H 1$ data extrapolated from the accessed region above $10^{-4}$ to much lower values of Bjorken $x$, for $Q^{2}=5 \mathrm{GeV}^{2}$ (left) and $Q^{2}=20 \mathrm{GeV}^{2}$ (right). The gluon distribution is expected not to rise strongly beyond the unitarity limit, which is estimated to be of the order of $Q^{2} / \alpha_{s}\left(Q^{2}\right)$ (solid points). The extension of the kinematic range by the LHeC leads beyond the unitarity limit at lower $Q^{2}$ in ep scattering and also for larger $Q^{2}$ in eA scattering, when the increase of the gluon density in nuclei $\propto A^{1 / 3}$ is taken into account, considering here $\mathrm{ePb}$ scattering. Due to the much higher energy, the LHeC explores a region of much smaller $x$ than is accessible by eRHIC in eAu scattering. A clear observation of saturation in ep scattering at the LHeC will be crucial in distinguishing saturation phenomena from nuclear effects in $e A$ and $A A$ interactions.

In the regime of very high parton densities, the interaction of small colour singlets with hadronic targets becomes of comparable strength to the geometric cross section $\pi R_{A}^{2}$ and thus approaches the black disk limit or limit of opacity (absorption). In this limit of large, fixed $Q^{2}$ and decreasing $x$, striking observations are thereby expected in $e A$ DIS [74]:

- a change in the $Q^{2}, x$ dependence of the structure function, $F_{2} \rightarrow Q^{2} / \ln (1 / x)$,

- an increased diffractive scattering component approaching $50 \%$ of the scattering cross section and dominated by dijet production, and 
- a much less rapid decrease of the dependence of the cross sections on $Q^{2}$ for exclusive vector meson production.

The energy densities achieved in an $A A$ interaction at the LHC are immense, and to fully explore the nature of the interactions will require comparable data in $p A, p p$, and $e A$ collisions. LHeC and the LHC will thus constitute an experimental tool unparalleled in the history of hadron physics in that nowhere else has there ever been such a range of possible measurements at such an energy scale. Given the importance attached to an understanding of the existence and nature of a QGP, and given the complexities of this understanding, establishing $e A$ physics with the LHeC is of primary value.

\subsection{Deuterons}

Electron-deuteron scattering complements ep scattering in that it makes possible accurate measurements of neutron structure in the new kinematic range accessed by the LHeC. In a collider configuration, in which the hadron "target" has momentum much larger than the lepton probe, the spectator proton can be tagged and its momentum measured with high resolution [67]. The resulting neutron structure function data are then free of nuclear corrections. For the first time, since diffraction is related to shadowing, one will be able to control the shadowing corrections at the per cent level of accuracy [73].

Accurate en cross section measurements will resolve the quark flavour decomposition of the sea, i.e. via isospin symmetry, unfolding $\bar{u}$ from $\bar{d}$ contributions to the rise of $F_{2}^{p} \propto x(4 \bar{u}+\bar{d})$ towards low $x$, and, from the full set of $e^{ \pm} p$ and $e^{ \pm} n$ charged current cross section data, a full unfolding of the flavour content of the nucleon. For the study of the parton evolution with $Q^{2}$, the measurement of $F_{2}^{N}=\left(F_{2}^{p}+F_{2}^{n}\right) / 2$ is crucial [75] since it disentangles the evolution of the non-singlet and the singlet contributions. This provides additional accuracy in the determination of the strong coupling constant [76].

\section{Kinematics and Detector Requirements}

The kinematics of inclusive ep scattering are determined from the scattered electron with energy $E_{e}^{\prime}$ and polar angle $\theta_{e}$ and from the hadronic final state of energy $E_{h}$ and scattering angle $\theta_{h}$. The negative four-momentum transfer $Q^{2}$, the relative energy transfer $y$ and the Bjorken variable $x$ can be calculated from the scattered electron kinematics as

$$
\begin{aligned}
Q_{e}^{2} & =4 E_{e} E_{e}^{\prime} \cos ^{2}\left(\frac{\theta_{e}}{2}\right) \\
y_{e} & =1-\frac{E_{e}^{\prime}}{E_{e}} \sin ^{2}\left(\frac{\theta_{e}}{2}\right)
\end{aligned}
$$


and from the hadronic final state kinematics as

$$
\begin{aligned}
Q_{h}^{2} & =\frac{1}{1-y_{h}} \cdot E_{h}^{2} \sin ^{2}\left(\theta_{h}\right) \\
y_{h} & =\frac{E_{h}}{E_{e}} \sin ^{2}\left(\frac{\theta_{h}}{2}\right)
\end{aligned}
$$

where $x$ is given as $Q^{2} / s y$. Note that the angles $\theta$ are defined between the directions of the outgoing electron and the proton beam $\left(\theta_{e}\right)$ and between the hadronic final state and the proton beam $\left(\theta_{h}\right)$. The inclusive DIS cross section depends on two variables, besides the centre-of mass energy squared $s=4 E_{e} E_{p}=Q^{2} / x y$. The kinematic reconstruction in neutral current scattering therefore contains high redundancy, which is one reason why DIS experiments at ep colliders are precise 4 .

Following Eq,2, in charged current scattering the kinematics is reconstructed from the transverse and longitudinal momenta and energy of the final state particles according to 77

$$
\begin{aligned}
Q_{h}^{2} & =\frac{1}{1-y_{h}} \sum p_{t}^{2} \\
y_{h} & =\frac{1}{2 E_{e}} \sum\left(E-p_{z}\right) .
\end{aligned}
$$

The kinematics of ep scattering at the LHeC are illustrated in Fig.20. Lines of constant energy and angle of the scattered electron and the current jet are located in the $\left(Q^{2}, x\right)$ plane according to the relations:

$$
\begin{aligned}
& Q^{2}\left(x, E_{e}\right)=s x\left(1-E_{e}^{\prime} / E_{e}\right) /\left[1-x E_{p} / E_{e}\right] \\
& Q^{2}\left(x, E_{j}\right)=s x\left(1-E_{j} / x E_{p}\right) /\left[1-E_{e} /\left(x E_{p}\right)\right] \\
& Q^{2}\left(x, \theta_{e}\right)=s x /\left[1+x E_{p} \cot ^{2}\left(\theta_{e} / 2\right) / E_{e}\right] \\
& Q^{2}\left(x, \theta_{j}\right)=s x /\left[1+E_{e} \tan ^{2}\left(\theta_{j} / 2\right) / x E_{p}\right] .
\end{aligned}
$$

The accessible kinematic range is limited by the position and dimension of the focussing magnets, see Section 6.5. Limitation of the scattered electron angle to a value $\theta_{e, \min }$ defines, for not too small $x$, a constant minimum $Q^{2}$ which is independent of $E_{p}$ and which is a linear function of $E_{e}^{2}, Q^{2}\left(x, \theta_{e, \min }\right) \simeq\left[2 E_{e} \tan \left(\theta_{e} / 2\right)\right]^{2}$. Thus the LHeC, with a rather low electron beam energy of $70 \mathrm{GeV}$, allows the low $Q^{2}$ region to be accessed more easily than THERA [9]. As is illustrated in Fig.20, roughly a $1^{\circ}$ angular cut corresponds to a minimum $Q^{2}$ of about $1 \mathrm{GeV}^{2}$. To achieve high luminosity, focussing

\footnotetext{
${ }^{4} \mathrm{An}$ important example is the calibration of the electromagnetic energy scale from the measurements of the electron and the hadron scattering angles. This leads to energy calibration accuracies for $E_{e}^{\prime}$ at the per mil level at HERA, since in a large part of the phase space, around $x=E_{e} / E_{p}$, the scattered electron energy is approximately equal to the beam energy, $E_{e}^{\prime} \simeq E_{e}$, which causes a large "kinematic peak" in the scattered electron energy distribution. The hadronic energy scale can be obtained from the transverse momentum balance in neutral current scattering, $p_{t}^{e} \simeq p_{t}^{h}$. It is determined to around $1 \%$ at HERA.
} 


\section{LHeC - electron kinematics}

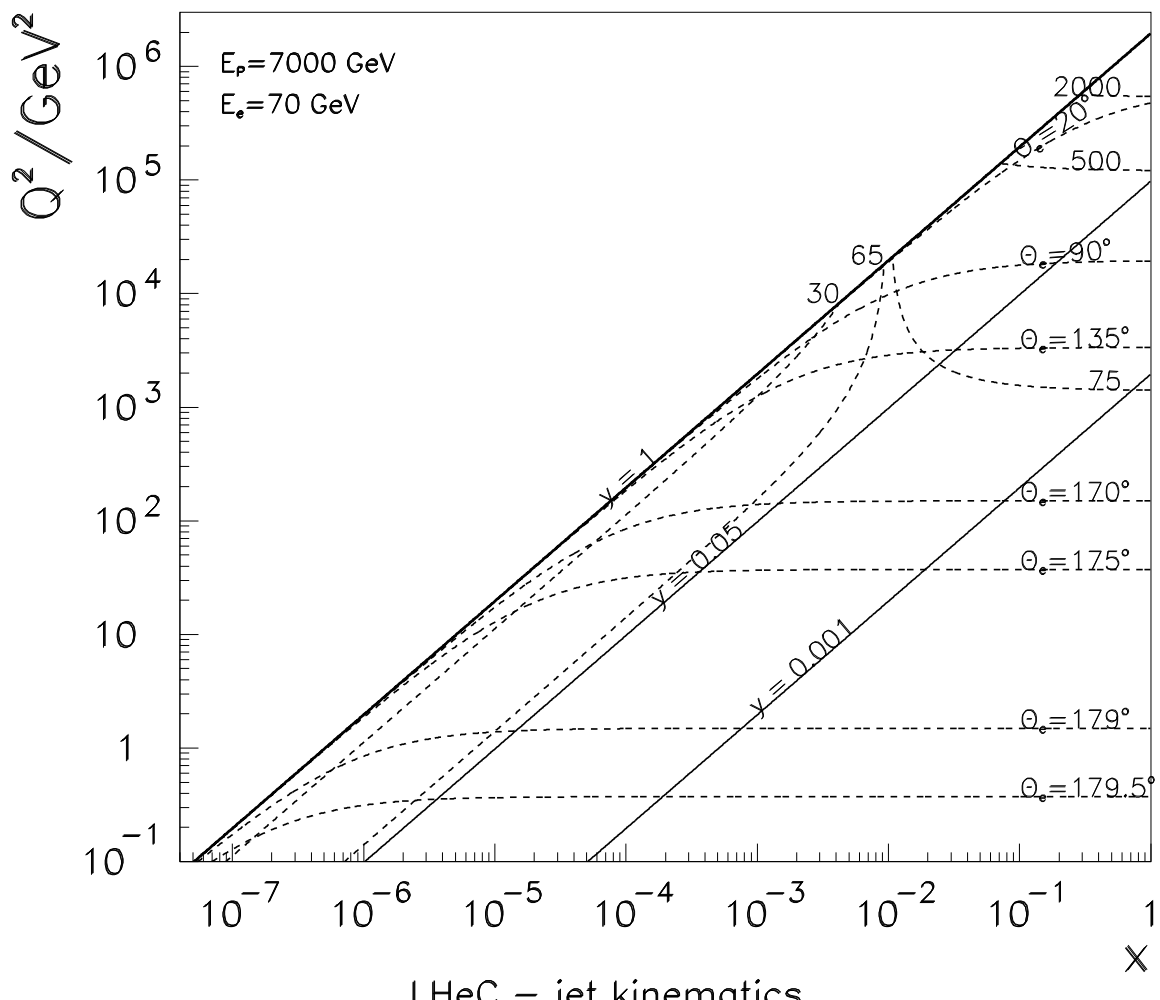

$\mathrm{LHeC}$ - jet kinematics

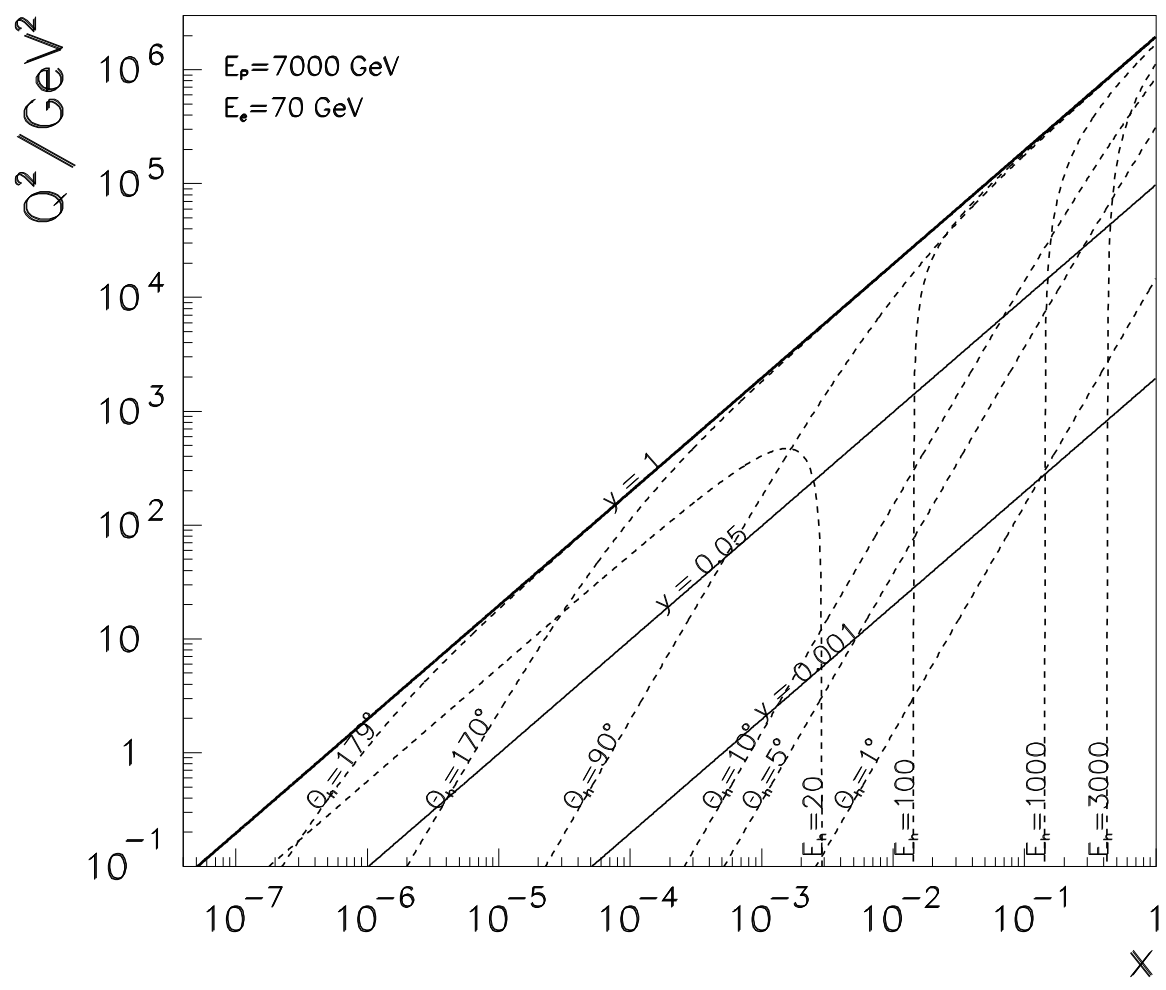

Figure 20: Kinematics of electron (top) and hadronic final state, "jet" (bottom) detection at the LHeC. 
magnets need to be placed close to the interaction region (IR). In the present design an aperture limit of $10^{\circ}$ is used. Thus for high luminosity operation, $Q^{2}$ reconstructed with electrons is larger than $100 \mathrm{GeV}^{2}$.

For THERA a "backward" electron spectrometer was sketched [9] using a calorimeter to reconstruct high energies of several hundreds of $\mathrm{GeV}$ and a silicon tracker telescope to measure the electron scattering angle down to $179.5^{\circ}$ and to identify the electron. The requirements at the $\mathrm{LHeC}$ are more modest, because $E_{e}^{\prime}$ is lower and the minimum $\theta_{e}$ is larger. At low $x$ the kinematics are such that the hadronic final state and the scattered electron energy add up to approximately the electron beam energy, $E_{e}^{\prime}+E_{h} \simeq E_{e}$. Final state physics at low $x$ requires access to the region within a few degrees of the beam pipe (Fig.201). Building a spectrometer for low $x$ physics with a $179(1)^{\circ}$ degree acceptance limit for $\theta_{e}\left(\theta_{h}\right)$ is challenging.

The increased asymmetry between the electron and proton beam energies at LHeC means that diffractively produced systems are strongly boosted in the outgoing proton direction. The rapidity gap-based diffractive selection, usually employed at HERA, will thus only be effective over a wide $x_{\mathbb{P}}$ range if there is substantial instrumentation at large rapidities (up to at least $\eta=5$ ). Another intriguing possibility is the use of Roman pots, which are planned for the LHC at the TOTEM experiment [78] and which are proposed in connection with ATLAS and CMS in the FP420 project [79].

At larger $Q^{2}$ the electron is scattered to angles between $170^{\circ}$ and $10^{\circ}$ with energies between about $10 \mathrm{GeV}$ and a few $\mathrm{TeV}$. A minimum angle cut $\theta_{h, \text { min }}$ in the forward region, the direction of the proton beam, excludes the large $x$ region from the hadronic final state acceptance (Fig. 20), along a line $Q^{2}\left(x, \theta_{h, \min }\right) \simeq\left[2 E_{p} x \tan ^{2}\left(\theta_{h, \min } / 2\right)\right]^{2}$, which is linear in the $\log Q^{2}, \log x$ plot and depends on $E_{p}$ only. Thus at $E_{p}=7 \mathrm{TeV}$ the minimum $Q^{2}$ is roughly $(1000[500] x)^{2}$ at a minimum angle of $10[5]^{\circ}$. Since this dependence is quadratic with $E_{p}$, lowering the proton beam energy may be of interest for reaching the highest possible $x$. As is seen in Fig. 20, the high luminosity IR $\left(\theta_{h}<\right.$ $170^{\circ}$ ) will have full coverage of the final state up to the largest $y$ for $Q^{2}$ above $100 \mathrm{GeV}^{2}$. The hadronic final state energies vary between a few $\mathrm{GeV}$ at low $x$ and a few $\mathrm{TeV}$ at large $x$. The central detector thus needs a strong solenoidal field, silicon and gaseous tracking detectors and a calorimeter capable of reconstructing electromagnetic and hadronic energy from a few tens of GeV up to a few TeV. Accurate muon identification and momentum measurement as needed for example for $b$ physics complement such an ep detector.

Issues on the interaction region (IR) and detector requirements were discussed previously at the LEP-LHC workshop [11, 7], which was held prior to the startup of HERA. The consideration of the machine-detector interface which is described in the next section is very preliminary. There has to be much further consideration on the detector and on the IR, with direct experience of the first operational LHC beam, of the optimisation for luminosity and physics scope than has been achieved at this stage. 


\section{Luminosity Prospects for the LHeC}

\subsection{Introduction}

In the following the possibilities are explored for achieving a high luminosity of leptonproton collisions using the existing LHC proton beam together with a new lepton storage ring in the LHC tunnel while maintaining the existing facility for proton-proton collisions. Earlier studies [80, 9] of possible lepton-proton colliders, which considered a configuration of a proton storage ring and a lepton linac, yielded a relatively small luminosity. Using the expressions derived in [80, 9] for the case of collisions between the LHC proton beam and a high energy lepton beam produced by a linear accelerator, the luminosity is given by

$$
L=4.8 \times 10^{30} \cdot \mathrm{cm}^{-2} \mathrm{~s}^{-1} \frac{N_{p}}{10^{11}} \cdot \frac{10^{-6} \mathrm{~m}}{\epsilon_{p N}} \cdot \frac{\gamma_{p}}{1066} \cdot \frac{10 \mathrm{~cm}}{\beta_{p}^{*}} \cdot \frac{P_{e}}{22.6 M W} \cdot \frac{250 \mathrm{GeV}}{E_{e}}
$$

Assuming the so-called "ultimate" LHC parameters [81] with the number of protons per bunch $N_{p}=1.67 \cdot 10^{11}$, the normalized proton transverse emittances of $\epsilon_{p N}=3.75$. $10^{-6} \mathrm{~m}$, a proton beta function $\beta_{p}^{*}$ of $0.5 \mathrm{~m}$ at the interaction point, a proton beam energy of $7 \mathrm{TeV}$, (corresponding to a Lorentz-factor of $\gamma_{p}=7460$ ) and assuming a lepton energy of $E_{e}=70 \mathrm{GeV}$ and an available electron beam power of $P_{e}=50 \mathrm{MW}$ one expects peak luminosities of $L=2.4 \cdot 10^{31} \mathrm{~cm}^{-2} \mathrm{~s}^{-1}$ for a ring-linac solution. The luminosity could be increased somewhat by reducing the proton emittance by using transverse cooling. The cooling of the bright and very high energy proton beams of the LHC is considered to be very challenging as experimental verification of proton beam cooling at these high energies has yet to be demonstrated. It thus seems worthwhile to reconsider the luminosity which could be achieved in a ring-ring configuration, using the LHC to accommodate an additional lepton storage ring, which would be installed above the existing superconducting magnets of the LHC and which would be brought into collisions with the LHC proton beam at one interaction point.

Similar estimates have been performed previously [10, 12]. The present estimate makes explicit use of the available experience of high luminosity operation at the circular lepton-proton collider HERA to derive a realistic scaling of the achievable luminosity of a ring-ring-based lepton proton collider in the LHC tunnel.

The aim of the present study is to investigate the parameters required for a realistic LHeC with a peak luminosity of $L=10^{33} \mathrm{~cm}^{-2} \mathrm{~s}^{-1}$ while maintaining the option for continued operations with proton-proton collisions.

The present study does not purport to be exhaustive in that detailed designs of the lattice, investigations of beam stability, and conceptual designs of accelerator components have not been performed. However, no limitations associated with these issues are foreseen as similar systems have been demonstrated in existing accelerators or will be demonstrated soon. In particular there is no detailed design of the magnets in the lepton-proton interaction region (IR) or the layout of other accelerator hardware systems. The injector system is assumed to be identical to the LEP injector system. 


\subsection{General Requirements}

The present study of the $\mathrm{LHeC}$ is based on the following general requirements and assumptions:

- A high luminosity of the order of $L=10^{33} \mathrm{~cm}^{-2} \mathrm{~s}^{-1}$ is assumed necessary to perform the physics programme in a reasonable amount of time.

- The electron beam energy should be as high as possible to obtain a large centre of mass energy which exceeds the HERA centre of mass energy of $318 \mathrm{GeV}$ by a significant factor. The choice of the centre of mass energy is motivated by the physics and is a sensitive parameter for the achievable luminosity. For this study values of around $E_{c m}=1.4 \mathrm{TeV}$ were explored corresponding to an electron beam energy of $70 \mathrm{GeV}$ given a proton beam energy of $7 \mathrm{TeV}$.

- It appears to be highly desirable to find a solution which allows for proton-proton collisions in the interaction regions (IR) of the LHC denoted by IR 1 and IR 5 during lepton proton collision operation. This implies that the proton beam parameters for LHeC are already predetermined by the design for the LHC. The parameters used in this study are listed in Tab.2 from [81].

- In order to provide sufficient space and acceptance for the experimental detector, a magnet-free space of at least $2.4 \mathrm{~m}$ is required in the interaction region. The aperture of the first accelerator lattice elements should allow for a detector acceptance angle of $10^{\circ}$.

- The electric power consumption of the $\mathrm{LHeC}$ should be in a reasonable range compared to the CERN overall power consumption.

Table 2: LHC proton beam parameters used in this study

\begin{tabular}{|l|c|c|}
\hline Proton Beam Energy & $\mathrm{TeV}$ & 7 \\
Circumference & $\mathrm{m}$ & 26658.883 \\
Number of Protons per bunch & $10^{11}$ & 1.67 \\
Normalized transverse emittance & $\mu \mathrm{m}$ & 3.75 \\
Bunch length & $\mathrm{cm}$ & 7.55 \\
Bunch spacing & $\mathrm{ns}$ & 25 \\
\hline
\end{tabular}

\subsection{Overall Parameters of the LHeC}

The luminosity of a high energy proton-lepton circular collider, expressed in terms of the limiting parameters, is given by

$$
L=\frac{I_{e} \cdot N_{p} \cdot \gamma_{p}}{4 \cdot \pi \cdot e \cdot \epsilon_{p N} \sqrt{\beta_{x p} \beta_{y p}}} .
$$


In this equation, $I_{e}$ is the total lepton beam current, $N_{p}$ is the number of protons per bunch, $\gamma_{p}$ is the Lorentz factor of the protons which depends on the proton beam energy, $\epsilon_{p N}$ is the normalized proton transverse beam emittance (which is assumed to be equal in both planes) and $\beta_{x p}$ and $\beta_{y p}$ are the values of the proton beam amplitude function ( $\beta$-function) at the interaction point (IP). Implicit in this formula are the requirements that the beam cross sections $\sigma$ of the proton and lepton beams at the IP be equal, $\sigma_{x p}=\sigma_{x e}, \sigma_{y e}=\sigma_{y e}$, which is consistent with experience at the SPS and HERA [82], and that the beam-beam tune shift parameters are in a tolerable range, which is particularly relevant for the lepton beam.

The proton beam parameters are assumed to be identical to the so-called "ultimate" LHC proton beam parameters with the beam energy of $7 \mathrm{TeV}$, the number of protons per bunch $N_{p}=1.67 \cdot 10^{11}$, and the normalized emittances of $\epsilon_{p N}=3.75 \cdot 10^{-6} \mathrm{~m}$. Given the aforementioned constraints, to achieve a luminosity of $L=1 \cdot 10^{33} \mathrm{~cm}^{-2} \mathrm{~s}^{-1}$, only the total lepton beam current $I_{e}$ and the beta-functions at the IP remain as free parameters.

The maximum lepton beam current in a high energy lepton storage ring is limited by the available RF power. Other potential limitations, such as the dissipation of this power around the accelerator or localized heating due to higher-order modes, can be avoided by an appropriate design of accelerator components. The total output $\mathrm{RF}$ power is typically limited by cost: an RF output power of $\mathrm{P}_{r f}=50 \mathrm{MW}$ (which is approximately $86 \%$ of the LEP power consumption or $28 \%$ of the 1999 CERN site power consumption of $910 \mathrm{GWh}$ [83] assuming a yearly operating time of $5000 \mathrm{~h}$ ) is considered here (somewhat arbitrarily) to be an upper limit. Since the basic ring parameters can only be varied slightly, the maximum lepton beam current is then determined by the lepton beam energy. A lepton which travels with an energy of $E_{e}$ around the ring with a bend radius of $\rho=2997 \mathrm{~m}$ incurs an energy loss per turn of $e \Delta U=C_{g} E_{e}^{4} /(e \rho)=$ $707 \mathrm{MeV}$ where $C_{g}=4 / 3 \pi r_{0} E_{0}^{-3}=8.821 \cdot 10^{-5} \mathrm{GeV}^{-3} \mathrm{~m}$ [84]. For a given available power, the total beam current is

$$
I_{e}=0.351 m A \cdot\left(P_{r f} / M W\right) \cdot\left(100 G e V / E_{e}\right)^{4},
$$

with $E_{e}=70 \mathrm{GeV}, P_{r f}=50 \mathrm{MeV}$ and assuming quasi loss-free superconducting $\mathrm{RF}$ resonators, the total beam current is $I_{e}=71 \mathrm{~mA}$.

With the proton beam parameters, the RF power and the lepton beam energy fixed, it remains to design the interaction region and a lepton accelerator lattice, which provides proton beta functions at the IP of at most $\beta_{x p} \beta_{y p}=1 \mathrm{~m}^{2}$, and which allows to match the cross section of the lepton beam while providing sufficient dynamic aperture. Since the electron beam has naturally unequal emittances $\epsilon_{x}, \epsilon_{y}$, whereas the proton beam has naturally equal emittances in the transverse planes, the ratio of the proton beta function at the IP $r_{\beta}$ is chosen close to four $\left(r_{\beta}=3.6\right)$ to ease matching the beam cross sections at the IP.

The lepton emittance determines the lepton beta functions at the IP since the beam cross sections need to be matched $\beta_{e} \epsilon_{e}=\beta_{p} \epsilon_{p}$. The choice of the lepton beam emittance is constrained to a window limited by dynamic and geometric aperture considerations, 
beam-beam tune shift limitations, by the so-called hourglass effect due to the long proton bunches (the rms proton bunch length is $\sigma_{p}=7.5 \mathrm{~cm}$ ), and by the necessity of small beam divergence at the IP to ease the beam separation and to avoid parasitic beam-beam effects.

The hourglass effect imposes a soft limit on the minimum beta function and the corresponding value of the emittance: $\beta_{x e} \gtrsim \sigma_{p} \rightarrow \epsilon_{x e} \lesssim \epsilon_{p N} \beta_{x p} /\left(\gamma_{p} \sigma_{p}\right)=13 \mathrm{~nm}$, $\beta_{y e} \gtrsim \sigma_{p} \rightarrow \epsilon_{y e} \lesssim \epsilon_{p N} \beta_{y p} /\left(\gamma_{p} \sigma_{p}\right)=3.8 \mathrm{~nm}$

The lepton emittance should further be sufficiently small to avoid a too large maximum beta-function in the low beta quadrupoles. The beta function at the IP, $\beta^{*}$, also determines the natural chromaticity contributions from the interaction region

$$
\xi_{I P}=\frac{1}{\pi} \cdot \frac{D_{e f f}}{\beta^{*}}
$$

where $D_{\text {eff }}$ is the effective distance of the low beta quadrupoles from the IP and the chromaticity contributions for the two transverse planes are assumed to be balanced. If the chromaticity contributions from the IR exceed a certain fraction $f$ of the contributions from the arc

$$
\xi_{\text {arc }}=\frac{N_{\text {cell }} \cdot \tan \left(\Phi_{\text {cell }} / 2\right)}{\pi}
$$

[85] the dynamic aperture might become a critical issue [86] according to experience at HERA, where the critical fraction is about $f=\xi_{I P} / \xi_{\text {arc }}=0.6$ [87] (c.f. LEP: $f=0.5$ [88]). Here $N_{\text {cell }}$ is the number of FODO cells and $\Phi_{\text {cell }}$ is the betatron phase advance per FODO cell.

With these considerations the limiting value of the horizontal lepton emittance $\epsilon_{x e}$ for matched beam cross sections is given by

$$
\xi_{I P} \leq f \xi_{\text {arc }} \rightarrow \epsilon_{x e, y e} \leq \frac{f N_{c e l l} \cdot \tan \left(\Phi_{\text {cell }} / 2\right) \cdot \beta_{x p, y p} \cdot \epsilon_{p N}}{D_{e f f} \gamma_{p}}
$$

We assume the fraction $f$ to be as large as the LEP value of 0.5 and assume that the chomaticity contributions from the non-interaction straights constitute $50 \%$ of the off-arc chromaticity contributions so that $f=0.25$. With a LEP-like lattice and $N_{\text {cell }}=290$, assuming an effective $D_{\text {eff }}=4 \mathrm{~m}$, one obtains for the emittances limiting values of $\epsilon_{x e}<23 \mathrm{~nm}, \epsilon_{y e}<5 \mathrm{~nm}$. As it turns out this small $\epsilon_{x e}$ value can just be achieved with the strongest focusing in a LEP-like structure with phase advances per FODO cell of $108^{\circ}$ and $90^{\circ}$ in the horizontal and vertical plane respectively [88].

The other limitation on the emittance of the lepton beam is due to the beam-beam effect which is suffered by the lepton beam and which is parameterised by the linear tune shift parameter

$$
\Delta \nu_{x, y}^{e}=\frac{\gamma_{p} r_{0} N_{p} \beta_{x e, y e}}{2 \pi \gamma_{e} \cdot \epsilon_{p N} \cdot \sqrt{\beta_{x p, y p}}\left(\sqrt{\beta_{x p}}+\sqrt{\beta_{y p}}\right)}=\frac{r_{0} N_{p} \sqrt{\beta_{x p, y p}}}{2 \pi \gamma_{e} \cdot \epsilon_{x e, y e}\left(\sqrt{\beta_{x p}}+\sqrt{\beta_{y p}}\right)}
$$

where $r_{0}$ is the classical electron radius. Tune shift parameters, which have been achieved at HERA, are $\Delta \nu_{y}^{H e}=0.05$ and $\Delta \nu_{x}^{H e}=0.03$ [90, 91]. The corresponding 
limit on the lepton beam emittances depends on the ratio of the proton beta functions at the IP, $r_{\beta}=\sqrt{\beta_{x p} / \beta_{y p}}$ :

$$
\begin{aligned}
\epsilon_{x e} & =\frac{r_{0} N_{p} \cdot r_{\beta}}{2 \pi \gamma_{e} \Delta \nu_{x}^{H e}\left(1+r_{\beta}\right)} \\
\epsilon_{y e} & =\frac{r_{0} N_{p}}{2 \pi \gamma_{e} \Delta \nu_{y}^{H e}\left(1+r_{\beta}\right)}
\end{aligned}
$$

For $r_{\beta}=1.9$ one obtains emittance limits of $\epsilon_{x e}=12 \mathrm{~nm}$ and $\epsilon_{y e}=4 \mathrm{~nm}$, respectively. However, since it will be difficult to separate the beams sufficiently for the short bunch spacing of 25ns, a larger horizontal beam-beam tune shift will be taken into account which allows a smaller value of the horizontal emittance. Based on these considerations, the horizontal emittance for the lepton accelerator in LHeC is chosen to be $\epsilon_{x e}=7.6 \mathrm{~nm}$ while the vertical emittance value is $3.8 \mathrm{~nm}$, assuming a coupling of $50 \%$. The choice of the ratio of proton beta function at the IP of $r_{\beta}=3.6$ implies lepton $\beta$-functions at the IP of $\beta_{x e}=12.7 \mathrm{~cm}$ and $\beta_{y e}=7 \mathrm{~cm}$. In order to achieve these emittances without too strong focusing, the number of FODO cells in the arc of LHeC is increased from the LEP value of 290 to 376.

The main parameters which are implied by the above considerations are listed in Tab.3.

Table 3: Main Parameters of the Lepton-Proton Collider

\begin{tabular}{|l|c|c|c|}
\hline Property & Unit & Leptons & Protons \\
\hline Beam Energies & $\mathrm{GeV}$ & 70 & 7000 \\
Total Beam Current & $\mathrm{mA}$ & 74 & 544 \\
Number of Particles / bunch & $10^{10}$ & 1.04 & 17.0 \\
Horizontal Beam Emittance & $\mathrm{nm}$ & 7.6 & 0.501 \\
Vertical Beam Emittance & $\mathrm{nm}$ & 3.8 & 0.501 \\
Horizontal $\beta$-functions at IP & $\mathrm{cm}$ & 12.7 & 180 \\
Vertical $\beta$-function at the IP & $\mathrm{cm}$ & 7.1 & 50 \\
Energy loss per turn & $\mathrm{GeV}$ & 0.707 & $6 \cdot 10^{-6}$ \\
Radiated Energy & $\mathrm{MW}$ & 50 & 0.003 \\
\hline Bunch frequency / bunch spacing & $\mathrm{MHz} / \mathrm{ns}$ & \multicolumn{2}{|c|}{$40 / 25$} \\
Center of Mass Energy & $\mathrm{GeV}$ & \multicolumn{2}{|c|}{1400} \\
Luminosity & $10^{33} \mathrm{~cm}^{-2} \mathrm{~s}^{-1}$ & \multicolumn{2}{|c|}{1.1} \\
\hline
\end{tabular}

\subsection{Lepton Ring}

A detailed design of an electron ring has not been carried out. The parameters of the lepton storage ring of the lepton proton collider are chosen to be as close as possible to those of LEP [89], which was housed in the LHC tunnel. 
The accelerator lattice consists of a FODO structure in the eight arcs with a betatron phase advance per cell of $\phi_{\text {cell }}=72^{\circ}$ in the horizontal and in the vertical plane. The cell length is $L_{\text {cell }}=60.3 \mathrm{~m}$ and the bending radius is $\rho=2997 \mathrm{~m}$. The ring has a circumference of $C=26658.876 \mathrm{~m}$. To further reduce the horizontal emittance, the damping distribution will be changed in addition to the strong focusing. The ring will be operated with a relative shift of the RF frequency of $\Delta f / f_{R F}=2.5 \cdot 10^{-7}$ which corresponds to an energy shift of $\Delta E / E=-1.8 \cdot 10^{-3}$. This changes the damping partition numbers in favour of horizontal damping to a value of $J_{x}=1.57$ and yields a beam emittance $\epsilon_{x e}$ of $7.6 \mathrm{~nm}$ at $70 \mathrm{GeV}$. The corresponding lepton beam energy spread is $(\delta E / E)_{R M S}=2.4 \cdot 10^{-3}$.

The beam current of $70.7 \mathrm{~mA}$ is composed of 2800 bunches spaced by $25 \mathrm{~ns}$ with $1.40 \cdot 10^{10}$ particles in each bunch.

With the bend radius of $2997 \mathrm{~m}$, a $70 \mathrm{GeV}$ electron (positron) will suffer an energy loss of $706.8 \mathrm{MeV}$ per turn by the emission of synchrotron radiation with a critical energy of $254 \mathrm{keV}$. The power loss of a beam of $70.7 \mathrm{~mA}$ amounts to $50 \mathrm{MW}$. The linear power load to the vacuum system is $27 \mathrm{~W} / \mathrm{cm}$. This is a large but feasible value. It exceeds the values of HERA (5.2 MW dissipated over $3818 \mathrm{~m}$ yielding $13.5 \mathrm{~W} / \mathrm{cm}$ ) by about a factor of two. The cooling system used at HERA is both conservative and conventional. The values for LEP were $9 \mathrm{~W} / \mathrm{cm}$ with a critical energy of $522 \mathrm{keV}$ for a beam energy of $100 \mathrm{GeV}$ and a beam current of $12 \mathrm{~mA}$ [92].

The RF system is assumed to consist of superconducting $1 \mathrm{GHz}$ RF resonators with an accelerating gradient of $12 \mathrm{MV} / \mathrm{m}$. An active RF structure length of $127 \mathrm{~m}$ or 845 resonator cells is required to produce a total voltage of $1521 \mathrm{MV}$. This voltage allows a synchronous phase of $28^{\circ}$ and provides an RF bucket which accommodates ten times the RMS beam energy spread. This is expected to provide sufficient margin for a good beam lifetime. The parameters of the lepton ring are summarized in Tab4.

Proven superconducting RF technology for lepton storage rings exists for only half the envisioned RF gradient 93]. However, design efforts for superconducting RF for continuous operation $(\mathrm{CW})$, for large beam currents and high bunch intensities are underway for ERL and CW-LINAC applications (see for example [94]). The corresponding designs could serve as a starting point for an appropriate layout of a LHeC $\mathrm{RF}$ system. More development is required in the design of input couplers and higher order mode couplers.

Geometrical considerations need to be taken into account in accommodating an additional lepton ring in the existing LHC tunnel. In the arc sections there appears to be sufficient space to place the lepton beam line above the LHC magnets. In the straight section, it may be cumbersome to accommodate the large RF systems of the lepton ring.

Since the ATLAS and CMS detectors are assumed to remain active at their locations when the lepton-proton collider is operated, a bypass must be provided round them. There exist survey tunnels which are parallel with the LHC straight sections 1 and 5 which could be used for a bypass of the caverns which house the experimental detectors. 


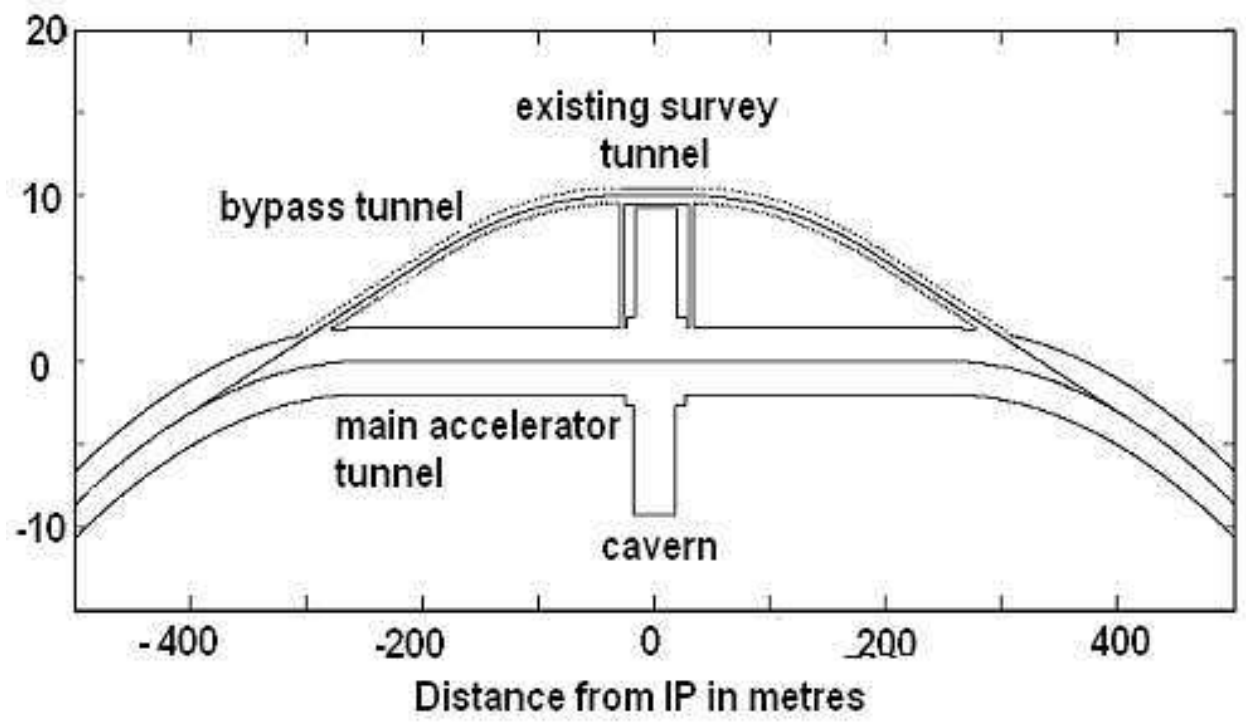

Figure 21: Top view (schematically) of straight section around IP1 (IP5) with an e-ring bypass around the experimental caverns of ATLAS and CMS. The scales are in meters.

They have a distance of about $10 \mathrm{~m}$ from the LHC beam axis and a length of about $100 \mathrm{~m}$. Two about $250 \mathrm{~m}$ long, up to $2 \mathrm{~m}$ diameter connection tunnels would have to be drilled from the end of the arcs to connect to these tunnels (see sketch in Fig.211).

Lepton beam instabilities are not expected to become an important performance limitation given that the design single bunch currents are relatively modest. The expected total impedance, roughly estimated, is less than the impedance of LEP. The bunch population is much lower than that of LEP. For this reason, there should be no single bunch beam current limitations. A conventional active damper system could be used to damp coupled bunch oscillations if needed.

An open issue concerns the dynamic aperture of the lepton ring taking into account the contributions of the chromaticity in the interaction region. In this design study the beam emittance has been derived using scaling laws. Dynamic aperture studies will be necessary to assure sufficient stability.

\subsection{Interaction Region}

The electron-proton interaction region is taken to be installed in one of the existing LHC straight sections. The main detectors CMS and ATLAS are assumed to remain active during the operation of the lepton proton collider thus excluding the possibility of lepton-proton collisions in the straight sections around IP5 and IP1. The straight sections around IP7 and IP3 are needed for beam cleaning, which cannot be compromised for LHC proton operation. IP4 and IP6 are occupied by the proton RF and beam dump systems. This leaves only IP2 which is occupied by the ALICE detector and IP8 which is occupied by LHCb. A lepton-proton physics programme at the LHC 
Table 4: Parameters of the electron ring accelerator

\begin{tabular}{|l|l|l|}
\hline Electron Ring Parameters & Unit & Value \\
\hline Parameter & $\mathrm{m}$ & 26658.86 \\
\hline Circumference C & $\mathrm{GeV}$ & 70 \\
\hline Beam Energy $\mathrm{E}_{e}$ & & FODO \\
\hline Arc Focusing & $\mathrm{m}$ & 60.3 \\
\hline Cell length $\mathrm{L}_{c}$ & $\mathrm{~m}$ & 2997 \\
\hline Bending radius $\rho$ & degree & 72 \\
\hline Horizontal betatron Phase Adv./cell $\Delta \phi_{x}$ & degree & 72 \\
\hline Vertical betatron Phase Adv./cell $\Delta \phi_{y}$ & & 376 \\
\hline Number of FODO cells in the Arcs $\mathrm{N}_{\text {cell }}$ & & $94 / 120$ \\
\hline Arc Chromaticity (hor/vert.) $\xi_{x, y}$ & $\mathrm{~mA}$ & 70.7 \\
\hline Beam Current $\mathrm{I}_{e}$ & $\mathrm{~ns}$ & 25 \\
\hline Bunch spacing $\tau_{b}$ & & 2800 \\
\hline Number of bunches $\mathrm{n}_{b}$ & $10^{10}$ & 1.4 \\
\hline Number of particles per bunch $\mathrm{N}_{e}$ & $10^{-4}$ & 1.34 \\
\hline Momentum compaction factor $\alpha$ & $\mathrm{nm}$ & 7.6 \\
\hline Horizontal beam emittance $\epsilon_{x e}$ & $\mathrm{~nm}$ & 3.8 \\
\hline Vertical beam emittance $\epsilon_{y e}$ & $10^{-3}$ & 2.4 \\
\hline RMS energy spread $\sigma_{e}$ & $\mathrm{~mm}$ & 7.1 \\
\hline RMS bunch length & $\mathrm{MeV} /$ turn & 706.8 \\
\hline Particle Radiation energy loss per turn $\mathrm{e} \mathrm{U}_{\text {loss }}$ & $\mathrm{MW}$ & 50 \\
\hline Beam Power loss $\mathrm{P}_{\text {loss }}$ & $\mathrm{MV}$ & 1521 \\
\hline Circumferencial Voltage U & $\mathrm{degree}$ & 27 \\
\hline Synchronous Phase $\phi_{s y n c h}$ & $\mathrm{MHz}$ & 1000 \\
\hline RF frequency $\mathrm{f}_{r f}$ & $\sigma_{e}$ & 8.4 \\
\hline Bucket height $\mathrm{h}_{b}$ & $\mathrm{~Hz}$ & 250 \\
\hline RF frequency shift & $\mathrm{f}_{r e v}$ & 0.191 \\
\hline Synchrotron frequency $\mathrm{f}_{s}$ & & \\
\hline
\end{tabular}




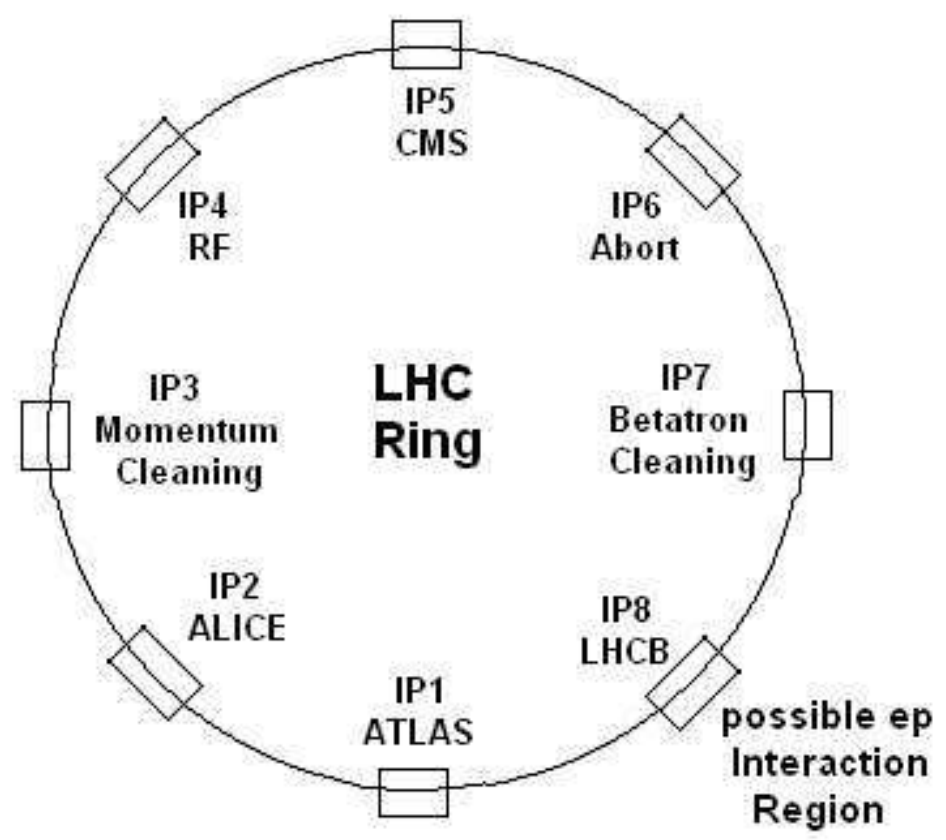

Figure 22: Possible location for the lepton-proton interaction region assuming that the $B$ physics programme is finished when the LHeC is installed.

would be unlikely to begin before the $B$-physics programme is completed. In this case one may envisage an interaction region for the LHeC around IP8 (Fig.22).

In order to provide small beta, the low beta quadrupole magnets have to be reasonably close to the IP. This requires a quick separation of the two beams outside the collision region. Separation by strong magnetic fields produces high power synchrotron radiation which is problematic because of experimental backgrounds and heating of the vacuum system. The alternative, a large crossing angle, reduces the luminosity. The IR design has to compromise between these difficulties.

In order to allow for simultaneous lepton-proton and proton-proton operation, the following collision scheme is proposed. The lepton bunch spacing is $25 \mathrm{~ns}$, which is the nominal LHC proton bunch spacing. The two proton beams traverse the low beta quadrupoles of the lepton beam and the IP with a relative angle of $\simeq 6 \mathrm{mrad}$ in the horizontal plane. The non-colliding proton bunch is vertically displaced, thus providing a vertical separation to the lepton and colliding proton beam. The lepton beam passes the IP with an angle of $\theta_{c}=2 \mathrm{mrad}$ in the horizontal plane relative to the colliding proton beam.

The interaction region design is thus based on a moderate horizontal crossing angle of $2 \mathrm{mrad}$. This implies that the interaction geometry is anti-symmetric around the interaction point which leads to a narrower synchrotron radiation fan. This helps with the collimation scheme. The crossing angle avoids large synchrotron radiation power dissipation in the cold beam pipes of the proton low-beta quadrupoles. To regain the associated luminosity reduction by a factor of 3.5 caused by the crossing angle, socalled crab-crossing is assumed, which requires a tilt of the proton bunches around a vertical axis. This is discussed further below. 
At the first parasitic electron-proton collision point at $3.72 \mathrm{~m}$ from the IP, the lepton and the proton beam are separated by about $7.8 \mathrm{~mm}$ or $8.4 \sigma$ of the horizontal lepton beam size. This separation is considered sufficient to avoid potentially harmful interactions due to so-called parasitic crossings.

The length of the magnet-free space for the detector beam-pipe is assumed to be $\pm 1.2 \mathrm{~m}$. The space requirement for the first low beta quadrupole of the lepton beam is $400 \mathrm{~mm}$ in diameter. This provides a detector acceptance angle of $9.4^{\circ}$ around the longitudinal axis.

The low beta quadrupole magnets for both protons and leptons are assumed to be superconducting. The focusing of the electron beam could be accomplished using a low-beta quadrupole triplet located at a distance of $1.2 \mathrm{~m}$ from the IP followed by an $50 \mathrm{~m}$ long drift without focusing elements. The superconducting low beta quadrupole magnets have a gradient of $93 \mathrm{~T} / \mathrm{m}$, lengths of $96 \mathrm{~cm}, 204 \mathrm{~cm}$ and $114 \mathrm{~cm}$, respectively, and they provide half apertures of $30 \mathrm{~mm}, 40 \mathrm{~mm}$ and $55 \mathrm{~mm}$.

The beam separation is accomplished as follows. The low beta quadruples are displaced by $0.25 \mathrm{~mm}$ from the beam axis which constitutes a $4.15 \mathrm{~m}$ long soft separating magnetic field which provides a deflection of $0.4 \mathrm{mrad}$. The low beta triplet is followed by a long soft separator dipole magnet with a field of only $0.023 \mathrm{~T}$ and a length of $15 \mathrm{~m}$. The separation provided by this magnet is $1.5 \mathrm{mrad}$. This arrangement, together with the crossing angle of $2 \mathrm{mrad}$ provides a beam separation of $62 \mathrm{~mm}$ at $22 \mathrm{~m}$, where the first low beta magnet for the proton can be located.

These magnets can be built using standard superconducting and normal conducting magnet technology. The larger aperture of the third quadrupole magnet is needed to provide sufficient aperture for the second, non-colliding, proton beam.

The colliding proton beam passes off-centre through the lepton low beta triplet before entering the first magnet of the proton low-beta triplet at $22 \mathrm{~m}$. The first magnet must ensure an integrated strength of $1564 \mathrm{~T}$, the second lens requires $2070 \mathrm{~T}$ and the third lens has an integrated gradient of $1058 \mathrm{~T}$. The total length is about $45 \mathrm{~m}$. Gradients of the order of $115 \mathrm{~T} / \mathrm{m}$ are required and an aperture of $15 \mathrm{~mm}$ is needed. All quadrupole magnets are assumed to have a cold beam pipe and cold iron to provide flux return.

The first of these magnets is a septum half quadrupole as in the case of the HERA interaction region. The width of the septum is $12 \mathrm{~mm}$. The pole tip field is $2.79 \mathrm{~T}$. The mirror-yoke is made of high quality magnetic steel. The large return yoke of the quadrupole magnets of the second proton low-beta lens accommodates a lepton beam pipe at room temperature which is to be cooled. The separation at the third group of magnets of the proton low beta triplet is sufficiently large, for the lepton to pass outside the cryostat.

After the low-beta triplet of the proton beam, the lepton beam is deflected by $5 \mathrm{mrad}$ vertically using two $10 \mathrm{~m}$ long dipoles which provide kicks of $1 \mathrm{mrad}$ and $4 \mathrm{mrad}$ respectively. At the end of the straight section, the lepton beam is about $80 \mathrm{~cm}$ above 
the proton beam axis. The proton beam orbits diverge to $80 \mathrm{~cm}$ separation. After the vertical deflection of the lepton beam, the protons are matched to their arc trajectory with three ten meter long superconducting dipole magnets.

The non-colliding proton beam is assumed to cross the colliding proton beam at the IP with an angle of $6 \mathrm{mrad}$ and a sufficient vertical separation. It is assumed to bypass the proton low-beta triplet. No attempts have been made to produce a layout of the lattice for this beam in the IR because it seems non-problematic.

This arrangement allows for a beta function of the lepton beam at the IP of $\beta_{x e}=12 \mathrm{~cm}$ and $\beta_{y e}=7 \mathrm{~cm}$. The peak values of the vertical and horizontal lepton beta functions amount to $906 \mathrm{~m}$ and $269 \mathrm{~m}$. The critical chromaticity contributions from the IR are quite modest with values of $\xi_{x I R}=-7$ and $\xi_{y I R}=-39$, which is about $21 \%$ of the contributions of the arc. Correction of this chromaticity is not expected to result in any significant reduction of dynamic aperture. The horizontal and vertical peak beta functions of the proton beam are $2668 \mathrm{~m}$ and $2637 \mathrm{~m}$, respectively, and so are considerably smaller than at the proton-proton interaction points and are assumed to be non-problematic. The IR chromaticity contributions of the proton beam are $\xi_{\text {pir }}=$ -8 .

The low beta quadrupole lenses provide sufficient aperture of at least 13.5 times the RMS beam size in the case of protons and at least 20 times the RMS beam size in the case of leptons. According to experience at HERA [90], this would be sufficient for avoiding beam lifetime reductions or poor backgrounds.

The interaction region is sketched in Fig.23 and Fig.24. The lattice functions are plotted in Fig.25. The interaction region parameters are shown in Table 5.

The crossing angle of $\theta_{c}=2 \mathrm{mrad}$ reduces the luminosity by a factor of 3.5 . One can recover from this reduction and can avoid any detrimental effects from the finite crossing angle if the proton beam is tilted around a vertical axis by $\theta_{c} / 2$. This can be accomplished by RF resonators with a transverse deflecting field, so-called crab cavities. In order to obtain an almost linear kick with the distance from the centre of the proton bunch over the entire length of the bunch $\left(\sigma_{p}=75 \mathrm{~mm}\right.$ is the RMS proton bunch length), the RF wave length has to be much larger than the bunch length. An $\mathrm{RF}$ frequency of $500 \mathrm{MHz}$ fulfils this requirement (the non-linearity at $\sigma_{p}$ from the centre amounts to $9 \%$ ). With the beta function of $708 \mathrm{~m}$ at the properly placed crab cavities, the required transverse kick at $\sigma_{p}$ from the centre is $\kappa=2.1 \mu \mathrm{rad}$. The $\mathrm{RF}$ phase angle corresponding to one $\sigma_{p}$ is $42^{\circ}$. This means that the RF structure has to provide an integrated peak field of $U_{c r a b}=\kappa E_{p}=20.8 \mathrm{MV}$. With a gradient of $G_{\text {crab }}=3.4 \mathrm{MV} / \mathrm{m}$, the two crab cavity systems around the IP must have an active length of $6.1 \mathrm{~m}$ each. This could be accomplished by a three 6 -cell resonators. A 12 $m$ long superconducting $500 \mathrm{MHz}$ RF structure is not expected to affect the LHC impedance budget considerably if the higher order modes are damped below a $\mathrm{Q}_{H O M}$ of 1000. Shorting of the resonators during p-injection and ramping may be required.

The two crab cavities can be installed in the IR at the point of maximum separation of the proton orbits between $120 \mathrm{~m}$ and $140 \mathrm{~m}$ distance from the IR. The horizontal 


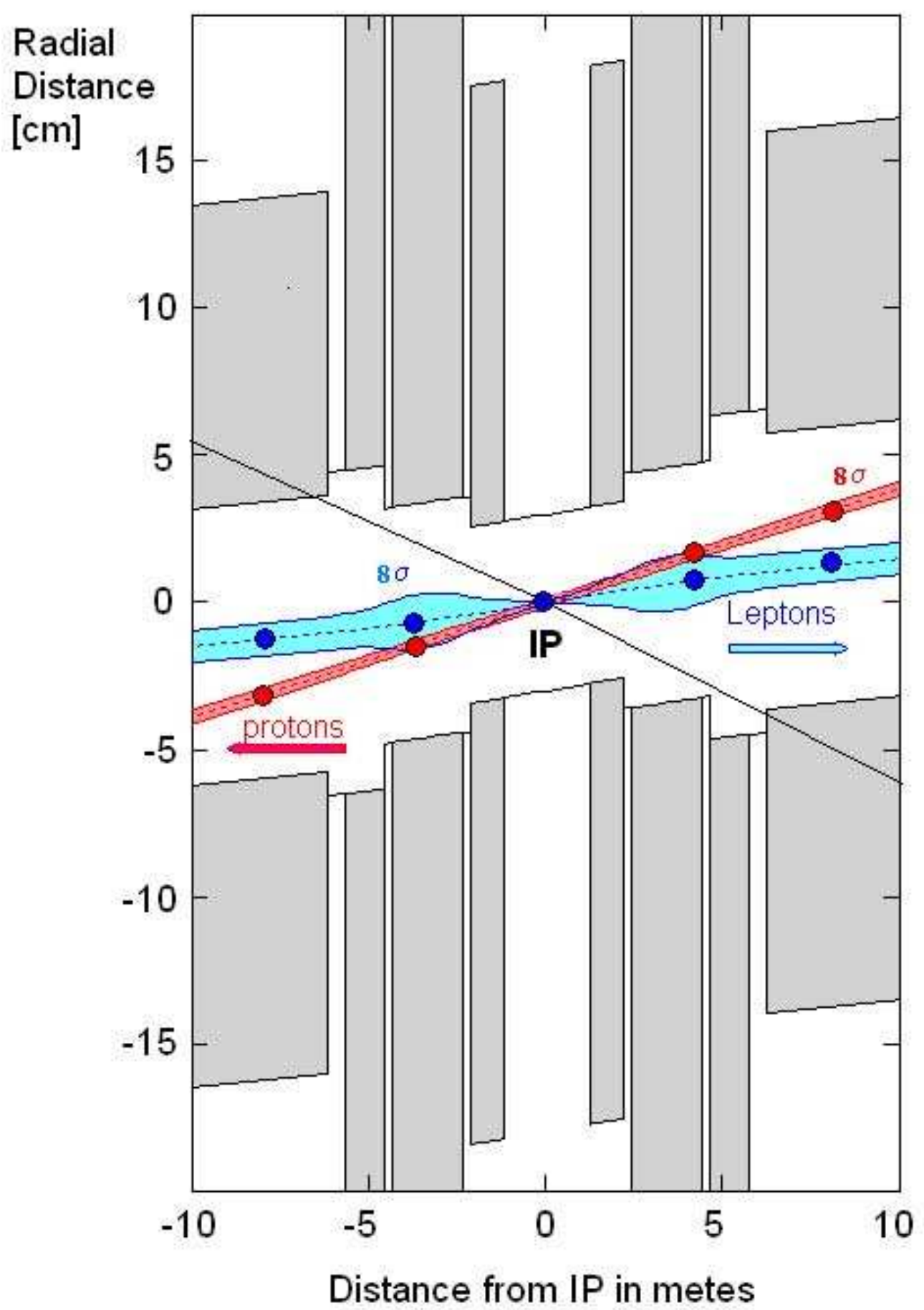

Figure 23: Top view (schematically) around the IP. The $8 \sigma$ beam envelope of the proton and the lepton beams are shown. The non-colliding proton beam (dashed line) envelope is not shown. 


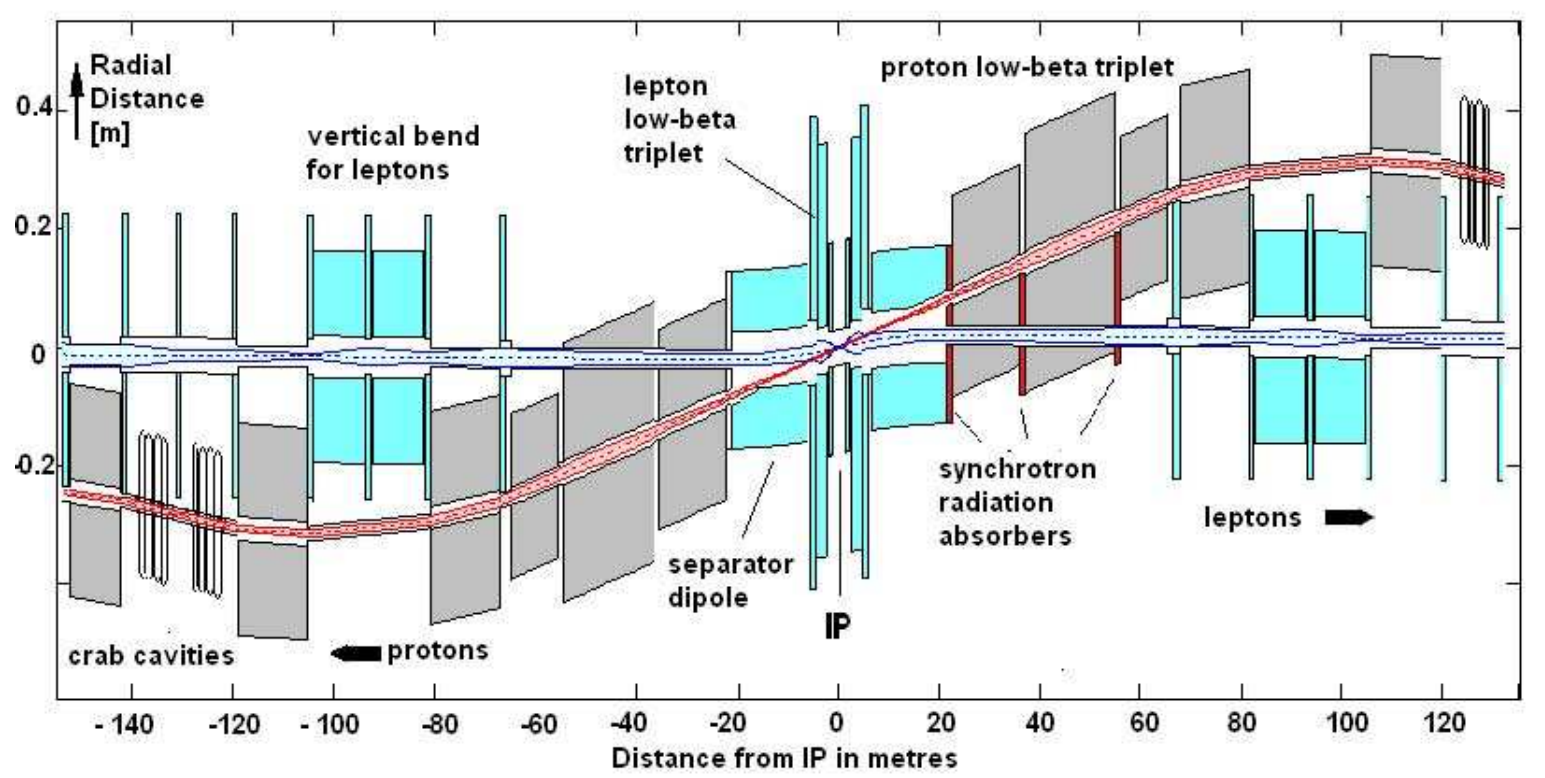

Figure 24: IR conceptional overview (top view)

phase advance at $120 \mathrm{~m}$ from the IP amounts to $25.6^{\circ}$. Thus a third resonator may be needed to provide exact closure of the crab-orbit bump. In order to avoid blow-up of the proton beam, the tolerances on the RF system and the required precision of the field amplitudes in the presence of high beam loading are very demanding. No attempt has been undertaken so far to specify an RF design for the crab-system.

The horizontal amplitude of the 'crabbed' trajectories amounts to $0.3 \mathrm{~mm}$ for particles which are one standard deviation away from the centre. The corresponding increase in aperture requirement is negligible.

For the low $x$, low $Q^{2}$ part of the physics programme, the detector needs acceptance to polar angles of $1^{\circ}$ with respect to the detector axis. This cannot be provided by the high luminosity IR as presented here. Since this part of the physics programme needs substantially less luminosity ( $\sim$ one order of magnitude), the low beta magnets can be placed further away from the IP (by $3 \mathrm{~m}$ ). The beta function at the IP the increases by a factor of 4 and a larger crossing angle can be tolerated. The low beta quadrupole triplet for the lepton beam needs a larger aperture to accommodate all the beams. No further effort has yet been made with the low $x$ IR configuration, which is not expected to represent a design challenge.

\subsection{Synchrotron Radiation}

The synchrotron radiation produced by the beam separation magnets, by misalignment of the quadrupole magnets, and by closed orbit distortions in the IR, must be minimized by using beam-based alignment, by careful orbit control and by an effective collimation system. These issues have been discussed in the preparation and commissioning of the 


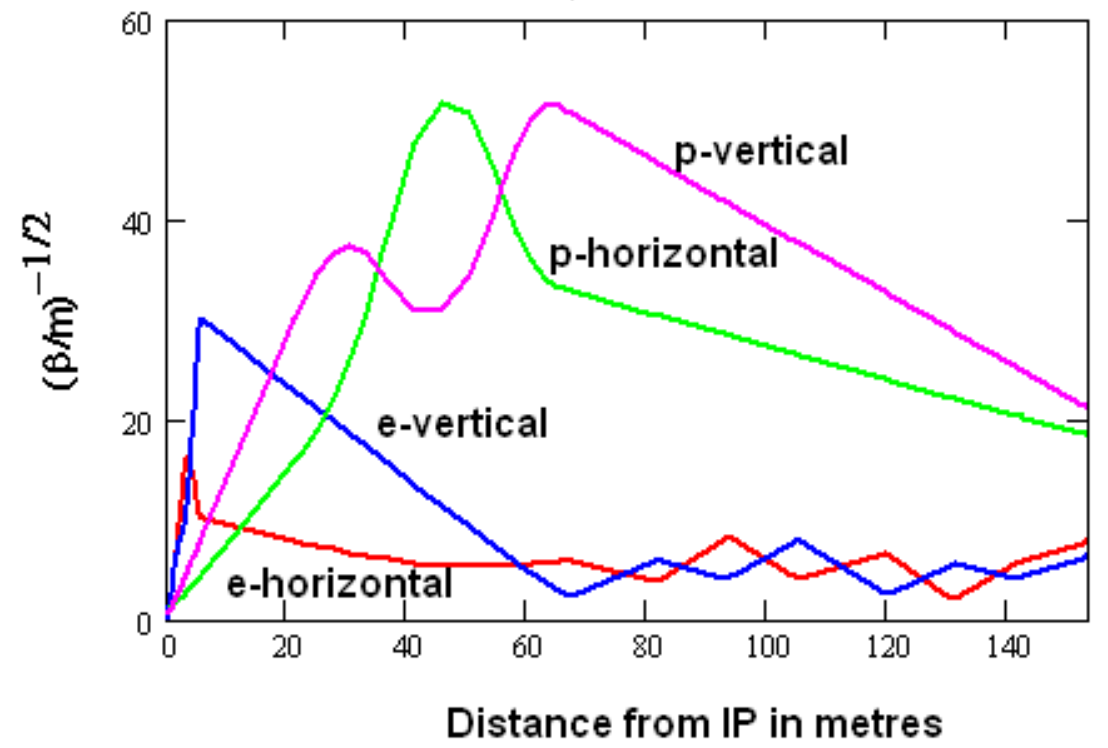

Figure 25: Lattice functions for the proton and lepton beam in the lepton-proton IR.

HERA luminosity upgrade [95, 96. The details will not be discussed here but it is important to notice that the synchrotron radiation produced in the proposed IR is somewhat less critical than in the case of HERA.

The soft bending field with a bend radius of $\rho_{i r}=10,010 \mathrm{~m}$ produces a radiation of $9.1 \mathrm{~kW}$ and a critical energy of $76 \mathrm{keV}$, which is transported within a fan of $1.9 \mathrm{mrad}$ horizontal opening angle and a vertical RMS thickness of $\approx 2 \mathrm{~mm}$. The narrow opening angle of the fan is due to the anti-symmetric arrangement of the IR. Due to the crossing angle in the IP, the whole synchrotron radiation fan is tilted by $2 \mathrm{mrad}$ away from the proton beam. Therefore, the high power synchrotron radiation does not penetrate the cold low-beta quadrupole magnets of the proton beam. Fig.[26] shows the distribution of synchrotron radiation in the IR.

The radiation is stopped at the $1 \mathrm{~m}$ long absorber placed at $21 \mathrm{~m}$ from the IP before the first proton low-beta quadrupole. The linear power density reaches maximum values of $4.0 \mathrm{~kW} / \mathrm{cm}$ (Fig.27). The absorbing surface is tilted by $(\pi / 2-5 \mathrm{mrad})$ so that the surface power density is reduced to approximately $100 \mathrm{~W} / \mathrm{mm}^{2}$. A corresponding collimator exists at HERA [96, 98]. It absorbs about 3 to $4 \mathrm{~kW}$ of radiation with a power density of $40 \mathrm{~W} / \mathrm{mm}^{2}$. The absorber presented here (Fig. 28) is a realistic extrapolation of the HERA absorber. A sketch of a possible absorber for LHeC is shown in Fig.28. A second absorber is needed between the first and the second low beta quadrupoles of the proton beam optics. According to HERA experience [97] this collimation system should be adequate for both elimination of experimental background and protection of accelerator components. 
Table 5: Parameters of the high luminosity interaction region for the $\mathrm{LHeC}$

\begin{tabular}{|l|l|l|l|}
\hline Interaction region parameters & unit & leptons & protons \\
\hline property & $\mathrm{cm}$ & 12.7 & 180 \\
\hline Horizontal Beta function at IP & $\mathrm{cm}$ & 7.07 & 50 \\
\hline Vertical beta function at IP & & -7.5 & -7.9 \\
\hline Horizontal IR Chromaticity & & -29.7 & -7.7 \\
\hline Vertical IR chromaticity & $\mathrm{m}$ & 131.7 & 2279 \\
\hline Maximum horizontal Beta & $\mathrm{m}$ & 704.4 & 2161 \\
\hline Maximum vertical Beta & $\sigma_{x}$ & 16 & 13.5 \\
\hline Minimum of available Aperture & $\mathrm{T} / \mathrm{m}$ & 93.3 & 115 \\
\hline Low beta quadrupole gradient & $\mathrm{T}$ & 0.033 & - \\
\hline Separation dipole field & $\mathrm{kW}$ & 9.1 & - \\
\hline Sychrotron Radiation Power & $\mathrm{m}$ & $.96 / 2.43 / 1.14$ & $16.5 / 18.6 / 11$ \\
\hline Low beta quadrupole length & $\mathrm{mm}$ & $30 / 40 / 50$ & $12 / 15 / 15$ \\
\hline Low beta quadrupole apertures & $\mathrm{m}$ & 1.2 & 22 \\
\hline Distance of first quadruple from IP & $\mathrm{degree}$ & & 9.4 \\
\hline Detector Acceptance Polar Angle & $\mathrm{mrad}$ & & 2 \\
\hline Crossing Angle & & & \\
\hline
\end{tabular}

\subsection{Magnetic Elements of the Interaction Region}

The superconducting low beta quadrupoles for the electron beam can be built using standard superconducting technology. The first two low beta quadrupoles for the protons are more challenging, because the lepton beam pipe has to pass through the cryostat of these magnets.

The first lens is a septum quadrupole laid out as a superconducting half quadrupole. The radius of the half aperture is $30 \mathrm{~mm}$ which provides a $15 \mathrm{~mm}$ aperture for the beam. The left side half of the magnets looks like a standard superconducting quadrupole (Fig.29). The other half consists of magnetic iron with a gap for the lepton beam. The achieved gradient is $93 \mathrm{~T} / \mathrm{m}$. The magnetic mirror plate works well up to a magnetic induction of $2.79 \mathrm{~T}$ near the coil. The magnet has a reasonable field quality and has no field in the gap for the lepton beam. This has been confirmed by magnetic field calculation using the code Opera-2D [99]. Figures 29, 30 and 31 show a cross section with the conceptual layout and the results of the field calculation. A possible issue with this magnet is the mechanical stability of the coil.

At the second proton low beta quadrupole which needs the full horizontal aperture, the two beams are separated by $85 \mathrm{~mm}$ so that the lepton beam pipe can pass outside the regular coil through the flux return yoke. This magnet needs a wide flux return. The coil is a standard superconducting quadrupole coil with an aperture of $30 \mathrm{~mm}$. 


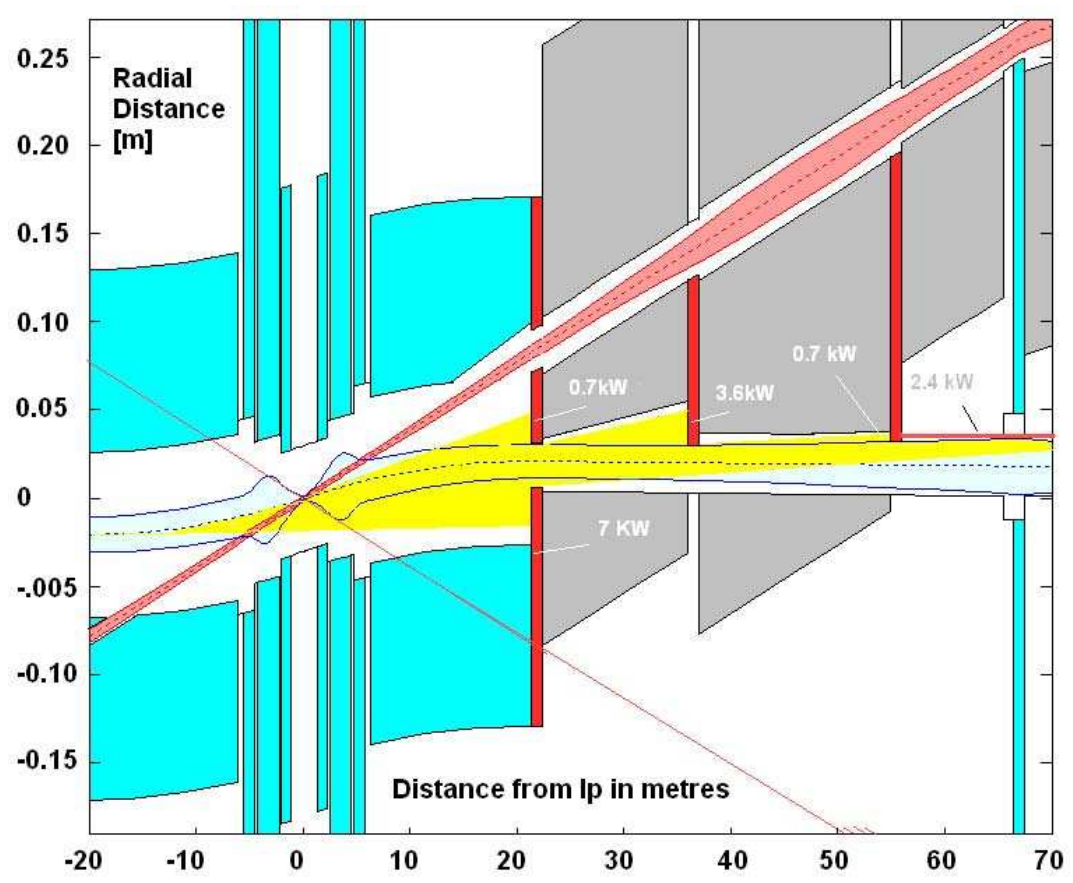

Figure 26: Deposition of Synchrotron radiation: Most of the power of $9.1 \mathrm{~kW}$ in the synchrotron radiation fan (shown in yellow) is absorbed at the collimator-absorber at $22 \mathrm{~m}$ from the IP (dark/red). No direct radiation is deposited inside the superconducting magnets.

\subsection{Beam-Beam Effects and Luminosity}

In the following, the luminosity will be recalculated from the design parameters. The large bunch length of the proton beam of $7.5 \mathrm{~cm}$ will cause a luminosity reduction since it enhances the effective beam size of the protons experienced by the electron beam. The effect of the short lepton bunch length can be neglected and the hour glass luminosity reduction factor is

$$
R=\frac{2 \cdot \beta_{y p} \beta_{y e}}{\sqrt{\pi} \sigma_{p} \sqrt{\beta_{y p}^{2}+\beta_{y e}^{2}}} \cdot \exp \left(2 \cdot \frac{\beta_{y p} \beta_{y e}}{\sigma_{p} \sqrt{\beta_{y p}^{2}+\beta_{y e}^{2}}}\right) \cdot K_{0}\left(\left(\frac{\beta_{y p} \beta_{y e}}{\sigma_{p} \sqrt{\beta_{y p}^{2}+\beta_{y e}^{2}}}\right)^{2}\right)=0.95
$$

The crossing angle should not reduce the luminosity if it is properly compensated by the crab-tilt of the proton bunches. Another effect which influences the luminosity is the so-called dynamic beta, the distortions of the beta functions in the core of the beam by the beam-beam interaction. This distortion decreases the lepton beta functions at the IP in the electron-proton case if the tunes are above the integer and below the quarter integer resonance

$$
\frac{\Delta \beta_{x, y}}{\beta_{x, y}}=-\frac{r_{0} \cdot N_{p} \cdot \beta_{x, y} \cdot \cot \left(2 \cdot \pi \cdot Q_{x, y}\right)}{\gamma_{e} \cdot \sigma_{x, y}^{p} \cdot\left(\sigma_{x}^{p}+\sigma_{y}^{p}\right)} .
$$



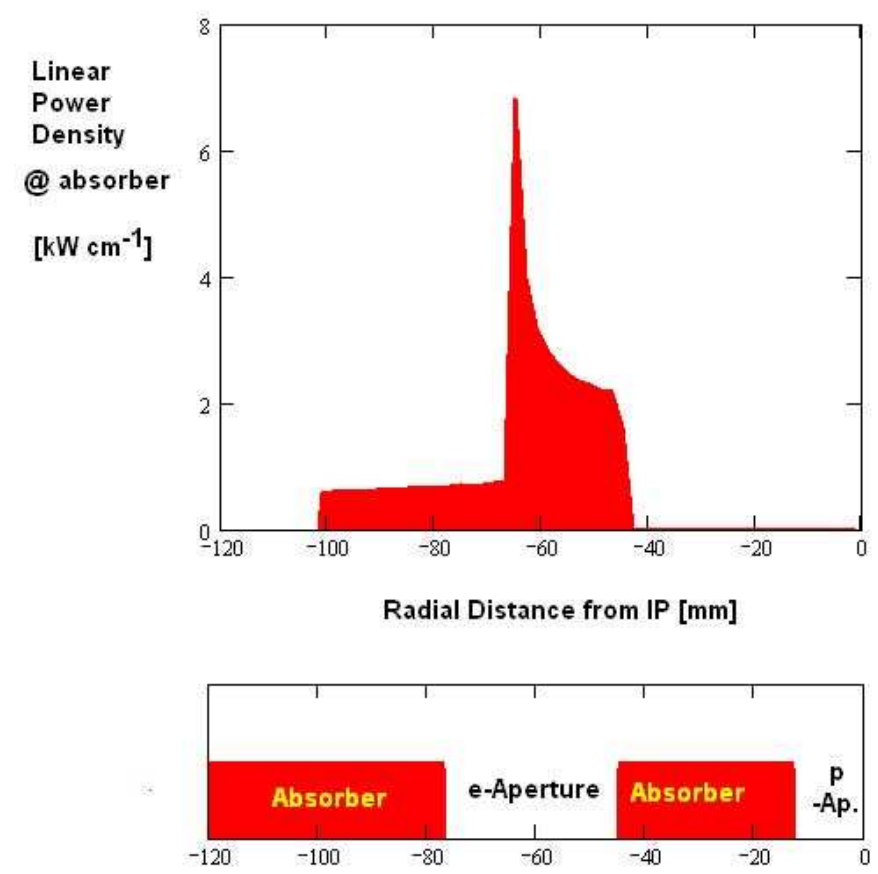

Figure 27: Synchrotron radiation linear power density at $21 \mathrm{~m}$ from the IP, at the entrance of the absorber. The red boxes below indicate the position of the absorber elements.

This does not affect the beam tails and has no influence on the aperture requirements for the beam. It does affect, however, the beam matching and the chromaticity. Dynamic focusing can therefore not be used to increase the luminosity without taking into account the considerations chromaticity limitations as discussed before. The dynamic beta depends strongly on the choice of the tunes. For very strong beam beam forces, the beta functions might be increased by higher order effects. For HERAe-like tunes $\mathrm{Q}_{x e}=0.10$ and $\mathrm{Q}_{y e}=0.11$ one obtains a strong reduction of the horizontal electron beta functions from $\beta_{x e}=12.7 \mathrm{~cm}$ to $\beta_{x e}=6.9 \mathrm{~cm}$, and a small increase for the vertical beta function from $\beta_{y e}=7 \mathrm{~cm}$ to $\beta_{y e}=7.3 \mathrm{~cm}$. Note that for positron rather than electron running, the tunes should be taken below the integer resonance.

The beam-beam tune shift values which result from the parameters are

$$
\begin{gathered}
\Delta \nu_{x}^{p}=\frac{r_{p} N_{e} \beta_{x p}}{2 \pi \gamma_{p} \sigma_{x e}\left(\sigma_{x e}+\sigma_{y e}\right)}=0.83 \cdot 10^{-3} \\
\Delta \nu_{y}^{p}=\frac{r_{p} N_{e} \beta_{y p}}{2 \pi \gamma_{p} \sigma_{y e}\left(\sigma_{x e}+\sigma_{y e}\right)}=0.32 \cdot 10^{-3} \\
\Delta \nu_{x}^{e}=\frac{r_{0} N_{p} \beta_{y e}}{2 \pi \gamma_{e} \sigma_{x p}\left(\sigma_{x p}+\sigma_{y p}\right)}=48 \cdot 10^{-3} \\
\Delta \nu_{y}^{e}=\frac{r_{0} N_{p} \beta_{y e}}{2 \pi \gamma_{e} \sigma_{y p}\left(\sigma_{x p}+\sigma_{y p}\right)}=51 \cdot 10^{-3} .
\end{gathered}
$$




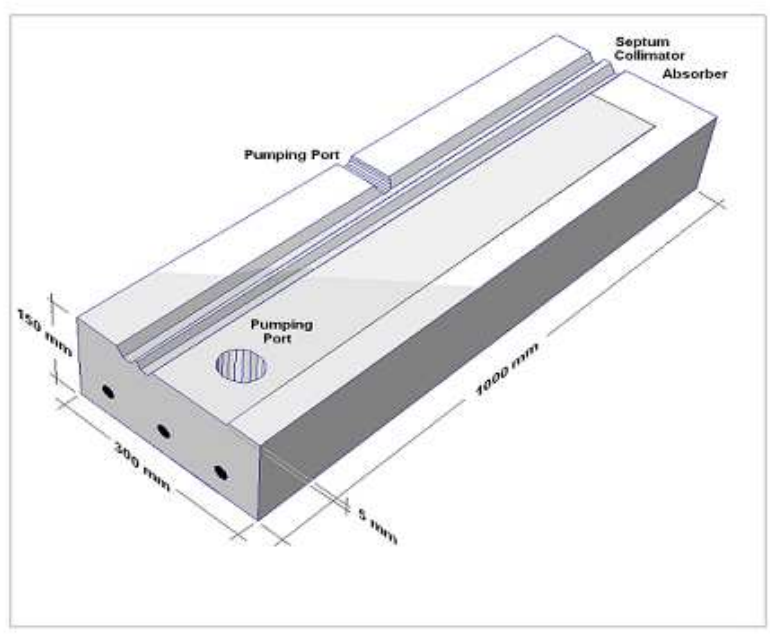

Figure 28: Sketch of the HERA-type, conical, $100 \mathrm{~cm}$ long, $30 \mathrm{~cm} \times 30 \mathrm{~cm}$ cross section, water-cooled synchrotron radiation absorber made of copper. The radiation is absorbed over a length of $400 \mathrm{~mm}$ and the full width of $90 \mathrm{~mm}$ of the absorber (only the lower half of the absorber is shown).

The vertical tune shift value is within the range of HERA operational parameters. However, large tune shifts in the order of 0.05 in both planes are beyond HERA experience. Given the larger beam energy of $70 \mathrm{GeV}$ and the shorter damping time, these tune shifts may be considered tolerable. More studies are needed to verify whether this choice is acceptable or whether the design should be based on a larger crossing angle, larger electron emittance and smaller beta.

The long-range beam-beam tune shift parameters $\Delta \nu_{x}^{p a r}, \Delta \nu_{y}^{p a r}$ of the lepton beam at the parasitic crossing points are given by [100]

$$
\begin{array}{r}
\Delta \nu_{x}^{p a r}=\frac{N_{p} r_{0} \beta_{x}^{p a r}}{2 \pi \gamma_{e} \Delta x^{2}} \\
\Delta \nu_{y}^{p a r}=\frac{-N_{p} r_{0} \beta_{y}^{p a r}}{2 \pi \gamma_{e} \Delta x^{2}}
\end{array}
$$

where $\beta_{x, y}^{\text {par }}$ are the beta functions, $\Delta x$ is the horizontal beam separation at the parasitic crossings and the vertical beam separation is zero. The corresponding values for the first parasitic crossing is quite large for a bunch spacing of 25ns (see Tab. 6 which summarizes the evaluation of these formulae). The operational experience with long-range beambeam effects in LEP [101] (at four instead of at one interaction point) indicates, that one might have to expect problems mainly due to beam-beam orbit effects. For larger bunch spacing of $50 \mathrm{~ns}$ and $75 \mathrm{~ns}$ the long range beam-beam parameters are sufficiently small. A somewhat larger crossing angle might be necessary relax the long-range beambeam effects sufficiently. Further study is needed to confirm whether a crossing angle of $2 \mathrm{mr}$ is sufficient for $25 \mathrm{~ns}$ bunch spacing.

Taking these effects into account the luminosity for $I_{e}=71 \mathrm{~mA}, N_{p}=1.6810^{11}, \epsilon_{p}=$ 


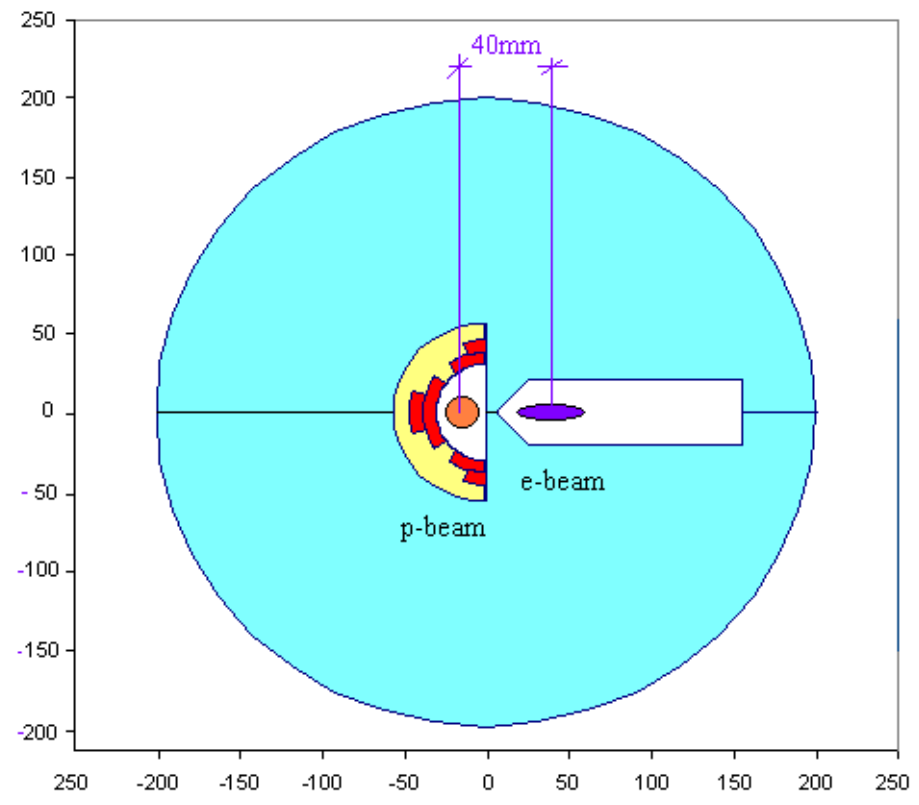

Figure 29: Sketch of the cross section of the $30 \mathrm{~mm}$ aperture septum quadrupole used for the vertical low beta lens of the proton beam.

$0.5 \mathrm{~nm}, \epsilon_{x e}=7 \mathrm{~nm}, \epsilon_{y e}=5 \mathrm{~nm}$ and $R=0.94$ amounts to

$$
L=\frac{I_{e} \cdot N_{p} \cdot R}{2 \cdot \pi \cdot e \cdot \sqrt{\epsilon_{p} \beta_{x p}+\epsilon_{x e} \beta_{x e}} \cdot \sqrt{\epsilon_{p} \beta_{y p}+\epsilon_{y e} \beta_{y e}}}=1.11 \cdot 10^{33} \mathrm{~cm}^{-2} s^{-1},
$$

according to the design goal.

\section{Summary}

The physics and the design of a Large Hadron Electron Collider (LHeC) are sketched. It is illustrated that a unique and important programme of physics is possible with a $70 \mathrm{GeV}$ electron/positron beam in collision with the $7 \mathrm{TeV}$ LHC proton (and ion) beam at a luminosity of $10^{33} \mathrm{~cm}^{-2} \mathrm{~s}^{-1}$.

Experiments at such a collider probe electron-quark and positron-quark physics in an unparalleled manner, thereby enabling a substantial extension of the discovery potential at the LHC and making possible measurements of a precision characteristic of lepton-hadron measurements. Highlights include

- Observation and precision measurement of new physics in the lepton-quark spectrum at the TeV scale, which could reveal unexpected and new leptoquark phenomena. The LHeC will provide precision measurements which are important to the interpretation and quantification of this new physics. 


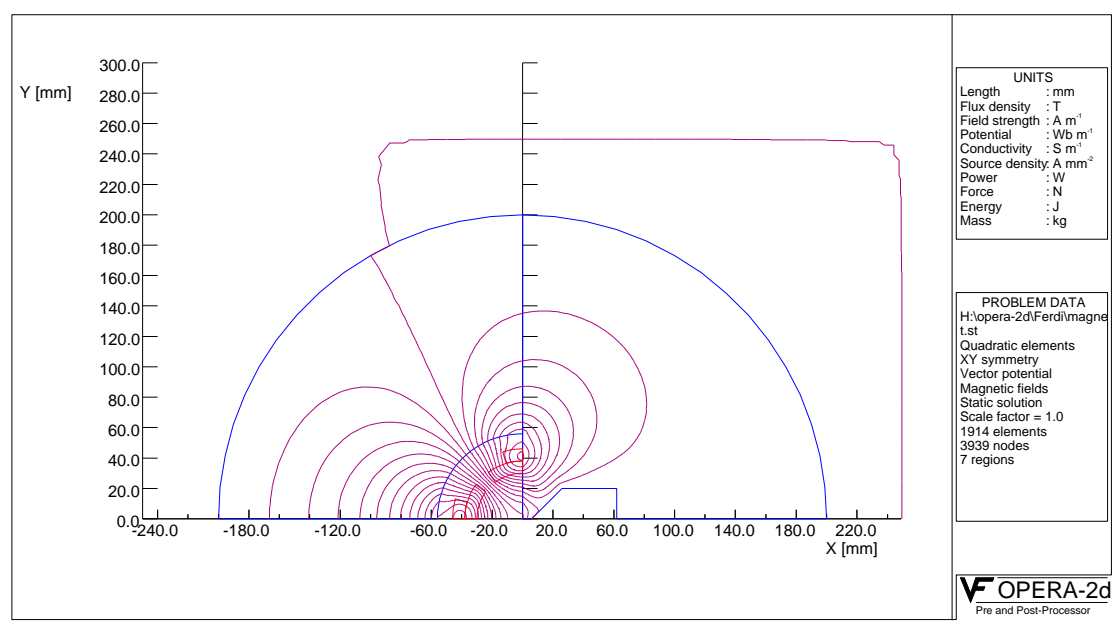

Figure 30: Field lines of the septum quadrupoles as calculated with OPERA-2D [22].

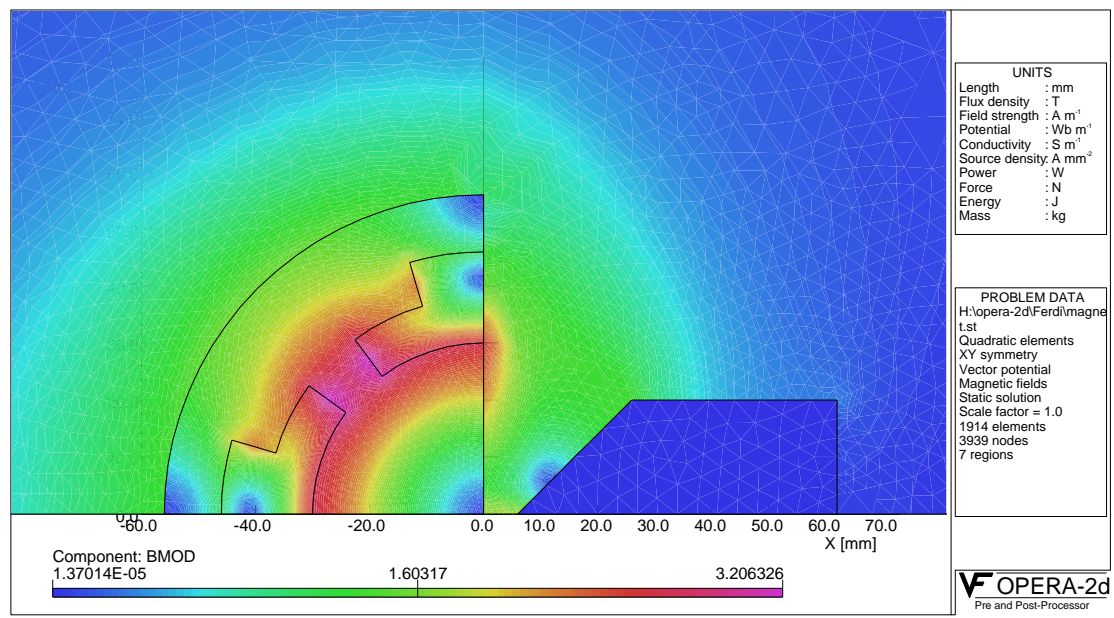

Figure 31: Field map of the septum quadrupole obtained with OPERA-2D [15]. The magnetic induction does not exceed values of $1.6 \mathrm{~T}$ and the mirror plate functions well for this magnet. The magnetic field inside the septum gap is only a few Gauss.

- Discovery and precision measurement of new physics in proton structure at very low Bjorken- $x$, which will be crucial to superhigh energy neutrino physics, to forward physics at the LHC and basically to the development of our understanding of QCD in the high parton density, low coupling limit, and thereby the phase equilibria of chromodynamics in a variety of hadronic systems at the TeV energy scale.

- A new level of precision measurements and precision tests of the validity of QCD at a new distance scale, corresponding to substructure dimensions of $10^{-19} \mathrm{~m}$, which promise to have a direct bearing on the overall consistency of the Standard Model and its underlying physics as one moves towards the unification scale.

- Measurements which will make possible the determination of parton distribution functions of nucleons and nuclei over a hitherto inaccessible kinematic range in 
Table 6: Parasitic beam-beam tune shifts of the lepton beam

\begin{tabular}{|l|l|l|l|l|l|}
\hline $\begin{array}{l}\text { Bunch } \\
\text { spacing } \\
\text { angle }\end{array}$ & $\begin{array}{l}\text { Crossing } \\
\text { Separation }\end{array}$ & Separation & $\begin{array}{l}\text { Horizont. } \\
\text { parasitic } \\
\text { beam-beam } \\
\text { tune shift }\end{array}$ & $\begin{array}{l}\text { Vertical } \\
\text { parasitic } \\
\text { beam-beam } \\
\text { tune shift }\end{array}$ \\
\hline$[\mathrm{ns}]$ & {$[\mathrm{mrad}]$} & {$[\mathrm{mm}]$} & {$\left[\sigma_{e x}\right]$} & & \\
\hline 25 & 2.0 & 7.7 & 8.3 & 0.0011 & -0.0015 \\
\hline 50 & 2.0 & 15 & 23 & 0.0001 & -0.002 \\
\hline 75 & 2.0 & 27 & 50 & 0.00003 & -0.0004 \\
\hline
\end{tabular}

probe scale $\left(Q^{2}\right)$ and longitudinal momentum fraction (Bjorken- $x$ ), and which are essential if the sensitivity at the LHC to new and rare physics in both $p p$ and $e p$ physics is to be optimised.

- The immense energy densities achieved in an $A A$ interaction at the LHC. To fully explore the nature of the interactions will require comparable data in $p A$, $p p$, and $e A$ collisions. LHeC and the LHC will thus constitute an experimental tool unparalleled in the history of hadron physics in that nowhere else has there ever been such a range of possible measurements at such an energy scale.

A conceptual design of a high luminosity Large Hadron Electron Collider, the LHeC, is presented. The approach takes advantage of developments in technology which are now well advanced, and which are necessary for future electron/positron linear accelerators, to achieve an electron/positron storage ring of for example $70 \mathrm{GeV}$ energy in the LHC tunnel. It is shown how, with the careful design of the RF structure and the interaction region, it is possible to achieve a luminosity of $10^{33} \mathrm{~cm}^{-2} \mathrm{~s}^{-1}$ in collisions with one of the LHC hadron beams. The solution is based on $25 \mathrm{~ns}$ bunch spacing with a small crossing angle of $2.0 \mathrm{mrad}$ which requires crab-crossing for the proton beam. The concept is aimed at the simultaneous operation of LHC and LHeC and first considerations of how this can be achieved are stated. Wherever possible, realistic constraints are included based on past operation of electron storage rings and on the operation of the HERA electron and proton storage rings at DESY Hamburg.

Further work is needed to address a number of issues which have yet to be resolved concerning the feasibility of such a concept. Nevertheless, to date it appears not unreasonable to continue to contemplate a major and important ep physics programme at the $\mathrm{TeV}$ scale as part of the physics programme of the LHC. 


\section{Acknowledgement}

The authors wish to thank G. Altarelli, W. Bartel, J. Blümlein, S. Brodsky, A. Bruell, A. Caldwell, A. DeRoeck, M. Diehl, J. Ellis, R. Engel, J. Engelen, J. Jowett, H. Jung, J. Feltesse, S. Forte, A. Glazov, T. Greenshaw, R. Heuer, E. Kabuss, U. Katz, H. Kowalski, T. Laštovička, A. Levy, M. Mangano, M. Marx, S. Myers, C. Niebuhr, D. Pitzl, B. Reisert, R. Rueckl, D. Schlatter, S. Schlenstedt, M. Seidel, R. Schmitt, U. Stoesslein, M. Strikman, R.E. Taylor, W. K. Tung, R. Valbuena, and A. Zarnecki for interesting discussions and providing valuable information.

The authors have worked for two decades at the world's first high energy collider of different particle species, HERA at DESY. It would be inappropriate not to record our thanks also to many colleagues, too numerous to name here, with whom we have worked throughout this time at HERA and who have developed the physics and the techniques for experimentation at such a collider on which much of what is sketched in this paper relies.

\section{References}

[1] R. P. Feynman, Lepton Hadron Interactions, New York 1972.

[2] R. P. Feynman, Phys. Rev. Lett. 23 (1969) 1415;

J. D. Bjorken, Phys. Rev. D1 (1970) 1376;

J. D. Bjorken and E. A. Paschos, Phys. Rev. 185 (1969) 1975.

[3] J. I. Friedman, H. W. Kendall, R. E. Taylor, Nobel Prize lectures 1990.

[4] D. Gross, H. Politzer, F. Wilczek, Nobel Prize lectures 2004.

[5] R. Brinkmann, Snowmass Workshop 2001, Proceedings;

F. Willeke, Snowmass Workshop 2001, Proceedings;

see also the THERA Book [9].

[6] D. Schulte and F.Zimmermann, "QCD Explorer based on LHC and CLIC-1", CERN AB-2004-079, CLIC Note 608;

see also L. Gladilin et al., hep-ph/0504008.

[7] J. Feltesse, Talk at the LHC Workshop, Aachen 1990, CERN 90-10 (1990), Proceedings Vol.1 p.219, eds. C. Jarlskog and D. Rein.

[8] R. Rückl, Talk at the LHC Workshop, Aachen 1990, CERN 90-10 (1990), Proceedings Vol.1 p.229, eds. C. Jarlskog and D. Rein.

[9] The THERA Book, ep Scattering at $\sqrt{s} \sim 1$ TeV, DESY 01-123F, eds. U. Katz, M. Klein, A. Levy and S. Schlenstedt.

[10] A. Verdier, Talk at the LHC Workshop, Aachen 1990, CERN 90-10 (1990), Proceedings Vol.1 p.820, eds. C. Jarlskog and D. Rein. 
[11] W. Bartel, ibid, p.824.

[12] E. Keil, LHC Project Report, 93(1997).

[13] CERN Council Strategy Group, http://council-strategygroup.web.cern.ch/council-strategygroup/

[14] A. Zarnecki in [9] and private communication.

[15] A. Belyaev, C. Leroy, R. Mehdiyev and A. Pukhov, JHEP 0509 (2005) 005 [hep-ph/0502067].

[16] J. Ohnemus, S. Rudaz, T. F. Walsh and P. M. Zerwas, Phys. Lett. B 334 (1994) 203 hep-ph/9406235.

[17] P. Taxil, E. Tugcu and J.M. Virey, Eur. Phys. J. C14 (2000) 165 hep-ph/9912272.

[18] A. Djouadi et al., Z. Phys. C46 (1990) 679.

[19] W. Buchmüller, R. Rückl and D. Wyler, Phys. Lett. B191 (1987) 442. Erratum Phys. Lett. B448 (1999) 320.

[20] I. Abt et al. H1 Collaboration, Nucl. Phys. B 407 (1993) 515;

M. Derrick et al. ZEUS Collaboration, Phys. Lett. B 316 (1993) 412;

C. Adloff et al. H1 Collaboration, Phys. Lett. B 520 (2001) 183;

J. Breitweg et al. ZEUS Collaboration, Eur. Phys. J. C 7 (1999) 609.

[21] F. Wilczek, Int. J. Mod. Phys. A 16 (2001) 1653 hep-ph/0101187.

[22] H1 Collaboration, C. Adloff et al., Eur. Phys. J. C21 (2001) 33 hep-ex/0012053|.

[23] ZEUS Collaboration, S. Chekanov et al., Eur. Phys. J. C 42 (2005) 1 hep-ph/0503274.

[24] V.N. Gribov, E.M. Levin and M.G. Ryskin, Phys. Rep. 100 (1983) 1.

A. Mueller, hep-ph/0501012 and references therein.

[25] E. Iancu, A. Leonidov and L. McLerran, Nucl. Phys. A692 (2001) 583;

For a review and references see: E. Iancu and M. Venugopalan, Review for QGP3, Eds. R. C. Hwa and X.-N. Wang, World Scientific hep-ph/0303204.

[26] E. A. Kuraev, L. N. Lipatov and V. S. Fadin, Sov. Phys. JETP 44 (1976) 443 [Zh. Eksp. Teor. Fiz. 71 (1976) 840];

E. A. Kuraev, L. N. Lipatov and V. S. Fadin, Sov. Phys. JETP 45 (1977) 199 [Zh. Eksp. Teor. Fiz. 72 (1977) 377];

I. I. Balitsky and L. N. Lipatov, Sov. J. Nucl. Phys. 28 (1978) 822 [Yad. Fiz. 28 (1978) 1597]. 
[27] M. Ciafaloni, Nucl. Phys. B 296 (1988) 49;

S. Catani, F. Fiorani and G. Marchesini, Phys. Lett. B 234 (1990) 339;

S. Catani, F. Fiorani and G. Marchesini, Nucl. Phys. B 336 (1990) 18;

G. Marchesini, Nucl. Phys. B 445 (1995) 49.

[28] I. Balitsky, Nucl. Phys. B463 (1996) 99 hep-ph/0101042;

Yu. V. Kovchegov, Phys. Rev. D60 (1999) 034008; ibid. D61 (2000) 074018.

[29] V. N. Gribov and L. N. Lipatov, Sov. J. Nucl. Phys. 15 (1972) 675 [Yad. Fiz. 15 (1972) 1218];

V. N. Gribov and L. N. Lipatov, Sov. J. Nucl. Phys. 15 (1972) 438 [Yad. Fiz. 15 (1972) 781];

L. N. Lipatov, Sov. J. Nucl. Phys. 20 (1975) 94 [Yad. Fiz. 20 (1974) 181];

G. Altarelli and G. Parisi, Nucl. Phys. B 126 (1977) 298;

Y. L. Dokshitzer, Sov. Phys. JETP 46 (1977) 641 [Zh. Eksp. Teor. Fiz. 73 (1977) 1216].

[30] G. Altarelli, R.D. Ball and S. Forte, hep-ph/0512237.

[31] S. Moch, J. Vermaseren and A. Vogt, Nucl. Phys. 688 (2004) 101 and 691 (2004) 129 and Nucl. Phys. B 724 (2005) 3 hep-ph/0504242].

[32] A.H. Müller, Nucl. Phys. B415 (1994) 373; ibid. B437 (1995) 107;

A.H. Müller and B. Patel, Nucl. Phys. B425 (1994) 471.

[33] K. Golec-Biernat, M. Wüsthoff, Phys. Rev. D59 (1999) 014017;

K. Golec-Biernat, M. Wüsthoff, Phys. Rev. D59 (1999) 114023;

J. Bartels, K. Golec-Biernat, H. Kowalski, Phys. Rev. D66 (2002) 014001.

[34] J. Bartels, Eur. Phys. J. C 43 (2005) 3.

[35] E. Gotsman, E. Levin, U. Maor, E. Naftali, hep-ph/0504040.

[36] H1 Collab., A. Aktas et al., Accepted by Eur Phys J, hep-ex/0508055;

ZEUS Collab., S. Chekanov et al., Phys. Lett. B632 (2006) 13 [hep-ex/0502029].

[37] H1 Collab., A. Aktas et al., Eur. Phys. J. C33 (2004) 477 [hep-ex/0310019].

[38] ALICE Collab., F Carminati et al., J. Phys. G: Nucl. Part. Phys. 30 (2004) 1517.

[39] V. Gribov, Sov. Phys. JETP 30 (1970) 709.

[40] For a review and references see: R. Engel, astro-ph/0504358.

[41] S. Brodsky et al., Phys.Rev. D65 (2002) 114025.

[42] M. Klein and T. Riemann, Z. Phys. C 24 (1984) 151.

[43] D. Mason, NuTeV Collaboration, hep-ex/0405037.

S. Kretzer and F. I. Olness, AIP Conf. Proc. 792 (2005) 843 hep-ph/0508216. 
[44] M. Dittmar, F. Pauss and D. Zurcher, Phys. Rev. D56 (1997) 7284 [hep-ex/9705004]; A. Cooper-Sarkar et al., Summary of Structure Function Working Group [48].

[45] W.J. Stirling, Talk at the Binn Workshop on Physics at the LHC, October 2003.

[46] A. D. Martin, R. G. Roberts, W. J. Stirling and R. S. Thorne, Eur. Phys. J. C 35 (2004) 325 arXiv:hep-ph/0308087.

A. Cooper-Sarkar et al., Summary of Structure Function Working Group [48].

[47] A. Martin, M. Ryskin, G. Watt, Eur.Phys.J. C37 (2004) 285.

[48] Proceedings of the "HERA and the LHC Workshop", CERN-2005-014, DESYPROC-2005-001, Eds. A. De Roeck and H. Jung.

[49] H1 Collab., A. Aktas et al., Eur. Phys. J. C40 (2005) 349 [hep-ex/0411046];

H1 Collab., A. Aktas et al., Eur. Phys. J. C45 (2006) 23 hep-ex/0507081.

[50] B.C. Allanach et al., hep-ph/0403133.

[51] For a recent calculation see: Q. Mason et al, hep-lat/0503005.

[52] W. van Neerven, in [9].

[53] C. Glasman, hep-ex/0506035.

[54] Wu Ki Tung, private communication.

[55] A. D. Martin, R. G. Roberts, W. J. Stirling and R. S. Thorne, hep-ph/0307262.

[56] V. Khoze, A. Martin, M. Ryskin, Eur. Phys. J C23 (2002), 311;

A. de Roeck et al., Eur. Phys. J C25 (2002) 391.

[57] ZEUS Collaboration, M. Derrick et al., Phys. Lett. B315 (1993) 481;

H1 Collaboration, T. Ahmed et al., Nucl. Phys. B429 (1994) 477.

[58] J. Collins, Phys. Rev. D57 (1998) 3051 and erratum-ibid. D61 (2000) 019902.

[59] See for example H1 Collaboration, C. Adloff et al., Zeit. Phys. C76 (1997) 613.

[60] For a review see J. Collins et al., in 48].

[61] M. Krawczyk and M. Zembrzuski in [9].

[62] D. Mueller et al., Fortschritte der Physik 42 (1994) 101 hep-ph/9812448];

For a review see M. Diehl, Phys. Rep. 388 (2003) 41.

[63] For a review see A.V. Belitsky and A.V. Radyushkin, hep-ph/0504030.

[64] A.V. Belitsky and D. Müller, hep-ph/0206306.

[65] S. V. Goloskokov and P. Kroll, Eur. Phys. J. C 42 (2005) 281 hep-ph/0501242. 
[66] M. Arneodo et al., hep-ph/9610423.

[67] T. Alexopoulos et al., eD Scattering with H1, A Letter of Intent, DESY 03-194; H. Abramowicz et al., A New Experiment for HERA, MPP-2003-62;

F. Willeke and G. Hoffstaetter, Talks at the Workshop on the Future of DIS, Durham 2001, unpublished;

M. Klein and T. Greenshaw, J. Phys. G Nucl. Part. Phys. 28 (2002) 2505; http://hep.ph.liv.ac.uk/ green/HERA3/.

[68] L. Merminga and Yu. Derbenev, Contribution to CIPANP2003;

R. Ent, Report to NSAC Subcommittee, BNL, June 2004, http://nsac2004.bnl.gov/pres/ent.pdf.

"Electron-Ion Collider at CEBAF: New Insights and Conceptual Progress", Y. Derbenev, et al., Proc. EPAC 2004

[69] eRHIC homepage, http://www.phenix.bnl.gov/WWW/publish/abhay/

Zero-design-Report http://www.rhichome.bnl.gov/eRHIC/

[70] R. Milner, Report to NSAC Subcommittee, BNL, June 2004, eRHIC [69].

[71] For reviews and references see: M. Gyulassy and L. Mc Lerran, Nucl. Phys. A 750 (2005) 30 nucl-th/0405013;

A. Mueller and A.I. Soshi, hep-ph/0402193.

[72] L. Frankfurt and M. Strikman, Eur. Phys. J. A5(1999)293.

[73] M. Strikman, private communication.

[74] L. Frankfurt, V. Guzey, M. McDermott and M. Strikman, hep-ph/0104154.

see also L. Frankfurt, M. Strikman and C. Weiss, Ann. Rev. Nucl. Sci. 55 (2005) 403.

[75] S. Forte, private communication.

[76] M. Botje, M. Klein and C. Pascaud, HERA Workshop 1996, Proceedings Hamburg 1996, p. 33, eds. G. Ingelman, R. Klanner and A. De Roeck hep-ph/9609489].

[77] A. Blondel and F. Jacquet, Proceedings of the Study of an ep Facility for Europe, ed. U. Amaldi, DESY (1979) 391.

[78] TOTEM Collaboration, V. Beradi et al., technical design report, CERN-LHCC2004-002

[79] http://www.fp420.com/index.html;

FP420 : An R+D Proposal to Investigate the Feasibility of Installing Proton Tagging Detectors in the $420 \mathrm{~m}$ Region at LHC, M.G. Albrow et al., CERN-LHCC-2005-025;

[80] M. Tigner, B. Wiik and F. Willeke, An electron proton collider in the TeV range, in Proc. IEEE Particle Accelerator Conference, San Franscisco, p.2910 (1991). 
[81] www.SLAP.web.cern.ch/slap/lhc-parameter.htm

[82] R. Brinkmann, F. Willeke: FIRST EXPERIENCE WITH COLLIDING ELECTRON PROTON BEAMS IN HERA, Prepared for 1993 IEEE Particle Accelerator Conference (PAC 93), Washington, DC, 17-20 May 1993. Particle accelerator, vol. $5^{*} 3742-3744$.

[83] S. Poulsen, CERN, Geneva, Switzerland: ?AN INDUSTRIAL CONTROL SYSTEM FOR THE SUPERVISION OF THE CERN ELECTRICAL DISTRIBUTION NETWORK?, International Conference on Accelerator and Large Experimental Physics Control Systems, 1999, Trieste, Italy; http://cernenviro.web.cern.ch/CERNenviro/Pages/E/Themes/Electricity/Electricity.html

[84] M. Sands, The Physics of Electron Storage Rings, Lecture given at the Ravenna School for Accelerator Physics 1976, unpublished

[85] R. Brinkmann, F. Willeke, CHROMATIC CORRECTIONS AND DYNAMIC APERTURE IN THE HERA ELECTRON RING. 2. DESY 87/037, May 1987;

R. Brinkmann, F. Willeke: CHROMATIC CORRECTIONS AND DYNAMIC APERTURE IN THE HERA ELECTRON RING, in Novosibirsk 1986, Proceedings, High energy accelerators, vol. 1* 87-90 and Hamburg DESY - DESY M-86-10 (86.) 5-8.

[86] F. Willeke, Proceedings of the 2nd Advanced ICFA Beam Dynamics Workshop, Lugano,1988, p.164, CERN 88-04 (1988).

[87] G. Hoffstaetter, F. Willeke: ELECTRON DYNAMICS IN THE HERA LUMINOSITY UPGRADE LATTICE OF THE YEAR 2000, DESY-M-99-02U, Jun 1999. 3pp., prepared for IEEE Particle Accelerator Conference (PAC 99), New York, NY, 29 Mar - 2 Apr 1999, published in *New York 1999, Particle Accelerator, vol. 1* 407-409

[88] A. Verdier, Proceedings of the 2nd Advanced ICFA Beam Dynamics Workshop, Lugano, 1988, p.186ff, CERN 88-04 (1988)

[89] The LEP Design Report, Vol II, CERN-LEP, 84-01 (1984)

[90] M. Bieler et al., RECENT AND PAST EXPERIENCES WITH BEAM-BEAM EFFECTS AT HERA, SLAC-PUB-8203, Nov 1999, prepared for Workshop on Beam-Beam Effects in Large Hadron Colliders, Geneva, Switzerland, 12-16 Apr 1999 inGeneva 1999, Beam-beam effects in large hadron colliders* 12-19.

[91] M.G. Minty: HERA PERFORMANCE UPGRADE: ACHIEVEMENTS AND PLANS FOR THE FUTURE, EPAC-2004-TUXLH02, Jul 2004. 5pp, presented at the 9th European Particle Accelerator Conference (EPAC 2004), Lucerne, Switzerland, 5-9 Jul 2004

[92] O. Gröbner, Experience from the LEP Vacuum System, Proceedings of the Workshop an VLHC, ITT, (2001) 
[93] S. Belomestnykh, P. Barnes, E. Chojnacki, R. Ehrlich, R. Geng, D. Hartill, R. Kaplan, J. Knobloch, E. Nordberg, H. Padamsee, S. Peck, P. Quigley, J. Reilley, D. Rubin, J. Sears, V. Veshcherevich: COMMISSIONING OF THE SUPERCONDUCTING RF CAVITIES FOR THE CESR LUMINOSITY UPGRADE, Proceedings of the 1999 Particle Accelerator Conference, New York, 1999

[94] I.Ben-Zvi, Review of various approaches to address high currents in superconducting linacs, 12th International workshop on RF Superconductivity, Cornell July 2005 (2005)

[95] U. Schneekloth (ed), The HERA Luminosity Upgrade, DESY-HERA (1998)

[96] M. Seidel, HERA LUMINOSITY UPGRADE, DESY-M-99-02ZB, Jun 1999. 3pp. Prepared for IEEE Particle Accelerator Conference (PAC 99), New York, NY, 29 Mar - 2 Apr 1999, Published in *New York 1999, Particle Accelerator, vol. 1* 34.

[97] M. Bieler, E. Lohrmann, A. Meseck, G. Nawrath, M. Seidel, F. Willeke: DESIGN OF A HIGH POWER SYNCHROTRON RADIATION ABSORBER FOR HERA, DESY-M-99-02I, Jun 1999. 3pp. ,prepared for IEEE Particle Accelerator Conference (PAC 99), New York, NY, 29 Mar - 2 Apr 1999, Published in *New York 1999, Particle Accelerator, vol. ${ }^{*} 554$.

[98] M. Seidel, M. Hoffmann, VACUUM INDUCED BACKGROUNDS IN THE NEW HERA INTERACTION REGIONS, EPAC-2004-MOPLT045, DESY-M-04-03ZA, Oct 2004. 3pp. , Prepared for 9th European Particle Accelerator Conference (EPAC 2004), Lucerne, Switzerland, 5-9 Jul 2004.

[99] http://www.vectorfields.com/content/view/20/43/

[100] J. Jowett, in Handbook of Accelerator Physics and Engineering, A. Chao, M. Tigner, eds, World Scientific, Singapore (1999)

[101] W. Herr, EXPERIENCE WITH BEAM-BEAM EFFECTS, Nov 1999, prepared for Workshop on Beam-Beam Effects in Large Hadron Colliders, Geneva, Switzerland, April 1999, Beam-beam effects in large hadron colliders* 12-19. 